\title{
Acoustic Analysis of Spacecraft Cavities using the Boundary Element Method
}

\author{
Peter J. Marshall \\ Thesis submitted to the Faculty of the \\ Virginia Polytechnic Institute and State University \\ In partial fulfillment of the requirements for the degree of \\ Master of Science \\ in \\ Aerospace Engineering \\ Rakesh K. Kapania \\ Michael K. Philen \\ Thomas D. McQuigg
}

April 27, 2018

Blacksburg, Virginia

Keywords: Acoustics, Spacecraft Structures, Boundary Element Method Copyright $@ 2018$ by Orbital ATK 


\title{
Acoustic Analysis of Spacecraft Cavities using the Boundary Element Method
}

\author{
Peter J. Marshall \\ ABSTRACT
}

Spacecraft structures are subject to a series of load environments during their service life, with the most severe of these occurring during the spacecraft's launch and ascension through the atmosphere. In particular, acoustic loads imposed on stowed satellites within the launch vehicle fairing can result in high mechanical loads on sensitive spacecraft hardware. These acoustic loads have the potential to damage important components and as such it is necessary to accurately characterize and predict the acoustic launch environment for a given mission. This research investigates the Sound Pressure Level (SPL) that can be measured in and around spacecraft cavities resulting from a known excitation and the resultant structural responses. Linear finite element analysis (FEA) is coupled with the Boundary Element method (BEM) to analyze spacecraft acoustic environments and corresponding structural responses at low frequencies on the order of the structural modes.

Analytical capability for predicting acoustic environments inside the launch vehicle has improved significantly in recent years; however, while it is easy to perform an analysis and obtain results, the modeling effort can become unnecessarily complicated and analytical data can be hard to interpret. This work seeks to alleviate unnecessary complexity in the low-frequency regime of acoustic modeling by examining the fundamentals of coupled BEM-FEM analysis and applying simplification to a spacecraft model where possible to achieve results verified against direct field acoustic testing (DFAT) methods. 


\title{
Acoustic Analysis of Spacecraft Cavities using the Boundary Element Method
}

\author{
Peter J. Marshall \\ GENERAL AUDIENCE ABSTRACT
}

The modern spacecraft is a complicated assembly inclusive of panels, sophisticated instruments, harnesses, actuators, tanks, reflectors, and connecting hardware. Throughout its service life, it will be subjected to a series of dynamic load environments that have the potential to cause damage or compromise the intended mission. These environments are anticipated and simulated both analytically and experimentally to qualify the spacecraft within some confidence level.

One of the most severe dynamic environments that a spacecraft faces is the acoustic loading created by noise from the rocket engines at launch and aerodynamic turbulence on the launch vehicle during ascension. These noise levels, well above the threshold of human pain, cause the structure to vibrate at a variety of frequencies with significant force. Anticipated acoustic environments are simulated for spacecraft assemblies in testing using advanced audio equipment in efforts to produce equivalent measureable structural responses. In recent years, commercial software has been developed to create computer models of spacecraft that can be studied to predict these intense vibrations and where they will happen, which serves as an important consideration in the design process. Efforts are underway to improve the fidelity of these analytical models and correlate them with measured test data.

This work uses analytical models for the acoustic test environment at low frequencies to predict field levels between closely-spaced structural panels and the associated structural vibrations produced. Results are compared with test data and a trade study is conducted to assess modeling techniques and assumptions. 
Dedicated to my Lord and Savior Jesus Christ, whose power is made perfect in my weakness. 


\section{Acknowledgements}

First and foremost I would like to thank my advisor, Prof. Rakesh Kapania, for his consistent support and guidance over the past two years. When we first met, I had little research experience and it was difficult for me to articulate specific topics of interest outside of a desire to learn more about the structural design of flying things. Nevertheless, he believed in me, helped me explore a variety of research opportunities, and showed me the opportunity to work at Orbital ATK as an intern and gain valuable experience. I have had the privilege of taking two of his courses in structural dynamics and learning much from him and his other graduate students both inside and outside the classroom. I am grateful for his patience and wisdom in helping me achieve my goals.

I would also like to acknowledge the team at Orbital ATK: my committee member Dr. Thomas McQuigg, Mechanical Analysis Director Dr. Tom Stoumbos, Dr. Daisaku Inoyama, and Nguyen Do, among other engineers in the Mechanical Analysis group who supported me throughout the research process. My year-long internship at Orbital ATK was my first experience in the aerospace industry and the skills I learned in that work environment carried over directly to this endeavor. I thank Dr. Tom Stoumbos for bringing me into this space and helping me find my place in the company's ongoing research initiatives. Thank you to Nguyen Do, who has the heart of a teacher and served as a mentor for much of my internship, patiently answering my questions and explaining difficult concepts in ways that I could understand. I am thankful to Dr. Thomas McQuigg for helping me define my research objectives from the beginning and establish a framework for consistent progress and communicating my results. His expertise in acoustic analysis and testing has been tremendously helpful. Thank you also to Dr. Daisaku Inoyama for helping with the experimental setup for acquiring the test data presented in this work. Dr. Stoumbos, Dr. McQuigg, and Dr. Inoyama consistently took time out of their busy schedules to 
meet with me to review my progress and provide constructive feedback. Their support and encouragement has been invaluable.

This work is the fruit of an ongoing research collaboration between Orbital ATK and the Kevin T. Crofton Department of Aerospace and Ocean Engineering at Virginia Tech and it represents a concerted effort to bring graduate students into the aerospace industry workplace to apply what they have learned in the classroom to investigate significant engineering problems. It is my hope that the opportunities I have been blessed with at Orbital ATK continue to be extended to future graduate students pursuing a variety of research interests. 


\section{Table of Contents}

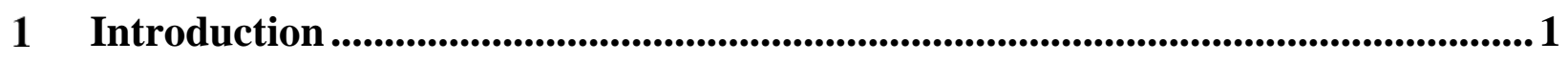

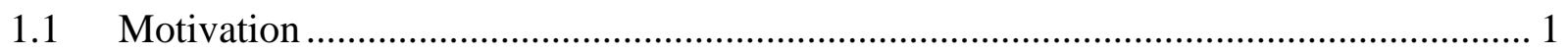

1.2 Spacecraft Acoustic Environments ............................................................................ 3

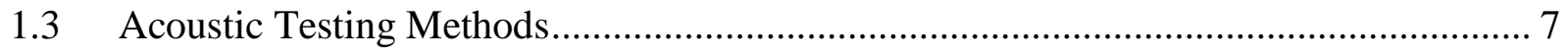

1.4 Acoustic Analysis Techniques ................................................................................ 8

\section{Acoustic Testing ...........................................................................................................10}

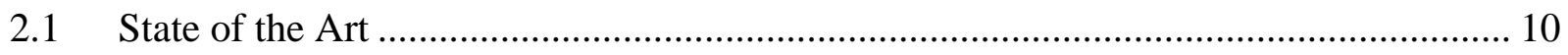

2.2 Direct Field Acoustic Testing (DFAT) .................................................................... 13

$2.3 \quad$ Test Procedure

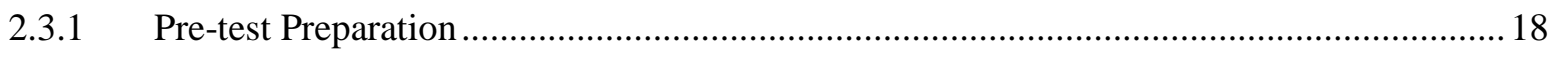

2.3.2 DFAT Test Setup Preparation ……………………………………………………. 19

2.3.3 Acoustic Field Shaping .........................................................................................2 20

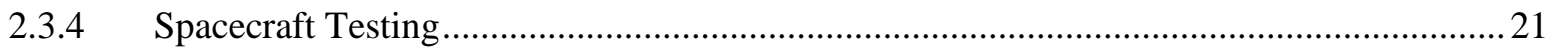

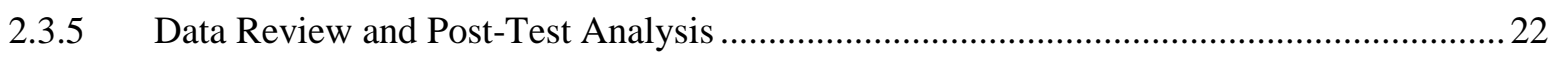

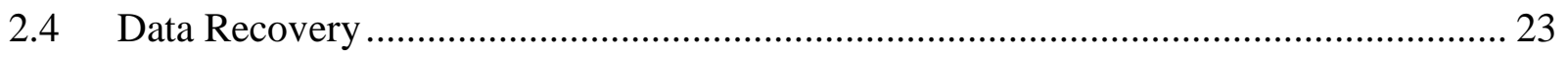

\section{Acoustic Analysis Using a Coupled FEM - BEM Approach ...................................26}

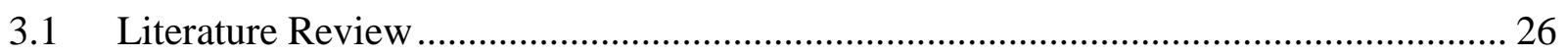

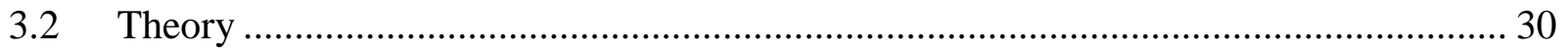

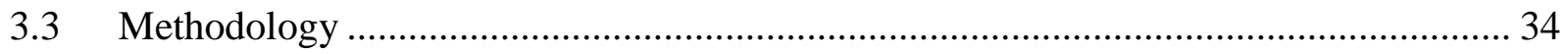

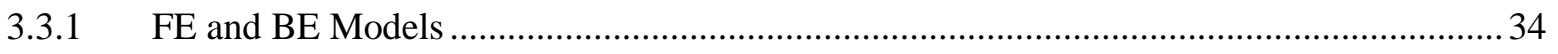

3.3.2 Boundary Conditions …………………………………………………………..... 40

3.3.3 Acoustic Excitation .............................................................................................. 40

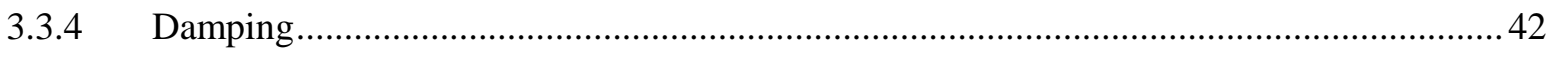

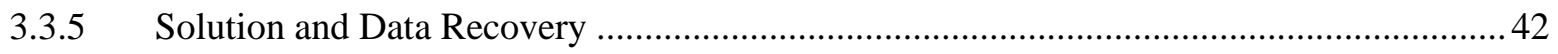




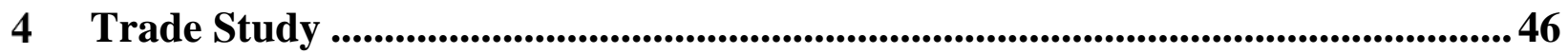

4.1 Definitions and Parameters of Interest ................................................................ 46

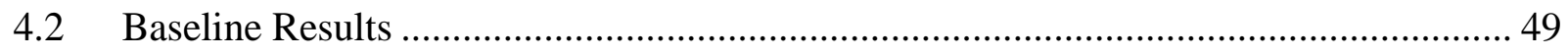

4.3 Solar Array Spacing Trades ................................................................................. 54

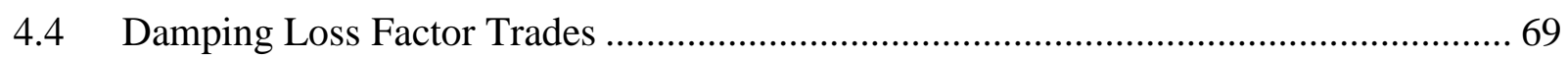

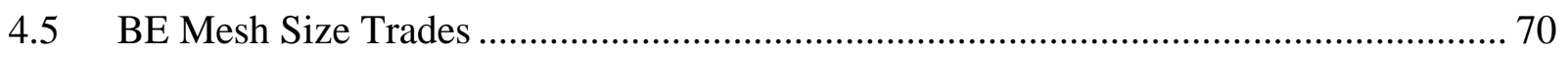

5 Conclusions ................................................................................................................ 74

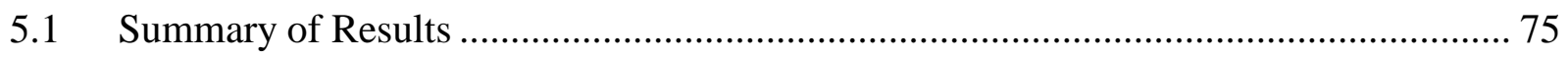

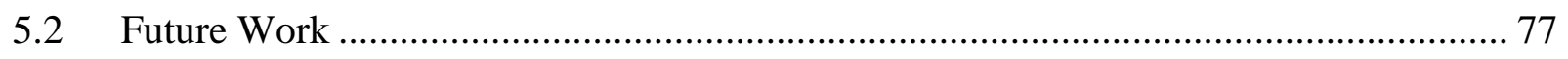

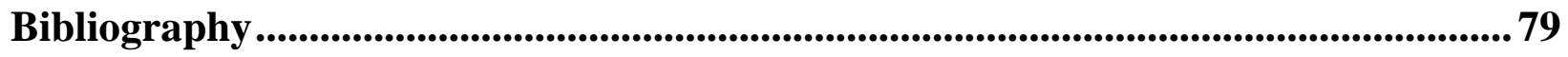

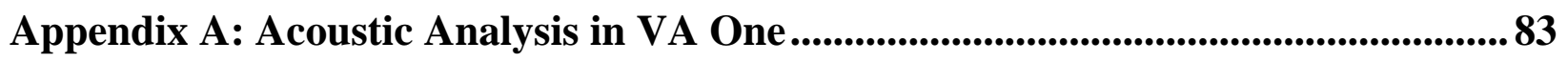

A.1 Coupled FEM-BEM Modeling Process .................................................................... 83

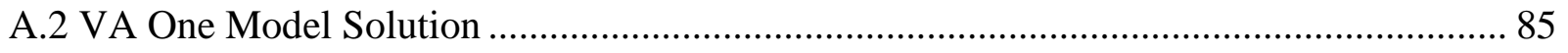

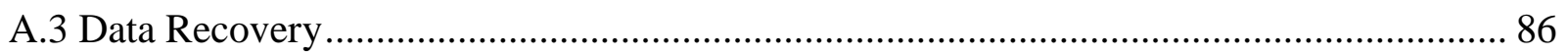




\section{List of Tables}

Table 2-1. Comparison of Acoustic Testing Methods ................................................................... 12

Table 3-1. Baseline Acoustic Model Solution Parameters ………………………………......... 43 


\section{List of Figures}

Figure 1-1. Overall sound pressure levels around rocket for flight and launch cases [3].............. 7

Figure 2-1. Diagram of Reverberant Chamber Test Configuration [1] ....................................... 11

Figure 2-2. DFAT Speaker Arrangement around Spacecraft [5]................................................ 13

Figure 2-3. Floor Plan for DFAT with Loudspeaker and Microphone Arrangement [5]............. 14

Figure 2-5. Predicted cylindrical cavity modes at (a) $88 \mathrm{~Hz}$ and (b) $237 \mathrm{~Hz}$ [7]....................... 16

Figure 2-6. Typical Launch Vehicle Fairing Acoustic Environments [11]-[13]......................... 19

Figure 2-7. Cutaway View of Spacecraft Mockup within DFAT Speaker Circle ........................ 24

Figure 2-8. (a) Side view of test article showing Spacecraft Bus and adjacent + Y SA Panels (b)

Detail view of microphone and accelerometer used for data recovery ........................................ 24

Figure 2-9. (Top left) $+X$ Side View, (Top Right) + Y Side View, and (Bottom) $+Z$ Top View of

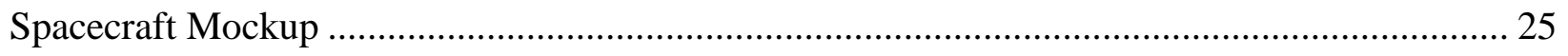

Figure 3-1. The Exterior Problem Given by the Helmholtz equation........................................... 32

Figure 3-2. (a) Spacecraft Mockup FEM and (b) Mesh extracted for the coupled FEM-BEM

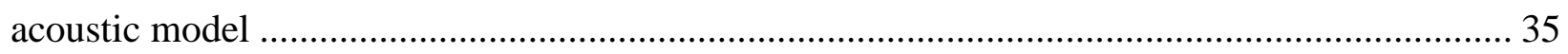

Figure 3-3. Cutaway view of Solar Array Panel Mesh in the Spacecraft Mockup FEM ............. 36

Figure 3-4. (a) FE Mesh used to transfer structural to the acoustic model and (b) overlaid BE Mesh

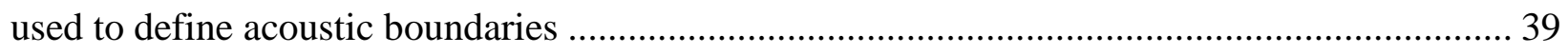

Figure 3-5. Enclosed BE Representation of Spacecraft Structure ……....................................... 40

Figure 3-6. Analytical Structural Response Measurement Locations ............................................ 45 
Figure 3-7. Analytical SPL Recovery Locations

Figure 4-1. Trade Study Map 49

Figure 4-2. Accelerometer on SA Panel 1 next to M-22 Microphone: Comparison of Test Data to FEM-BEM Prediction 50

Figure 4-3. M-22 Microphone between Bus and SA Panel 1: Comparison of Test Data to FEMBEM Prediction 51

Figure 4-4. ASD Predictions for Different Locations on Panels $1 \& 2$.................................. 52

Figure 4-5. SPL Predictions for Different Locations in Regions 1 \& 2 .................................. 52

Figure 4-6. Analytical Prediction of Modal Coupling between Structural and Acoustic Modes at Test Measurement Points..... 54

Figure 4-7. ASD Prediction at Test Accel Location for varied $d_{1}$ 55

Figure 4-8. ASD Prediction at Panel 1 Center for varied $d_{1}$ 56

Figure 4-9. ASD Prediction at Panel 1 Top Corner for varied $d_{1}$ 56

Figure 4-10. ASD Prediction at Panel 2 Center for varied $d_{1}$ 57

Figure 4-11. ASD Prediction at Panel 2 Top Corner for varied $d_{1}$ 57

Figure 4-12. SPL Prediction at Test Microphone Location for varied $d_{1}$ 59

Figure 4-13. SPL Prediction in the Center of Region 1 for varied $d_{1}$ 59

Figure 4-14. SPL Prediction at Corner of Region 1 for varied $d_{1}$ 60

Figure 4-15. SPL Prediction in the Center of Region 2 for varied $d_{1}$ 60

Figure 4-16. SPL Prediction at Corner of Region 2 for varied $d_{1}$. 61 
Figure 4-17. ASD Prediction at Test Accel Location for Varied $\mathrm{d}_{2}$......... 62

Figure 4-18. ASD Prediction at Panel 1 Center for Varied $\mathrm{d}_{2}$ 63

Figure 4-19. ASD Prediction at Panel 1 Top Corner for Varied $d_{2}$ 63

Figure 4-20. ASD Prediction at Panel 2 Center for Varied $d_{2}$ 64

Figure 4-21. ASD Prediction at Panel 2 Top Corner for Varied $d_{2}$ 64

Figure 4-22. SPL Prediction at M-22 Microphone Location for Varied $\mathrm{d}_{2}$. 65

Figure 4-23. SPL Prediction in the Center of Region 1 for varied $\mathrm{d}_{2}$ 66

Figure 4-24. SPL Prediction at Corner of Region 1 for varied $\mathrm{d}_{2}$ 66

Figure 4-25. SPL Prediction at the Center of Region 2 for varied $d_{2}$ 67

Figure 4-26. SPL Prediction at Corner of Region 2 for varied $d_{2}$. 67

Figure 4-27. Analytical Coupling between Panel 1 Structural Modes and Region 1 Cavity Modes for varied $\mathrm{d}_{1}$ 68

Figure 4-28. ASD Prediction at Solar_Array_D Accelerometer Location for Varied Damping Loss Factor Applied Across All Frequencies 70

Figure 4-29. ASD Prediction at Solar_Array_D Accelerometer Location for Different BEM Mesh Sizes 72

Figure 4-30. SPL Predictions at M-22 Microphone Location for Different BEM Mesh Sizes .... 72 Figure 4-31. SPL Prediction and BEM Solution CPU Time for Different BEM Mesh Sizes ...... 73

Figure 4-32. (a) 2" BE Mesh, (b) 6" BE Mesh, and (c) 10" BE Mesh 73 


\section{Introduction}

The study of acoustics, or the behavior of sound interacting with some medium, has several design applications ranging from musical instruments to conference rooms and helicopter rotor blades. Often, the desire is to characterize the acoustic field across a variety of frequencies and understand how mechanical energy is transmitted to structures. This is a very important consideration for spacecraft that experience intense acoustic noise at launch. The motivation for the research performed is given in this chapter. Spacecraft acoustic environments are subsequently defined and their fundamental characteristics are presented. Different acoustic analysis techniques can be employed, depending on the scope of the analysis and the frequency range of interest; these are presented and discussed. Much testing and analysis has been devoted in recent years to the accurate prediction of acoustic environments, with enhancements in computational power and modeling strategies resulting in improved analytical correlation with test data. In this work, the results of acoustic analysis are compared with the test data and the two primary forms of acoustic testing, including the method referenced in this work, are introduced.

\subsection{Motivation}

One of the overarching objectives in spacecraft structural design is to maximize strength and stiffness while minimizing mass; the associated design parameter is often quantified as the strength-to-weight and stiffness-to-weight ratio of a structure. This promotes structural integrity while reducing launch costs and improving spacecraft performance. However, the lightweight structures that result from this design objective, such as solar arrays, sandwich panels, and antenna reflectors, are often susceptible to the intense acoustic loads encountered in the early launch and ascension phases when the spacecraft is retained in the launch vehicle fairing. In many cases, sensitive components such as control system boxes, antennas, and cameras are attached directly to 
these lightweight structures and/or located in crowded internal compartments where the acoustic environments are magnified with respect to the ambient field. The acoustic pressure loads on the spacecraft primary structure produces random vibrations that can damage these components and potentially compromise their functionality and modal coupling between large lightweight structures and the attached hardware can result in vibration levels that exceed design specifications. For this reason, there is a continuous design initiative to tailor the volume of cavities and the corresponding layout of the interior components such that these random vibrations are kept within the design limits.

Often, the acoustic environments developed in spacecraft cavities are more intense than the ambient acoustic field in the fairing due the buildup of air pressure. It is of interest to understand what contributes to these pressure maxima at specific frequencies, observe at what frequencies these maxima occur, and identify design parameters that can be tailored to reduce these acoustic energy levels. While there is a general theoretical understanding for what influences the acoustic field characteristics in a confined volume and how modal coupling between structures can influence their dynamic responses to excitation, predicting how these behaviors will manifest themselves in complex structures is not always easy.

A variety of advanced computational tools using purely FEM, coupled FEM-BEM, or SEA modeling approaches have been developed to analytically predict acoustic environments in and around spacecraft assemblies with varying levels of fidelity. The analysis methodology can vary, but the consistent aim is to better inform the design of spacecraft before testing. In past decades, the acoustic environment within spacecraft compartments for new designs could not be assessed until the fully assembled structure underwent acoustic testing, at which point it would be hard to make significant design changes. In modern times, acoustic analysis is conducted as a final part of 
the design process before testing to simulate the acoustic environment and predict the corresponding structural responses. With the rapid increase in computational efficiency in recent years, several iterations of a spacecraft design can be assessed for a given acoustic excitation.

In standard acoustic testing procedures, the acoustic field levels are controlled and monitored by microphones situated around the periphery of the spacecraft, but sometimes additional microphone channels are used to monitor sound levels inside the spacecraft or between closely-spaced components in an effort to characterize local variations relative to the ambient field and how these could affect structural responses. This work is built around one such investigation where the region of interest is the tight gap (on the order of a few inches) between large sandwich panels attached to the exterior of the spacecraft bus. While the distances between these panels in the testing configuration are fixed, future spacecraft may have variations in spacing between panels, arrays, and other appendages and the changes in the local acoustic environment sensitive to these distances are of interest. In addition, there is a desire to understand how well conventional analysis techniques can predict these test measurements and observe what modeling strategies and assumptions contribute to prediction improvements. This work takes the form of a trade study where a baseline model is established that provides acceptable prediction of the test data and trades are conducted to investigate the sensitivity of the analytical solution to changes in spacing between spacecraft panels, damping assumptions, and model fidelity.

\subsection{Spacecraft Acoustic Environments}

The modern spacecraft contains multiple internal cavities that house important componentry and hardware sensitive to vibration. As spacecraft are launched and ascend through the atmosphere, they are subjected to a variety of dynamic load environments [1]; some typical examples are sine vibrations at low frequencies, vibroacoustic loads and random vibrations at mid-range frequencies, 
and transient shock loads at high frequencies. Vibroacoustic loads are imparted on spacecraft structures as a result of acoustic excitation and are manifested in the form of random vibrations. Acoustic excitations are produced by engine noise during ignition and lift off and turbulence from aerodynamic loads on the ascending launch vehicle; this transfer of mechanical energy creates fluctuating pressure loads on the spacecraft structure with intensity varying with frequency. The random vibrations generated from these sound pressures can damage sensitive spaceflight hardware and potentially compromise the mission of the spacecraft. Some potential problems resulting from exposure to these acoustic environments include component fatigue, connecting wire fracture, cracking of printed circuit boards, optical misalignment, and cracking of small structural elements [2]. For this reason, spacecraft acoustic environments are replicated through analysis and simulated in tests to verify that the corresponding dynamics of the structure pose no threat to mission success.

Extensive studies have been carried out to quantify the dynamic loads on the launch vehicle resulting from the acoustic pressure field generated by the rocket engine(s) during liftoff. The predictions for the acoustic pressures in a given region are usually specified in $1 / 3^{\text {rd }}$ octave bands. NASA cites four basic procedures for acquiring these predictions: (a) sound power techniques, scale acoustic models, (c) extrapolation techniques, and (d) direct measurements [3]. Expressions have been developed for early approximation of the sound power developed by one or more thrusters based on assumption for exhaust gas velocity and acoustic efficiency. The corresponding sound pressure level (SPL) at any location on the exterior of the launch vehicle can then be estimated as a function of distance and angle from the exhaust flow while also taking into account factors such as sound refraction from the launch pad and adjacent structures and spatial correlation of the sound field. Direct measurements of the acoustic loads on the exterior of the launch vehicle 
are sometimes made using strategically placed microphones mounted on the vehicle itself; these data points are used to confirm analytical predictions by measuring acoustic and aerodynamic loads during launch and ascent. Other direct measurements are made from ground-based instrumentation during launch and test firing of the rocket engines. For launch configurations where the launch vehicle and launch facility features are similar to those previously quantified, the acoustic environment for the new vehicle can be extrapolated.

Similar methods are employed for predicting the acoustic loads on the launch vehicle generated by fluctuating pressures imposed by the turbulent boundary layer (TBL) between the exterior surface of the launch vehicle and the ambient atmosphere as it ascends. These predictions are acquired using (a) boundary layer approximation techniques, (b) scale wind tunnel models, (c) extrapolation techniques, and (d) direct measurements [3]. The fluctuating pressure in the boundary layer is approximated as a function of the flight dynamic pressure, density of air at flight altitude, the launch vehicle free-stream air speed and Mach number, the geometry of the launch vehicle, and the boundary layer thickness. Scale wind tunnel models are made to characterize the aerodynamics of the launch vehicle and the overall fluctuating pressure level measured on the model can be scaled up for application to the full-size flight vehicle. In-flight measurement of the TBL fluctuating pressures can be achieved by mounting pressure transducers flush with the launch vehicle exterior or drilling holes that provide access to transducers mounted inside. The aerodynamic pressure loading measured on one launch vehicle can be scaled to another, assuming similar geometries in the area of interest.

The acoustic levels within a launch vehicle fairing are typically lower than those measured on the launch vehicle exterior as a result of noise reduction mechanisms such as acoustic blanketing and mechanical vibration transmission to other parts of the launch vehicle. Pressure transducers have 
been installed within a number of Space Shuttle payload bays and launch vehicle fairings to directly measure acoustic test levels. These measurements are also compared with the acoustic environment on the launch vehicle exterior to characterize the fairing with parameters including transmission loss and fill factor. The environment within the fairing of a given launch vehicle is typically specified in the launch vehicle user's guide. Figure 1-1 illustrates the relative sound pressure level around a rocket during the launch and ascension phases. 


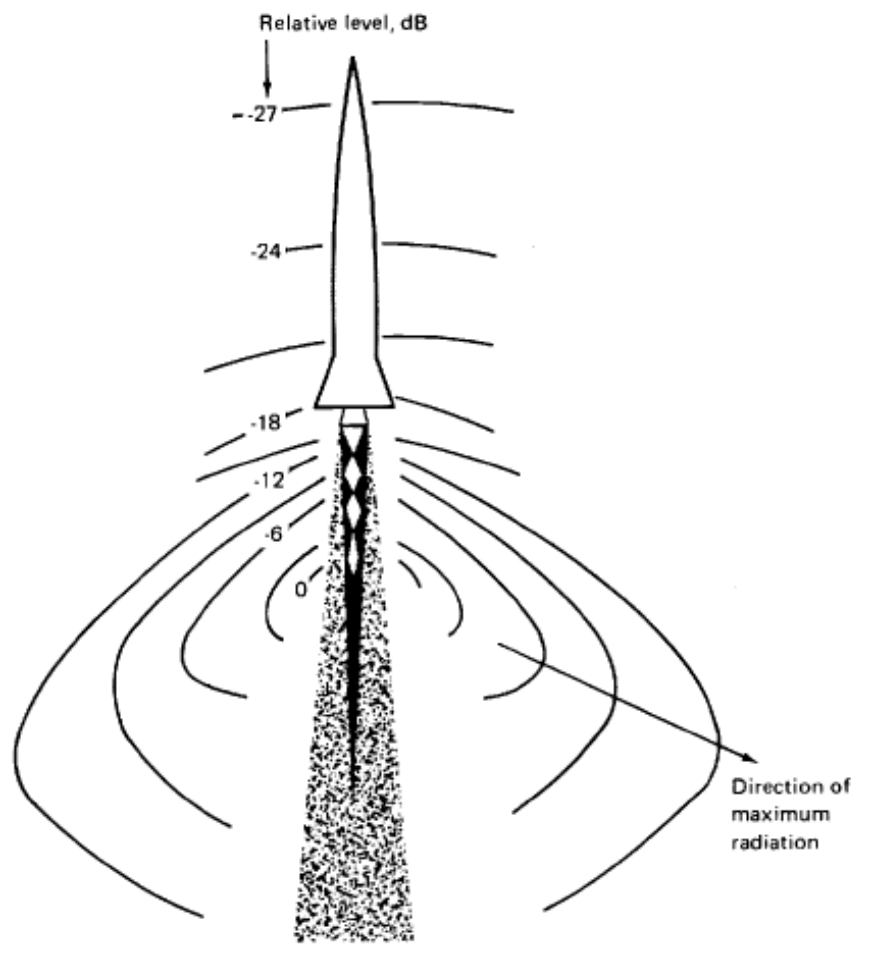

(a) In flight

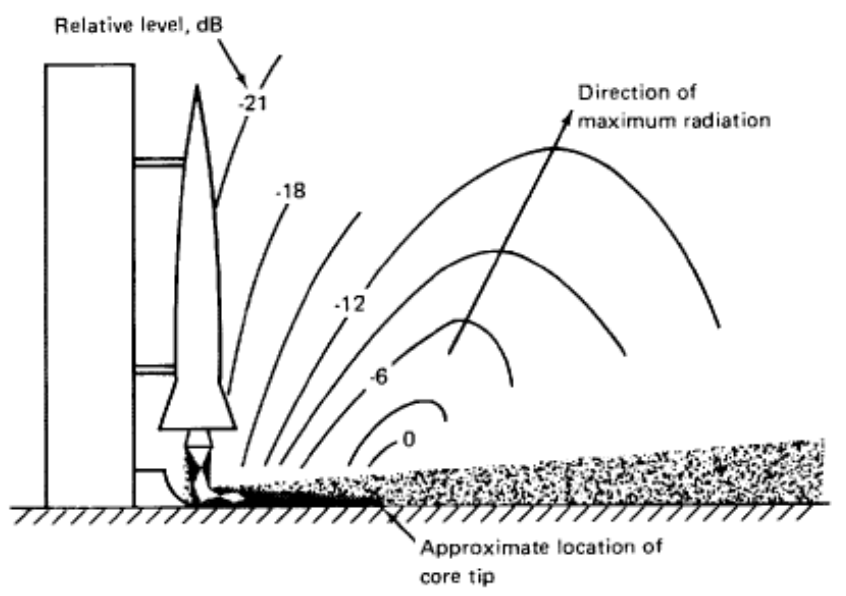

(b) At launch

Figure 1-1. Overall sound pressure levels around rocket for flight and launch cases [3]

\subsection{Acoustic Testing Methods}

Differing theories exist for the best way to represent the acoustic environment that a spacecraft sees in a launch vehicle fairing. As is the case with any other testing process for spaceflight hardware, the goal of the acoustic test is to qualify the structure to the launch vehicle acoustic environment in the safest way possible, at the lowest cost and at the lowest necessary levels, while 
meeting the test requirements and demonstrating capability with margin. A spacecraft is considered qualified to its launch vehicle acoustic environment if the random vibration responses of important components such as reflectors, antennas, solar arrays, and cameras are within their respective environment specifications for the appropriate test-simulated acoustic levels. Nowadays, a spacecraft can be qualified to its acoustic environment through Reverberant Field Acoustic Testing (RFAT) or Direct Field Acoustic Testing (DFAT). In both test methods, a series of speakers are used to project high intensity noise onto a test article placed in a test enclosure that maintains the desired acoustic environment for some duration ranging from 30 to 120 seconds. Both tests use microphones in the enclosure to measure the acoustic field around the test article and control power input to the speakers using a feedback loop. RFAT has been the standard testing methodology for decades, but DFAT has emerged in recent years as a viable alternative. RFAT is performed within a specially designed chamber with thick reflective walls for refracting the sound waves in the field at random orientations to create a uniform and diffuse acoustic field approximately similar to the launch vehicle fairing environment. In contrast, DFAT relies on a large enclosure of commercial-grade speakers to project sound directly onto the test article. The modularity of this testing method means that it can be performed in a variety of facilities as long as they have the necessary power and space requirements. DFAT also seeks to create a uniform acoustic field around the test article, but this field is not diffuse. In this work, data was acquired from a DFAT conducted on a spacecraft mockup model for comparison of test acoustic environment and structural response data with analytical predictions.

\subsection{Acoustic Analysis Techniques}

The analysis of acoustic environments can be divided into two primary domains: the low frequency coupled Finite Element-Boundary Element Method (FEM-BEM) domain and the high frequency 
Statistical Energy Analysis (SEA) domain. For the purposes of spacecraft acoustic analysis, low frequencies are usually considered to be at or below $500 \mathrm{~Hz}$, where the primary structural modes are located. In this range, there is sufficiently low modal content such that analysis by the deterministic FEM-BEM approach is appropriate. This approach is used to approximate the acoustic field and structural dynamics on frequency bands of low modal density (relatively low number of modes per frequency band) because it involves interpolation of modal responses between finite element and boundary element solutions at each combination of structural and acoustic modes in a given band. While the coupled FEM-BEM approach can theoretically be applied across all frequencies, it becomes inefficient at frequency bands of high modal density, where there are a plethora of structural modes in a given frequency range. At higher frequencies, interpolation between finite-element and boundary-element solutions for the system becomes computationally expensive and a deterministic analysis of the individual structural modes at these frequencies is usually unnecessary. Instead, assessment of the responses at these higher modes takes place on a statistical basis using SEA. A randomized process is assumed, and the structural system is divided into a collection of connected subsystems with a prescribed number of modes. Statistical averaging is used to capture the important modal characteristics of the system based on the energy stored among the connected subsystems and transferred between them.

In this work, the acoustic analysis frequency is limited to $400 \mathrm{~Hz}$ and the FEM-BEM solution is employed (which is standard practice). The modal density for the modeled structures in this frequency range is sufficiently low that a FEM-BEM solution is efficient; the theory and methodology employed for the FEM-BEM solution is discussed in subsequent sections. 


\section{Acoustic Testing}

In this chapter, the two prevailing methods for spacecraft acoustic testing, Reverberant Field Acoustic Testing (RFAT) and Direct Field Acoustic Testing (DFAT), are presented and their fundamental characteristics are compared. Then a detailed description of the Direct Field Acoustic Testing method is given that highlights the test configuration, important characteristics of the direct acoustic field, and parameters of interest. This is followed with an outline of the testing procedures that were used to acquire data points for validating analytical results presented in Chapter 4.

\subsection{State of the Art}

Nowadays, several tests are required by space agencies such as NASA and ESA to qualify spaceflight hardware for the intended mission. The goal of these tests is to effectively simulate the most intense service environments that the spacecraft and its subsystems will see over its operational life and demonstrate that the vehicle will maintain structural integrity and operate as planned under any of these circumstances with margin [1]; some examples are static load testing, sine vibration, random vibration, acoustic testing, and thermal vacuum testing. There are standards in place for all of these tests in terms of test methodology, pre-test and post-test analysis, test levels and duration, and personnel requirements. Within the domain of acoustic testing, two primary methodologies exist: Reverberant Field Acoustic Testing (RFAT) and Direct Field Acoustic Testing (DFAT). RFAT is the conventional method and has been employed for some decades as a means for simulating a diffuse acoustic field approximately equivalent to the launch vehicle fairing environment experienced by the spacecraft. In RFAT, the spacecraft is placed within a large chamber with thick reflective walls and sound sources specially tailored to create a diffuse acoustic field. A diffuse acoustic field is defined as having a uniform distribution of sound intensity 
throughout the enclosed volume such that sound wave propagation in all directions is equally probable. As illustrated in Figure 2-1, the test article (the spacecraft) is suspended in the middle of the chamber and well separated from the nearest chamber surface; for the purpose of field uniformity, the test chamber volume is required to be at least ten times greater than the volume of the test article. Random acoustic excitation is supplied by air modulators with electrodynamic actuators receiving commands from the controller. The desired acoustic field spectrum is supplied to the controller in $1 / 3^{\text {rd }}$ octave bands, typically from $100 \mathrm{~Hz}$ to $10 \mathrm{kHz}$. Acoustic levels inside the room are monitored by two or more Control microphones that participate in a feedback loop to adjust the input at the modulators. This test configuration is capable of producing overall sound pressure levels of 150 to $160 \mathrm{~dB}$ in any empty room. [1]

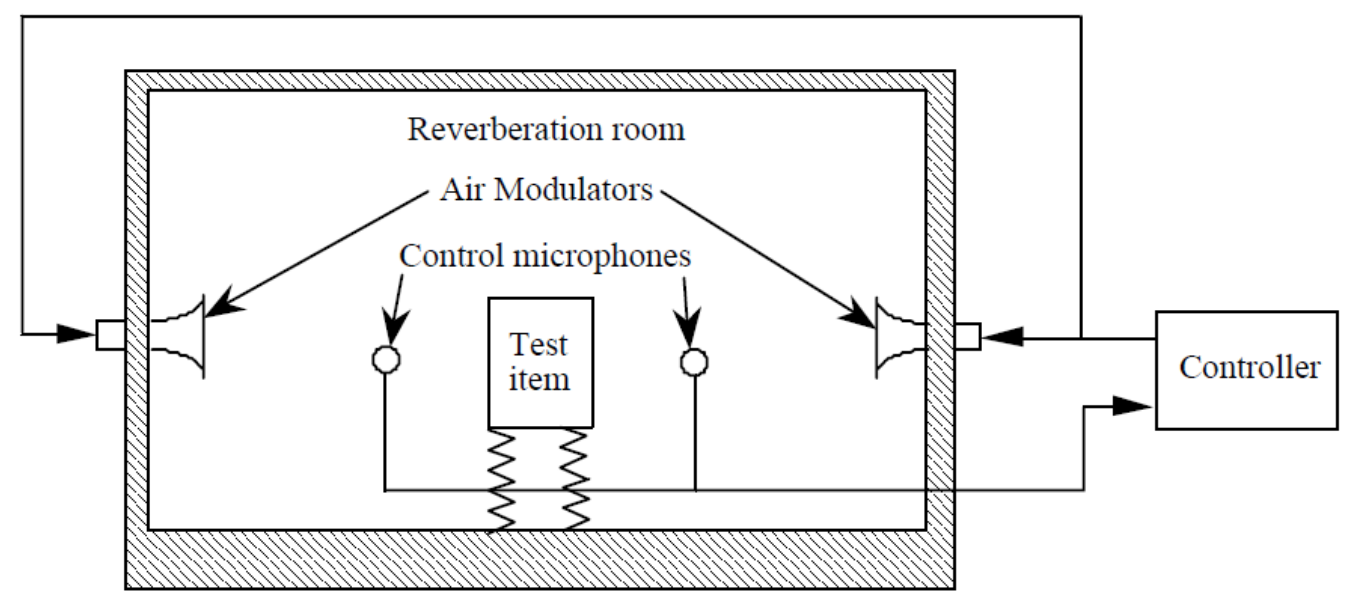

Figure 2-1. Diagram of Reverberant Chamber Test Configuration [1]

DFAT provides an alternative to RFAT in terms of both convenience and the method of acoustic impingement upon the test article. In contrast to RFAT, DFAT is not bound to a specially designed chamber; it consists of a modular arrangement of loudspeakers and amplifiers that can be set up in just about any facility with the space and power requirements where stacks of loudspeakers are positioned circumferentially around the test article to create an enclosure. Currently, private vendors with the necessary equipment are able to conduct DFAT on site with the spacecraft 
manufacturer instead of transporting the spacecraft to a separate RFAT facility; the testing process lasts a few days and minimal transportation of flight hardware is required. As the name suggests, DFAT produces direct impingement of the sound waves onto the spacecraft and does not achieve the diffuse field provided by RFAT, although the goal is to control the test environment to levels as close to a diffuse field as possible. In a similar fashion to RFAT, the desired sound pressure level spectrum is supplied to the controller in $1 / 3^{\text {rd }}$ octave bands, from $20 \mathrm{~Hz}$ to $10 \mathrm{kHz}$. The current DFAT testing configuration is capable of producing an overall sound pressure level of 145 decibels for a 60 second duration. Despite its differences, DFAT has been shown to be a reliable alternative to RFAT for qualifying flight hardware. Table 2-1 summarizes the comparison of the two acoustic testing methods.

Table 2-1. Comparison of Acoustic Testing Methods

\begin{tabular}{|c|c|c|}
\hline Test Method & $\begin{array}{l}\text { Reverberant Field Acoustic Test } \\
\text { (RFAT) }\end{array}$ & $\begin{array}{l}\text { Direct Field Acoustic Test } \\
\text { (DFAT) }\end{array}$ \\
\hline Control Bandwidth [Hz] & $100-10,000$ & $20-10,000$ \\
\hline Maximum OASPL [dB] & $150-160$ & $145-147$ \\
\hline Test Configuration & $\begin{array}{l}\text { - Large enclosed chamber with } \\
\text { thick reflective walls } \\
\text { - Test article suspended in the } \\
\text { middle } \\
\text { - Air modulators in walls } \\
\text { provide excitation } \\
\text { - Test article surrounded by } \\
\text { Control microphones to } \\
\text { measure acoustic field }\end{array}$ & $\begin{array}{l}\text { - In a facility with the space and } \\
\text { power requirements } \\
\text { - Stacks of loudspeakers } \\
\text { arranged circumferentially } \\
\text { around the test article } \\
\text { - Test article surrounded by } \\
\text { Control and Monitor } \\
\text { microphones to measure } \\
\text { acoustic field }\end{array}$ \\
\hline $\begin{array}{l}\text { Acoustic Field } \\
\text { Characteristics }\end{array}$ & $\begin{array}{l}\text { - Field is incoherent and diffuse } \\
\text { chambermity governed by } \\
\text { capabilities and controlled } \\
\text { frequency bands } \\
\text { - Reflective walls create } \\
\text { multiple plane waves having } \\
\text { fixed amplitudes and random } \\
\text { phase }\end{array}$ & $\begin{array}{l}\text { - Field uniformity governed by } \\
\text { number and positioning of } \\
\text { Control microphones } \\
\text { - Field is direct, with some } \\
\text { coherence depending on } \\
\text { speaker stack configuration } \\
\text { - Radially symmetric plane } \\
\text { waves around test article with } \\
\text { fixed amplitudes and } \\
\text { consistent phase }\end{array}$ \\
\hline
\end{tabular}




\subsection{Direct Field Acoustic Testing (DFAT)}

DFAT provides a series of advantages over the conventional RFAT alternative, including schedule flexibility, reduction of risks associated with transportation of flight hardware, and lower cost. One of the primary benefits of this newer method is its ability to accommodate larger spacecraft assemblies that might not be suited for a reverberant chamber. Figure 2-2 depicts the current DFAT speaker arrangement around the test article. The system consists of several commercial speakers accompanied by a set of high-powered stereo amplifiers, an array of microphones positioned around the test article, and a multi-input-multi-output (MIMO) control system.

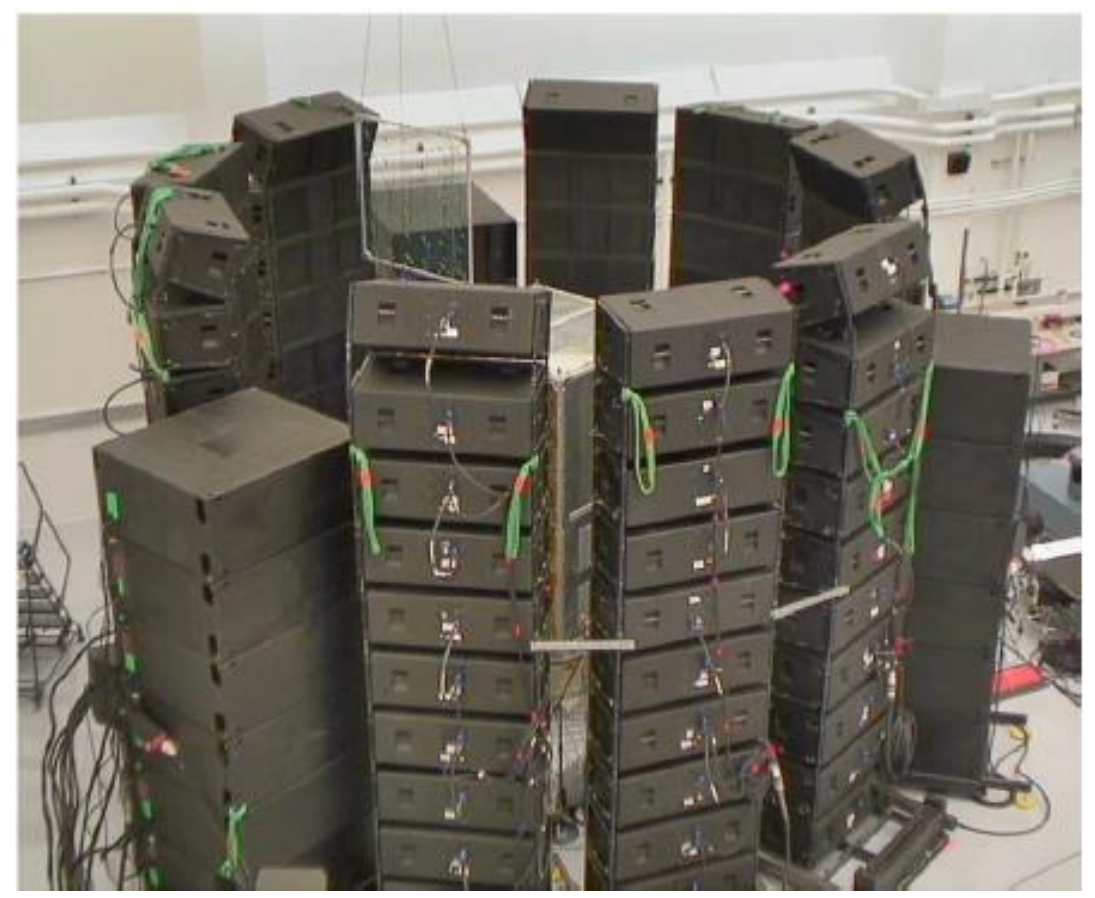

Figure 2-2. DFAT Speaker Arrangement around Spacecraft [5]

Several control microphones (at least 12) are placed circumferentially around the spacecraft as shown in Figure 2-3, either suspended from the ceiling or supported on stands. The number and spacing of the microphones is adjusted to accurately represent spatial variations in the sound field over all controlled frequency bands so that the average sound pressure level measured at all the control points approximates the desired test levels. The radial alignment of control microphones 
with respect to the loudspeakers is randomized to prevent biasing resulting from wave interference in the test volume.

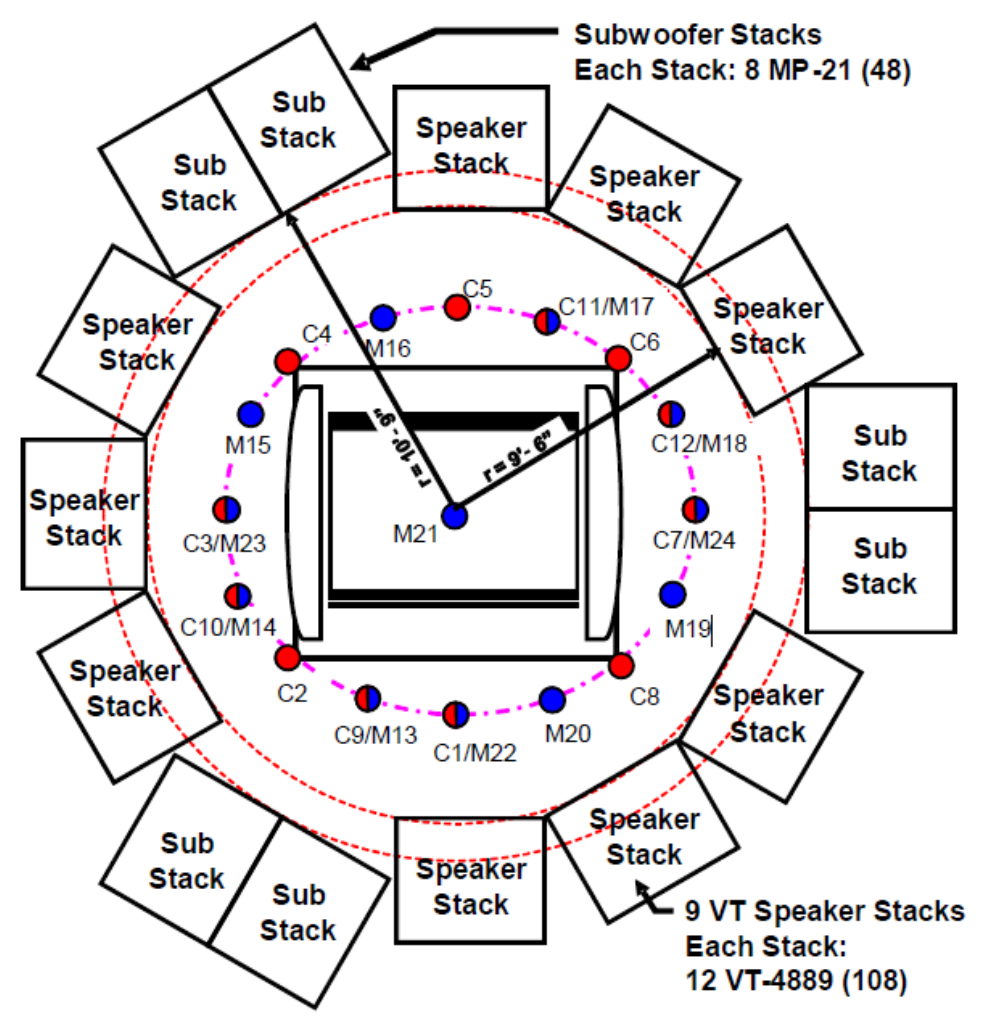

Figure 2-3. Floor Plan for DFAT with Loudspeaker and Microphone Arrangement [5]

The MIMO control system takes input from multiple independent drive signals at the microphones to govern output at the amplifiers and speakers. Using a fully populated spectral density matrix, the control algorithm allows the user to input magnitude, phase, and coherence specifications with tolerance bands on each. Previous control strategies used a single-input-single-out (SISO) controller to drive the acoustic field input based on the composite average sound pressure level seen by all control microphones, but this averaging method produced a highly coherent acoustic field that allowed for large variations at the control points and did not necessarily reflect a uniform field. The MIMO controller mitigates field coherence by allowing narrow-band control of the speaker output at discrete locations. [6] 
It is important to note the distinction between the sound field properties of uniformity and coherence. A uniform sound field is one in which the intensity of the sound field on a given frequency band is consistent in all directions. Coherence refers to the alignment of sound waves within the sound field; for sound waves of the same amplitude and frequency having constant phase, overlap can cause constructive or destructive interference. This interference can result in both over and under-testing of the spacecraft in the different frequency bands. The goal of the DFAT is to produce an incoherent, yet uniform acoustic field around the test article across all $n^{\text {th }}$ octave bands - a low coherence most accurately represents the launch vehicle fairing environment where the sound field consists of multiple superimposed sound waves at random orientation, frequency, and phase. Improvements in control methodology have led to increased field uniformity in DFAT, with the ultimate goal of creating an acoustic field as close to a diffuse acoustic field as possible.

An important consideration in the DFAT configuration is the structural-acoustic modal coupling that can occur resulting from standing waves. Standing waves are defined as periodic waves with fixed amplitudes in space caused by progressive waves of the same frequency and kind characterized by fixed nodes at locations where the amplitude is a minimum. They are created in a DFAT due to the radial symmetry of the speaker circle. These acoustic modes are important because they extend up to a few hundred $\mathrm{Hz}$, where modes of many spacecraft structural components are located [5]. Coupling between the acoustic and structural modes can cause significant increases in structural response and should be anticipated.

The phenomenon of acoustic/structural coupling at the standing wave modes in a reverberant chamber was explored by Kolaini and O'Connell [7] by studying the responses of lightweight reflectors at frequencies near the chamber modes-significant response variations were observed 
at these frequencies. NASA reached similar conclusions by tuning the dimensions of an aluminum panel to couple with the chamber modes. They found that an accelerometer on the panel positioned close to the monitor microphones showed significant response variations of up to $20 \mathrm{~dB}$ when the panel structural mode aligned with one of the standing waves [5].

Kolaini et al. [8] analytically modeled the interior of the DFAT test configuration as an open cylindrical cavity with rigid walls. They studied the spectral density of the sound pressure across the frequency spectrum to compare theoretical predictions of the cavity modes with test data points from a test setup of similar size. Analytical predictions for two cavity mode shapes are shown in Figure 2-4 - the corresponding frequencies are consistent with a number of structural modes observed in testing. The important distinction was made between interference patterns resulting from the control scheme and standing waves, which are independent from the controller input.
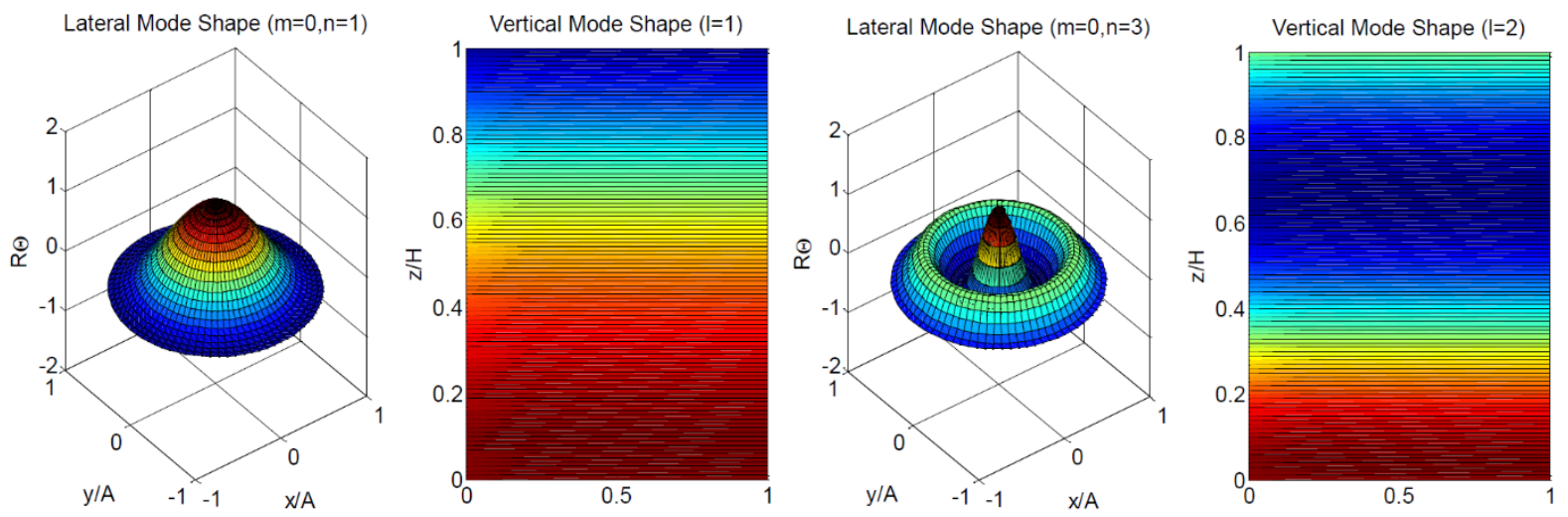

Figure 2-4. Predicted cylindrical cavity modes at (a) $88 \mathrm{~Hz}$ and (b) $237 \mathrm{~Hz}$ [7]

Rouse, Mesh, and Stasiunas [9] explored the spatial variation in the direct acoustic field during a DFAT in both pure-tone and broadband fields for different numbers of plane waves with constant phase and compared this with corresponding results for a RFAT diffuse field with plane waves of random phase. In agreement with the results of Kolaini et al., they found that the direct acoustic field exhibited significant spatial variation characterized by an amplitude peak at the center of field 
surrounded by concentric rings of local maxima and minima. Rouse et al. also studied the response of a thin simply-supported beam modeled at different orientations in the center of the acoustic field and showed that the structural response was sensitive to its orientation in the direct acoustic field due to the deterministic arrangement of plane waves. Van Dyke [10] studied the SPL variation in the DFAT environment by placing a $6 \times 6$ array of microphones at several locations in the acoustic field. These discrete measurements were used to create a contour map of the spatial gradient for the acoustic field at control microphone locations. Results showed evidence for the presence of standing waves, but it was difficult to achieve direct correlation with the findings of Kolaini et al. It was suggested that field control difficulties at certain frequencies might be due to the control microphones being located in a depression in the acoustic field.

With these considerations in place, there is a strong need for reliable analytical prediction of the acoustic environment for any spacecraft test. Heritage test data is used to analytically re-create the empty-chamber acoustic field and map the acoustic modes and this can be combined with an acoustic analysis of the spacecraft to optimize the test setup (locations of the speakers) and acoustic field levels in addition to orientation of the spacecraft to ensure that responses of sensitive hardware meet specifications. In this work, the acoustic field of the entire test volume is not modeled, since the area of interest is confined to the region between panels.

\subsection{Test Procedure}

The following steps are consistent with the recommendations set forth in NASA-HDBK-7010 [5] and MIL-STD-810G [2]. A general description is provided of the test procedure that was conducted to acquire data points presented later in this work. 


\subsubsection{Pre-test Preparation}

The first step in any spacecraft test is pre-test preparation. For an acoustic test, this means analytical characterization of the structural responses on spacecraft secondary structures in response to random excitation. It is recommended to generate analytical models of the acoustic test volume both with and without the test article to first identify acoustic chamber modes corresponding to standing waves and interference patterns and then assess what structural modes of the test article may be likely to couple with these chamber modes. Depending on the frequency range of interest, BEM and/or SEA models can be used for this analysis. Spacecraft structures most susceptible to acoustic loads, such as reflectors, large sandwich panels, antennas, and solar arrays are accounted for in this model and their dynamic responses are recovered. The acoustic input spectrum for these analytical models is derived from the launch vehicle user's guide specified

acoustic environment, since similar levels will be used in testing. Figure 2-5 gives an example of the acoustic environment specification for some typical launch vehicles. 


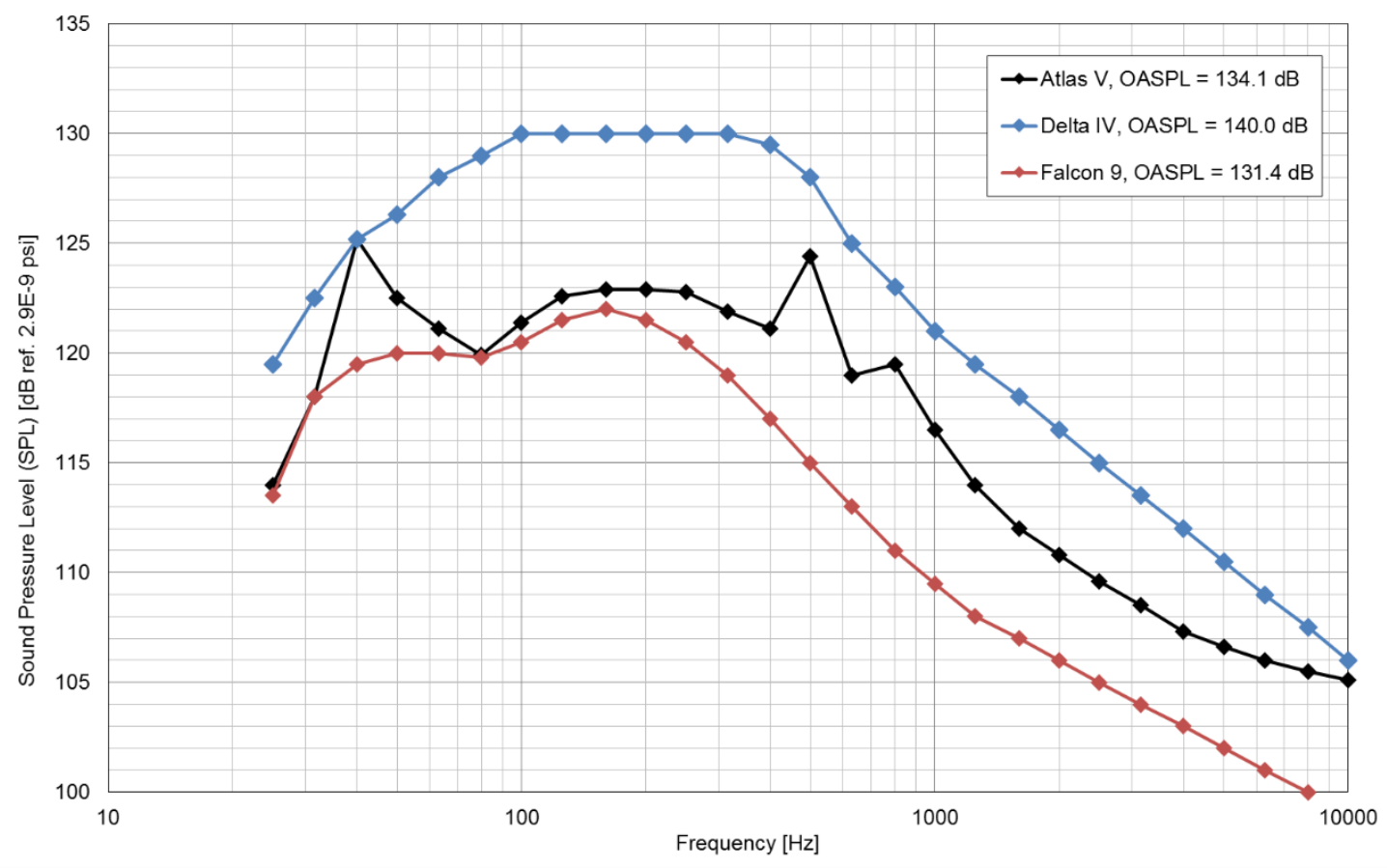

Figure 2-5. Typical Launch Vehicle Fairing Acoustic Environments [11]-[13]

Analytical random vibration responses of these structures are compared against their random vibration environment specifications to predict any out-of-tolerance exceedances; structural components showing high analytical response predictions are instrumented with accelerometers to be monitored during the acoustic test. Information from these pre-test analyses can be used to inform the placement of control microphones for adjusting the acoustic field levels during testing and the orientation of the spacecraft within the speaker circle. The methodology used for creating and evaluating these analytical models is detailed in Chapter 3.

\subsubsection{DFAT Test Setup Preparation}

The DFAT configuration is mapped out in a manner similar to the schematic in Figure 2-3 and the speakers, amplifiers, and associated hardware are assembled in the test area based on this layout. The test article is surrounded on all sides by speaker stacks to a height of at least $1 \mathrm{~m}(3.28 \mathrm{ft})$ above the top of the article. The test article is mounted on a wheeled dolly and secured at its base 
to a metal fitting using a clamp band similar to the mounting condition used in the launch vehicle fairing. Control and monitor microphones are placed inside the test enclosure, either supported on stands or suspended from above in locations specified by the test procedure. The radial distance from the control microphones to the test article should be between $0.5 \mathrm{~m}(1.64 \mathrm{ft})$ and $0.75 \mathrm{~m}(2.5$ $\mathrm{ft}$ ) at a height centered along the mid-height of the test article, varying up and down by about oneeighth of the test article height. Control microphone locations are specified in an effort to promote field uniformity while mitigating coherence. Because of the high noise levels, the test is monitored and controlled by personnel in an adjacent room providing some amount of noise reduction. Initial system checkouts are performed to confirm that all test equipment, including speakers, amplifiers, and microphones performs nominally. [2]

\subsubsection{Acoustic Field Shaping}

After verifying that all of the speakers, amplifiers, and microphones are operating correctly, field shaping runs are performed using a mass mockup resembling a heritage spacecraft. The shaping runs are performed to the same levels as those planned for the flight hardware. Three 60-second test runs are performed at levels scaled relative to the spacecraft flight-level acoustic environment in decibels $(\mathrm{dB}):-12 \mathrm{~dB},-6 \mathrm{~dB}$, and $0 \mathrm{~dB}$ in ascending order. The control and monitor microphones are initially positioned around the mass mockup as defined in the test procedure, but these can be moved between test runs and any changes in the microphone arrangement are noted in the as-run test procedure. The goal of these shaping runs is to measure the acoustic field created around the test article corresponding to the specified spacecraft acoustic environment. While the mass mockup is instrumented with accelerometers to measure structural vibrations, the emphasis for these simulation runs is on the variances in SPL readings between control microphones at each $1 / 3^{\text {rd }}$ octave band; the goal is to keep all of the control microphone levels within some tolerance of the 
specified environment, usually $\pm 3 \mathrm{~dB}$. The acoustic field in the enclosed test configuration is significantly influenced by the presence of the test article and the field levels at the control microphone locations around the spacecraft are monitored to verify that the desired acoustic field can be created for a test article of comparable size to the spacecraft. Preliminary testing allows evaluation of any spatial fluctuations in sound pressure levels that could result in over/under testing of the structure. These shaping runs are considered successful if all of the test equipment functions correctly and the acoustic field measured at the control microphones is within the specified tolerances at the $0 \mathrm{~dB}$ level for all frequency bands, indicating that the desired acoustic test environment can be created for the subsequent spacecraft tests. It should be noted that the test data used for this research was acquired from one of these $0 \mathrm{~dB}$ level simulation runs.

\subsubsection{Spacecraft Testing}

The acoustic tests for the spacecraft are conducted in a similar manner to the shaping runs, with test runs at multiple levels relative to the spacecraft acoustic environment. The number of intermediate tests conducted and their corresponding duration can vary (usually between 30 and 60 seconds) based on the test requirements such as the target OASPL, but the objectives are consistent: to ensure that the acoustic field created around the spacecraft matches the desired levels within established tolerances and that the structural responses of sensitive components will conform to their specified random vibration environments. For a MIMO controlled test, typical test tolerances are $\pm 1 \mathrm{~dB}$ on the composite control average and $\pm 3 \mathrm{~dB}$ at each control microphone relative to the reference overall acoustic test level spectrum.

For some test level relative to the flight level acoustic environment specified in decibels, the corresponding SPL input profile over all controlled frequency bands is scaled by that amount; the MIMO controller adjusts the power input for the speakers to achieve these scaled levels. 


\subsubsection{Data Review and Post-Test Analysis}

A number of intermediate steps take place in the time between acoustic test runs. After an acoustic test, the speakers need time to cool down and the control system must be adjusted for the next test in the schedule. Data recovered from the recently completed test is reviewed. First, the SPL measured at each of the Control and Monitor microphones in the test over the controlled frequency bands is reviewed to confirm that the acoustic field was within tolerances over the entire spectrum. The SPL measurements at each of the microphone locations can be compared to discern any local anomalies in the acoustic field or pressure peaks/drops that could affect the structural random vibration environments.

Next, the random vibration responses recovered from all accelerometer channels are reviewed and compared with the relevant component random vibration environment specifications. Data recovered for all levels is scaled to normalize each response profile to the intended flight level for direct comparison. The random vibration response at each of these accelerometers is specified in terms of Acceleration (or Power) Spectral Density (ASD) and measured in units of $\mathrm{g}^{2} / \mathrm{Hz}$ - this measurement provides a normalized value for the acceleration as a function of frequency. The overall root mean square (RMS) acceleration measured in gs over the entire frequency spectrum is also recorded, which is useful for comparing the total energy present in response profiles. As multiple runs are conducted, these scaled random vibration responses are overlaid and compared to confirm consistent structural behavior. Significant alterations in the scaled structural response profile between runs can indicate some problem with the test configuration, such as a malfunctioning accelerometer, a detached accelerometer, or damage to some structural component of the spacecraft. Heritage datasets can also be employed for comparison if spacecraft with similar 
configuration to the current test article have been tested previously. Any anomalies in the test data are noted in the as-run test procedure.

After all test data has been reviewed, necessary adjustments are made to the test configuration, such modifying microphone positioning or adding accelerometers to the spacecraft. If all measured response data conforms to the relevant specifications, the test proceeds to the next level in the schedule. At the conclusion of the $0 \mathrm{~dB}$ (full-level) test run, the acoustic test is deemed successful if the desired acoustic field levels were achieved at all control microphones within tolerances and the random vibration responses of all monitored structural components met their respective environment specifications.

\subsection{Data Recovery}

The test data used in this work was acquired during the full level $(0 \mathrm{~dB})$ acoustic test on the spacecraft mass mockup illustrated in Figure 2-6. A monitor microphone was attached to a stand and inserted into the narrow space between the $+\mathrm{Y}$ bus panel and adjacent solar array panel to measure the sound pressure level in the cavity, as shown in Figure 2-7. An accelerometer was placed on the inner of the two solar array panels next to the microphone to measure the random vibration response of the panel during the test. Both instruments were wired to separate data acquisition systems and their respective response levels were measured throughout the 60 second test run. 


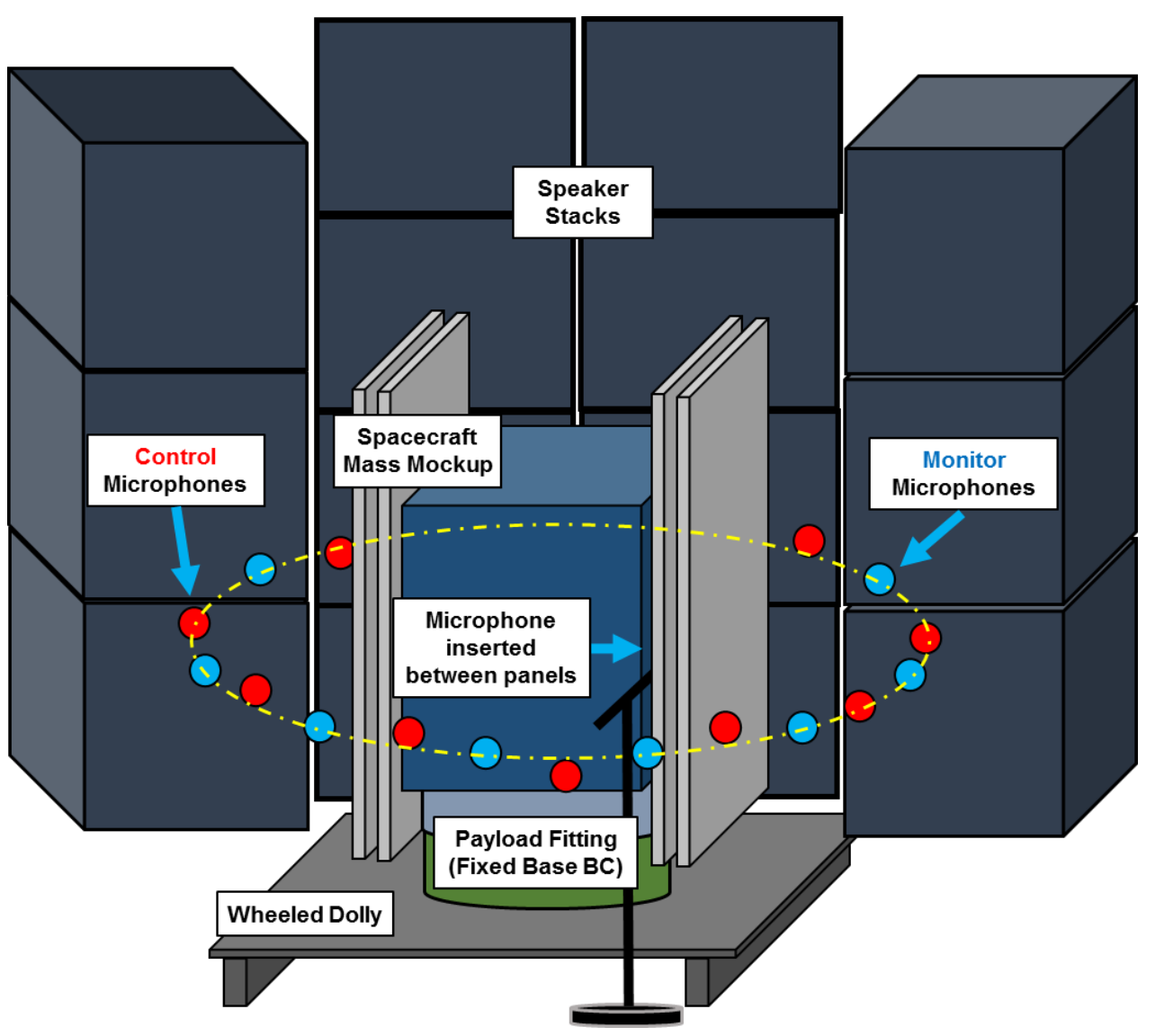

Figure 2-6. Cutaway View of Spacecraft Mockup within DFAT Speaker Circle
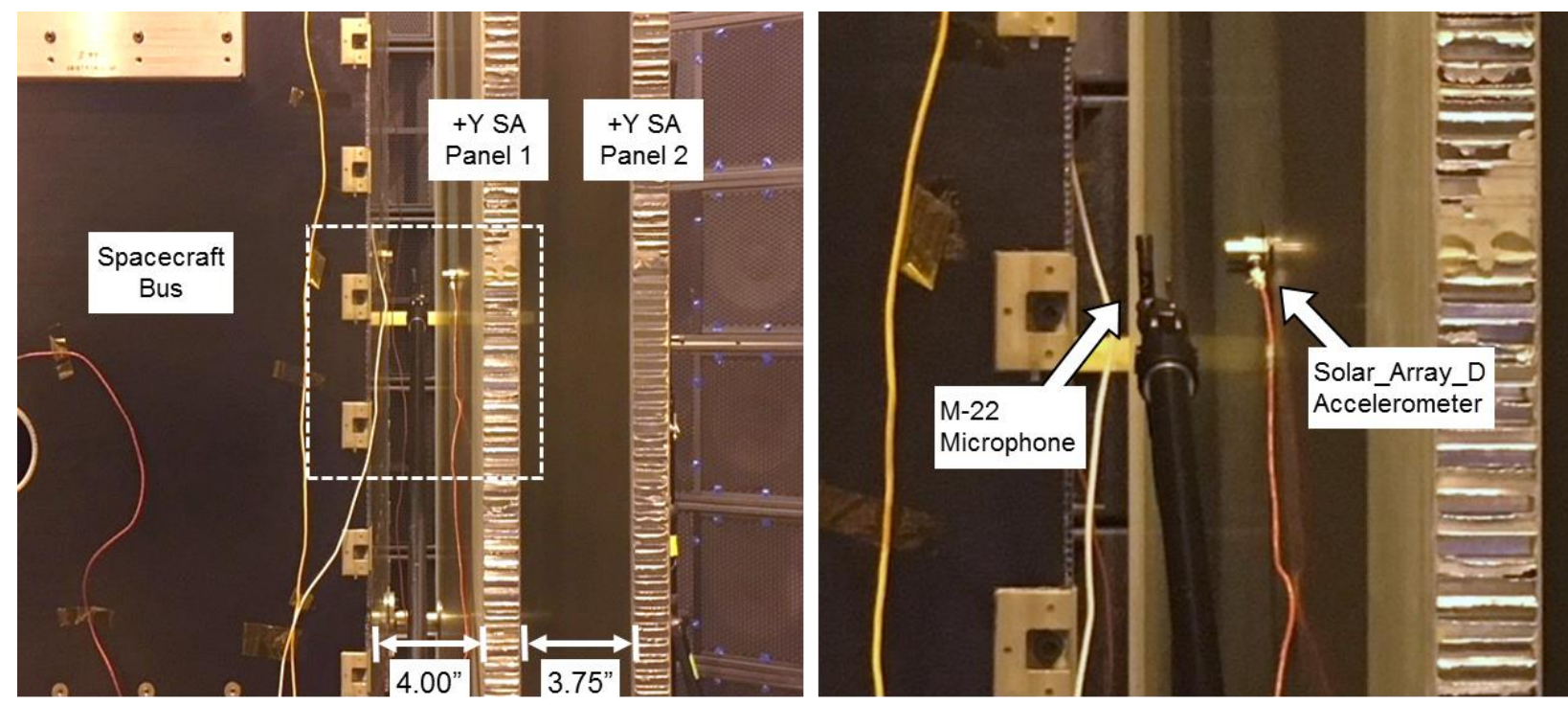

Figure 2-7. (a) Side view of test article showing Spacecraft Bus and adjacent +Y SA Panels (b) Detail view of microphone and accelerometer used for data recovery 


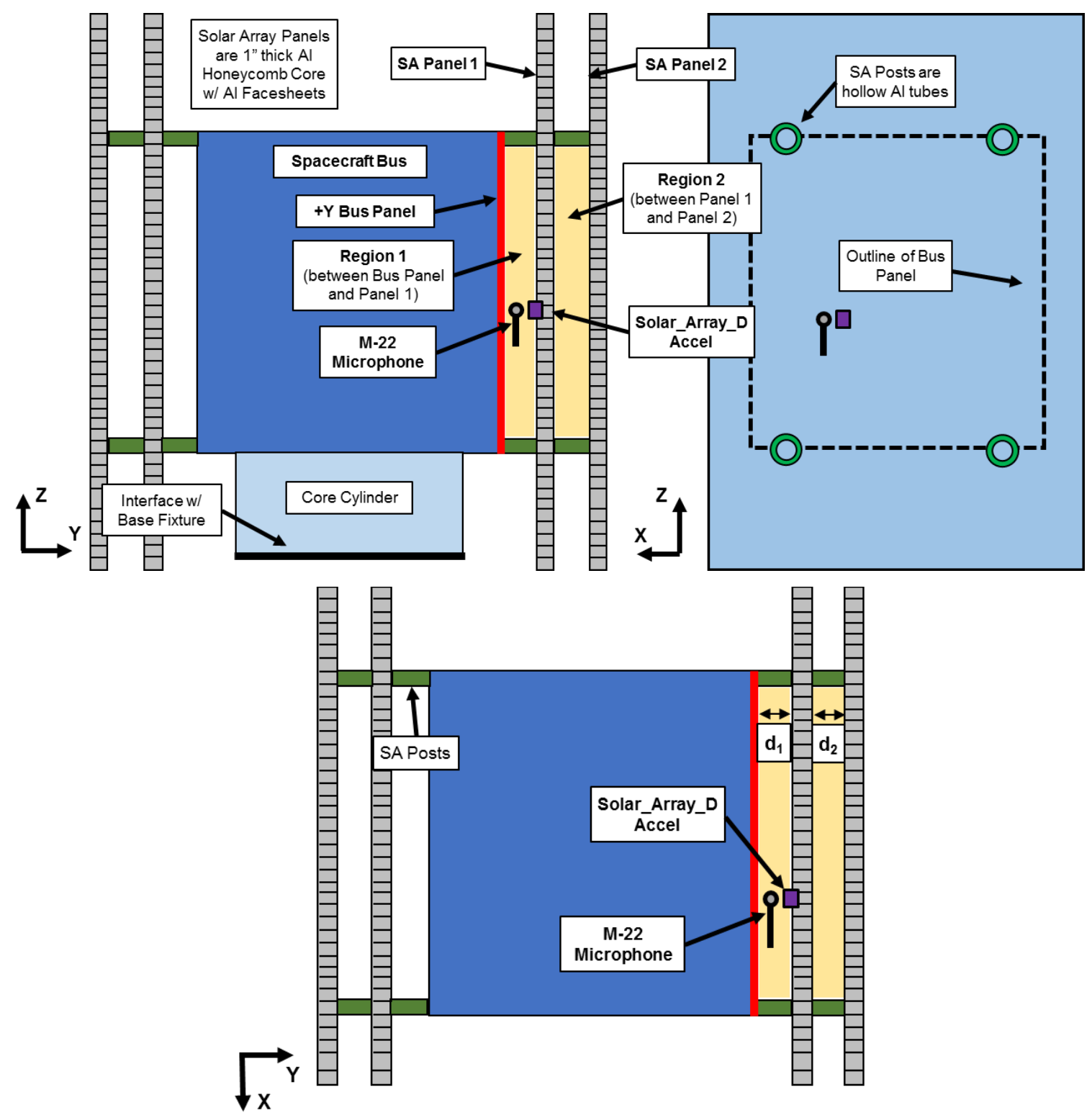

Figure 2-8. (Top left) + X Side View, (Top Right) + Y Side View, and (Bottom) + Z Top View of Spacecraft Mockup 


\section{Acoustic Analysis Using a Coupled FEM - BEM Approach}

In this chapter, the method used for the acoustic analyses performed is explained in depth. First, a literature review is presented to survey the development of the FEM-BEM analysis method and understand the motivation behind its employment; a review of some of the underlying theory is also provided. Next, the modeling and analysis process used to do this work is discussed in detail.

\subsection{Literature Review}

Today's acoustic analysis that uses powerful computational resources to simulate acoustic and random vibration environments for a wide range of spacecraft components has its roots in the 1960s, when electronic computers began to be employed to solve large matrix problems. BEM provides a series of advantages over a FEM approach to solve the acoustic fluid domain because it reduces a three-dimensional problem to one in two dimensions by only requiring discretization of the bounding surfaces instead of the entire volume. This become advantageous for problems involving infinite and semi-infinite fluid domains because after the discretized boundary is solved, the subsequent solution for some arbitrary point in the domain can be acquired. In addition, reducing the problem by one dimension usually results in decreased computational cost. These simplification motivated the development of BEM as alternative solution approach for boundaryvalue problems.

Cheng and Cheng [14] provide a survey of the mathematical foundation for the boundary element method and trace its key developments through the advent of the computer in the 1960s and 70s. Friedman and Shaw [15] solved the two-dimensional wave equation for the pressure and velocity fields resulting from a plane wave interacting with a cylindrical obstacle. The surface boundary was discretized in space and time and a double summation was derived to solve for the surface 
pressure loading for arbitrary geometries; this approach was applied for the case of a square box with rigid walls and showed good agreement with known analytical results. In similar fashion, Banaugh and Goldsmith [16] discretized the two-dimensional wave equation on several surfaces of arbitrary shape to solve for the acoustic field potential both on the surface and at the far field. It is noted that for a desired computational accuracy, the number of boundary segments must be increased in proportion to the incident wavenumber. In a similar manner, the number of boundary elements (analogous to mesh size) chosen for the BEM solution in this work is directly proportional to the maximum analysis frequency.

Chen and Schweikert [17] developed an analysis method for the boundary value problem of acoustic radiation from a shell in an infinite fluid medium. The numerical method developed used triangular shell elements to discretize the surface. It is noted that triangular elements are wellsuited to approximating surfaces of arbitrary geometry. Copley [18] developed a numerical technique to solve the acoustic problem on boundaries of vibrating surfaces using an interior version of the Helmholtz integral equation relating the surface pressure and normal velocity for field points strictly within the enclosed volume bounded by the surface. He also raised the issue of non-uniqueness in the solution of the integral equation, stating that a solution for the discretized pressure loading on the surface that satisfies the wave equation and Sommerfeld radiation condition given a specified normal velocity may not be unique. Schenck [19] proposed a Combined Helmholtz Integral Equation Formulation (CHIEF) that overcame numerical difficulties inherent in conventional integral equation solutions and avoided the problem of non-uniqueness identified by Copley. This formulation solves for the unique surface pressure distribution that satisfies both internal and external Helmholtz integral equations to predict the pressure anywhere in the exterior fluid domain. The procedure is implemented for computation as a Gaussian numerical quadrature 
over a series of discrete boundary elements and numerical examples are presented for rectangular and cylindrical structures with mixed boundary conditions.

Some 30 years later in 1997, Benthien and Schenck [20] surveyed existing approaches for solving the acoustic radiation and scattering problem, including the classic Helmholtz integral relations, exterior collocation and wave superposition methods, and modified versions of CHIEF and presented the relative pros and cons of each approach in terms of accuracy and efficiency for solving some simple cases. It was concluded that each of the surveyed methods has distinct strengths and weaknesses, but that the core CHIEF approach is still practical and efficient for many boundary value problems. It is noted that all of the integral equation techniques are most effective at low to moderate frequencies because as characteristic frequency increases, the mesh quality required becomes computationally expensive; the results of the mesh convergence study conducted in this work are consistent with this remark.

Pretlove [21], [22] investigated the vibration response of thin panels covering closed cavities as a reduced model with application to noise transmission and acoustic fields in enclosed regions. He modeled a closed box with a single flexible wall and the others assumed to be rigid. By varying the volume of the box with respect to the dimensions of the flexible panel, he observed two distinct cases: in the first, the panel modes were negligibly influenced by the cavity volume when the cavity depth was sufficiently large compared to the panel dimensions. In the second case, for a shallow cavity, the cavity modes acted as an acoustic stiffness on the order of the fundamental panel frequency and significantly influenced subsequent panel modes. It was concluded that for this second case of thin panels over shallow cavities, several in vacuo panel modes would be necessary to accurately characterize panel responses. Kopuz, Unlusoy, and Caliskan [23] applied a coupled FEM-BEM analysis approach to obtain structural modes and sound pressure levels for 
a similar simple open-ended box structure subject to sinusoidal forcing functions. Experimental results showed good agreement with analytical predictions.

A number of ongoing initiatives are present in industry to correlate analytical predictions for the RFAT and DFAT environments with test data. Tsoi, Kolaini, and Childs [24] used a coupled FEMBEM analysis procedure to predict the random vibration responses at multiple points on a composite reflector during a RFAT. The analysis was conducted for frequencies up to $300 \mathrm{~Hz}$ and showed good agreement with test data; this work demonstrated the effectiveness of analytical predictions for individual component testing prior to integration to the spacecraft. Ruess et al. [25] performed a coupled BEM-FEM analysis for a SGEO satellite subjected to the RFAT acoustic environment using an uncorrelated spacecraft FEM and demonstrated good correlation for structural responses at a point on the spacecraft radiator for frequencies up to $720 \mathrm{~Hz}$. The favorable analytical correlation was used to qualify the spacecraft for the prescribed acoustic noise environment. Gardner et al. [26] created a BEM model of the acoustic field in DFAT to investigate the field diffusivity based on the spatial distribution of speakers providing acoustic excitation. These results were compared against the simplified case of an ideal diffuse field modeled with an increasing number of plane waves. They showed that analytically reducing correlation (providing incoherence) between the speaker stacks provided better variation in the field. It was concluded that analytically mapping the acoustic field produced by different speaker arrangements can allow for qualification of acoustic test environments.

In recent years, Inoyama, Agarwal, and Stoumbos [27]-[29] have investigated the internal vibroacoustic environment in spacecraft during DFAT by comparing test measurements with analytical predictions using BEM and SEA. In the initial study [27], reduced BEM and SEA acoustic models of the spacecraft internal cavities were created and a FEM of the spacecraft under testing was used 
to supply normal modes for the structure. The BEM and SEA analyses were used to predict the internal cavity vibro-acoustic environment from 20 to $2,000 \mathrm{~Hz}$, in terms of SPL at points corresponding to microphone locations in the test. It was shown that the BEM was effective at predicting narrowband sound pressure spikes below $200 \mathrm{~Hz}$ with reasonable accuracy without correlated FE structural modes. In subsequent work [28], Inoyama et al. focused on improving BEM correlation with test data in the low-frequency regime below $400 \mathrm{~Hz}$. Analytical predictions of the internal cavity vibro-acoustic environment were improved by increasing the model fidelity; a detailed spacecraft FEM was coupled with the BE mesh and normal FE modes for the structure were obtained for frequencies up to $500 \mathrm{~Hz}$. In addition to measuring the SPL at different points in the internal cavities, structural responses were analytically measured on the cavity walls corresponding to accelerometer locations in the acoustic test. The model was separated into multiple acoustic domains to account for the internal cavities and external field environment. Commercially available NASTRAN software was used for the FE normal modes analysis and VA One software was used to model and solve the coupled acoustic problem; similar methodology is employed for the acoustic analysis performed in this work and described in subsequent sections.

\subsection{Theory}

The coupled acoustic boundary element-finite element problem has been studied extensively and the modeling of the fluid domain and fluid-structure interaction presented in this work is well documented in the literature. The intermediate boundary element solution is laid out in a manner consistent with the process described by Kopuz et al. [23], Djojodihardjo [30], and Holström [31], beginning with the formulation of the acoustic wave propagation in the field governed by the Helmholtz equation - this allows for calculation of the acoustic pressure loading on the fluidstructure boundaries. The intermediate solution for the structural dynamic problem is conducted 
in parallel using the finite element method. These intermediate solutions for the fluid boundary and structural dynamics are then brought together to solve for the system dynamics using a BEMFEM coupling method. For the exterior acoustic problem, consider a closed surface $S$, containing some interior volume $V_{\text {int }}$. The problem domain is the exterior volume $V_{\text {ext }}$ surrounded by the boundary $\Gamma$ as depicted in Figure 3-1. This method assumes that the acoustic field is composed of a series of time-harmonic plane waves consistent with the Helmholtz equation:

$$
\nabla^{2} p+k^{2} p=0
$$

where $p$ is the position and time dependent pressure field, $p=p(x, y, z, t)$ and $k$ is the wave number expressed as the angular frequency $\omega$ divided by the speed of sound waves in the fluid $c$ (air in this case). The corresponding boundary integral equation is given by the Helmholtz equation:

$$
C p(\boldsymbol{R})=\int_{S}\left(p\left(\boldsymbol{R}_{0}\right) \frac{\partial g}{\partial \hat{n}_{0}}-g\left(\left|\boldsymbol{R}-\boldsymbol{R}_{0}\right|\right) \frac{\partial p}{\partial \hat{n}_{0}}\right) d S
$$

where $\hat{n}_{0}$ is the unit normal vector on the surface boundary and $C$ varies with $\boldsymbol{R}$ such that

$$
C= \begin{cases}1, & R \text { in fluid domain } \mathrm{V}_{\mathrm{ext}} \\ \frac{1}{2}, & R \text { on smooth fluid-structure boundary } \mathrm{S} \\ 0, & R \text { outside fluid domain } \mathrm{V}_{\mathrm{ext}}\end{cases}
$$

with $\boldsymbol{R}$ representing some location in the fluid domain, $\boldsymbol{R}_{0}$ is a point on the closed surface boundary $S$, and $g$ is the free-space Green's function given by

$$
g\left(\left|\boldsymbol{R}-\boldsymbol{R}_{0}\right|\right)=\frac{e^{-i k\left|\boldsymbol{R}-\boldsymbol{R}_{0}\right|}}{4 \pi\left|\boldsymbol{R}-\boldsymbol{R}_{0}\right|}
$$

Because the Helmholtz integral equation satisfies the Sommerfeld radiation condition 


$$
\lim _{\left|\boldsymbol{R}-\boldsymbol{R}_{0}\right| \rightarrow \infty}\left|\boldsymbol{R}-\boldsymbol{R}_{0}\right|\left(\frac{\partial g}{\partial\left|\boldsymbol{R}-\boldsymbol{R}_{0}\right|}+i k g\right)=0
$$

on the exterior fluid boundary $\Gamma$ the BEM is capable of solving problems with unbounded (infinite) acoustic domains, which presents a significant advantage over a strictly FEM-based solution. Boundary conditions are assumed to be known on all surfaces in the fluid domain; either the pressure $p$ or the normal velocity $v$ is known at every point on the boundary, or some relationship between the two in the form of specific normal impedance given by $p / v$ [Holstrom].

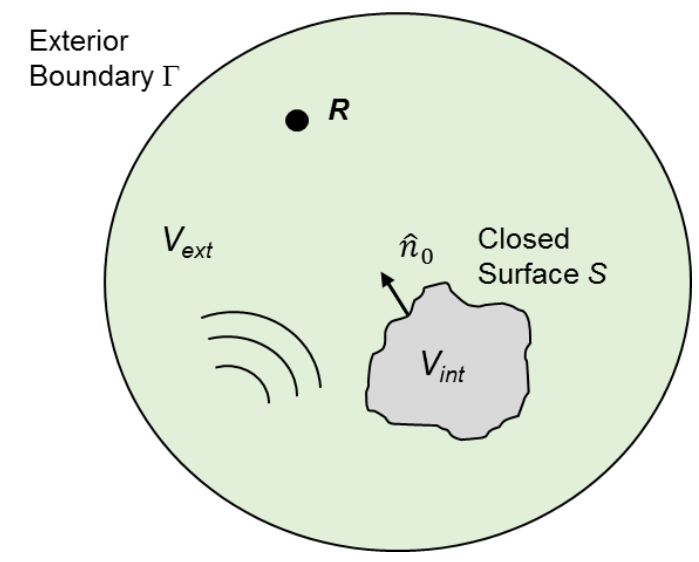

\section{Figure 3-1. The Exterior Problem Given by the Helmholtz equation}

Acoustic-structure coupling is achieved when the modes of the fluid medium excite the elastic modes of the structure. These fluid and structural modes have distinct eigenvalues and the degree of coupling between them varies based on the geometric boundary conditions of the problem, method of excitation, and frequency bandwidth considered. The finite element solution to the structural dynamic equations of motion in the structural domain consists of a system of simultaneous equations relating the displacements of the nodes to the nodal forces, while the boundary element solution for the fluid domain produces a system of equations relating the nodal displacements and nodal tractions [30]. The fundamental equations of motion for the elastic structure used in the finite element solution take the form: 


$$
[\boldsymbol{M}]\{\ddot{x}\}+[\boldsymbol{C}]\{\dot{x}\}+[\boldsymbol{K}]\{x\}=[\boldsymbol{F}]
$$

where $\boldsymbol{M}, \boldsymbol{C}$, and $\boldsymbol{K}$ are the structural mass, damping, and stiffness matrices, respectively, $\boldsymbol{F}$ is a vector representing some external forcing function, and $\{x\}$ is a vector representing the structural displacements. To reflect the FEM-BEM structural-acoustic coupling, equation 3-6 is rewritten in terms of generalized coordinates and a coupling term is added:

$$
[\boldsymbol{M}]\{\ddot{q}\}+[\boldsymbol{C}]\{\dot{q}\}+[\boldsymbol{K}]\{q\}+[\boldsymbol{L}]\left\{p_{\text {inc }}\right\}=[\boldsymbol{F}]
$$

where $\boldsymbol{M}, \boldsymbol{C}$, and $\boldsymbol{K}$ represent the respective generalized structural mass, damping, and stiffness matrices and $\boldsymbol{F}$ remains the general external forcing function vector. In the added coupling term, $\boldsymbol{L}$ is the global acoustic-structure coupling matrix of size $M \times N$, where $M$ is the number of FE degrees of freedom and $N$ is the number of BE nodes on the coupled boundary, and $\left\{p_{\text {inc }}\right\}$ is the incident pressure loading on the structure. The global coupling matrix $\boldsymbol{L}$ uses a series of local transformation matrices to relate the acoustic fluid pressure acting on boundary elements on the fluid-structure interface to nodal forces on the finite elements of the structure. The normal fluid velocities on boundary element nodes and associated normal translational displacements of the adjacent finite element nodes are related by a similar transformation matrix which differs slightly from that presented in [9] because the finite elements and boundary elements are of distinct size and the nodes are not coincident. With this coupling in place, the FE mode shapes are projected onto the $\mathrm{BE}$ mesh and displacements corresponding to the incident pressure loading from the fluid are transferred onto the FE structure; the interpolation that follows permits calculation of sound intensity and pressure at any point in the fluid domain and the nodal forces and elemental stresses at any point in the FE structure. 


\subsection{Methodology}

The starting point for the acoustic analysis performed is a representative finite element model (FEM) for the test structure to be analyzed - this structural model provides the foundation that the acoustic model is built upon. In this work, a FEM of the spacecraft mockup that had been modal test-correlated up to $100 \mathrm{~Hz}$ was used.

A normal modes analysis was conducted on the spacecraft FEM based on the acoustic analysis frequency of interest. In the final coupled acoustic FEM-BEM solution, analysis is typically conducted for frequencies up to a few hundred $\mathrm{Hz}$, where the modal density (number of modes in a given frequency band) of the structure is low enough that a BEM solution is efficient. At some threshold, the modal density of the structure will render a BEM solution inefficient and statistical methods such as SEA would need to be employed. Because multiple structural modes can contribute to a single mode in the acoustic domain, the FE normal modes analysis is conducted for a frequency range outside of the BEM analysis bandwidth to sufficiently characterize the dynamics of the structure such that the modal effective mass approaches $100 \%$. In this work all coupled BEM analyses were conducted over a frequency range of 20 to $400 \mathrm{~Hz}$ and the normal structural modes from the FEM were recovered from 0 to $500 \mathrm{~Hz}$.

\subsubsection{FE and BE Models}

The correlated spacecraft FEM shown in Figure 3-2(a) was created using commercially available FEMAP software, a pre and post-processing platform for finite element models. Boundary conditions are applied to the FEM consistent with the acoustic test configuration; in this case, a fixed-base boundary condition with constraints on all six degrees of freedom is applied at the base 
of the adapter ring where the test article is secured to the dolly. The fixed-base modes analysis was performed using the NX NASTRAN finite element solver.

While the modal analysis is performed for the entire spacecraft model, only the parts of the structure where the response output is desired are imported into the acoustic model. In this work, the region of interest is confined to the $+Y$ side solar array panels and the adjacent spacecraft bus panel. The only FE structures with significant modal contributions to this region are the $+/-$ Y Bus Panels and the two $+Y$ Solar Array panels shown in Figure 3-2(b). In the modal analysis, displacements were only recovered for nodes on these structures; these nodal displacements are used to project the structural mode shapes onto the BE mesh.

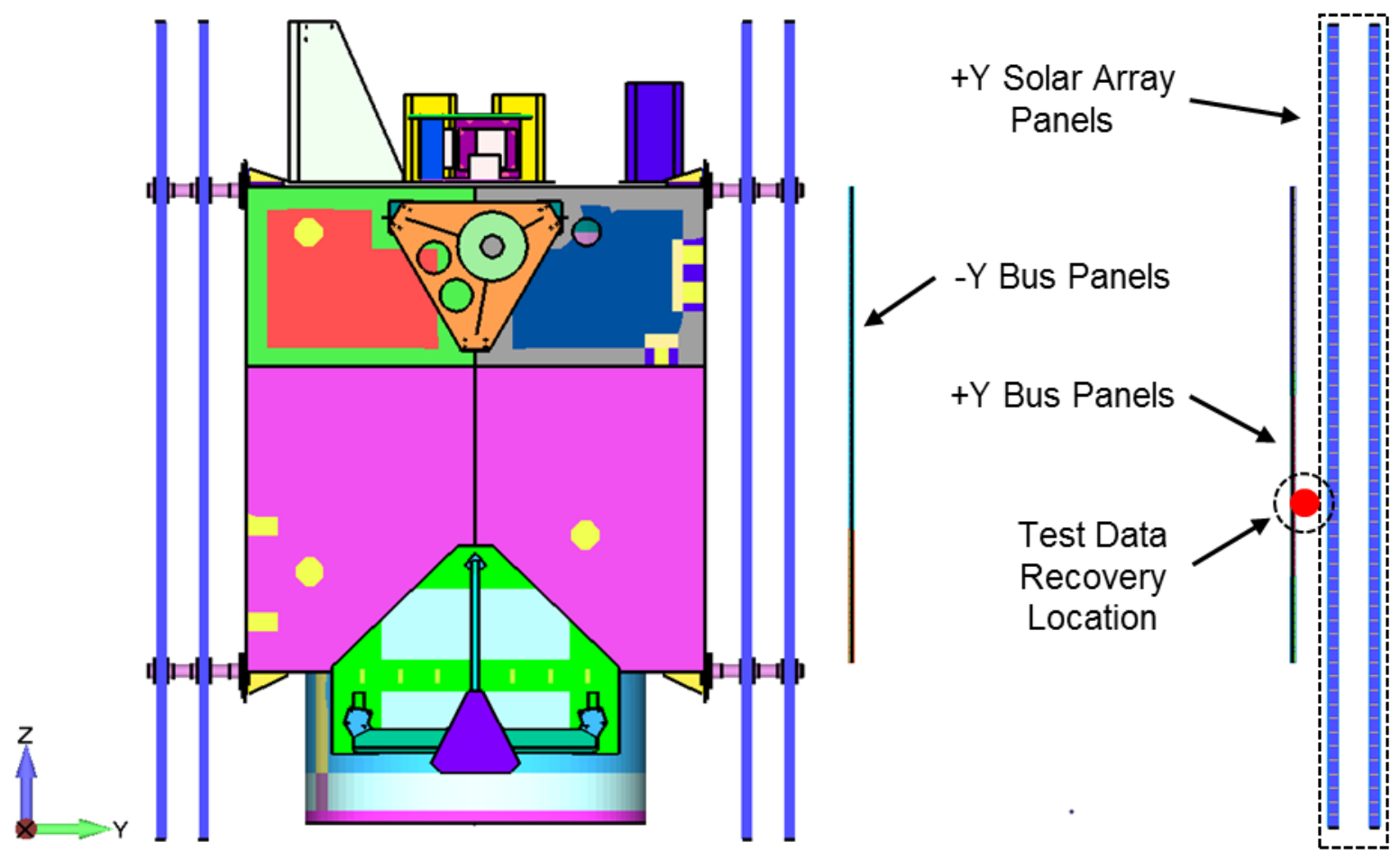

Figure 3-2. (a) Spacecraft Mockup FEM and (b) Mesh extracted for the coupled FEMBEM acoustic model 


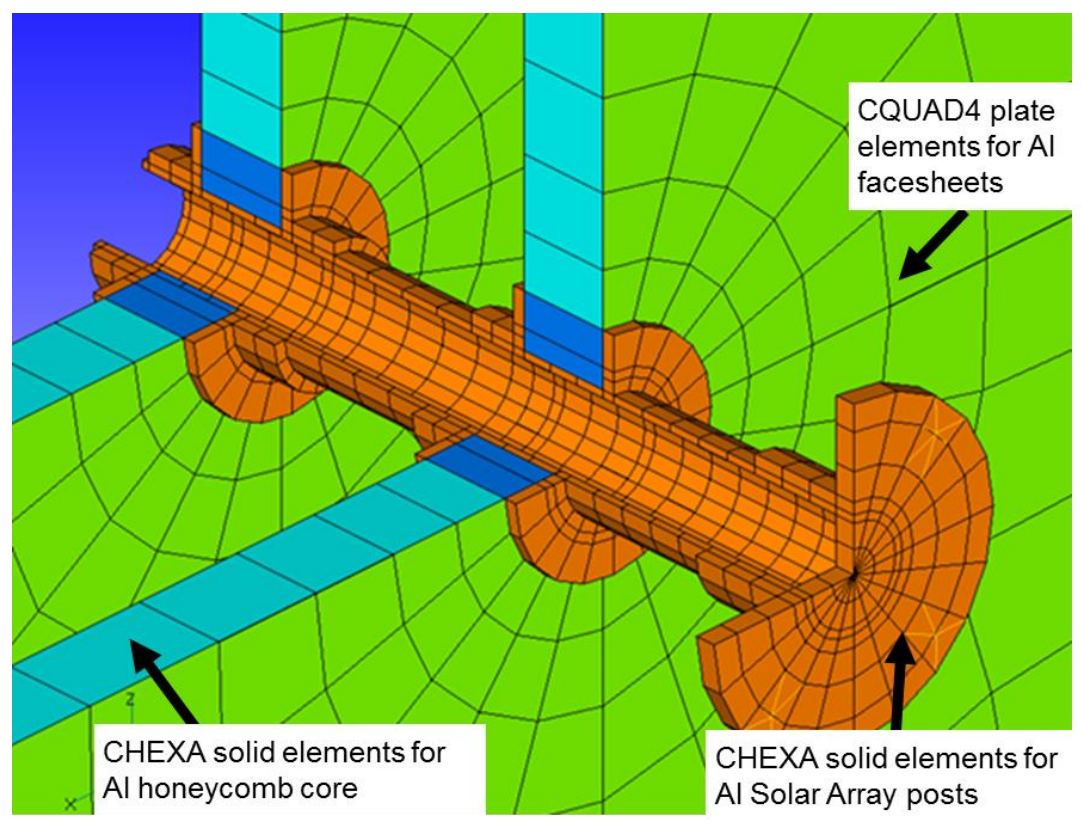

Figure 3-3. Cutaway view of Solar Array Panel Mesh in the Spacecraft Mockup FEM

Commercially available VA One software was used to create the BE model and perform the coupled FEM-BEM solution. The reader is referred to Appendix A for details on the modeling process in VA One. To create the acoustic model, a .DAT file containing the FEM is imported into VA One. The spacecraft FEM is a large model consisting of several NASTRAN element types (CONM2, RBE2, CELAS2, CQUAD4, etc.), but only mesh necessary for capturing the dynamic structural behavior of interest is needed for projecting mode shapes. In this research, the structural behavior of interest is the random vibration response at different locations on the solar array panel facesheets, so NASTRAN CQUAD4 shell elements representing these facesheets are retained.

The spacecraft mockup used in the acoustic test is constructed to dimensions similar to heritage spacecraft designs, but it does not contain the same componentry and hardware as the spacecraft it resembles. The mockup includes no cameras or fuel tanks or batteries; these components are represented by a series of mass simulators intended to capture significant mass properties. Aluminum honeycomb panels with metallic facesheets are used in place of actual solar panels. In this work, the terms "solar array" and "solar panel" with regards to the test article refer to these 
metallic honeycomb panels and not the conventional lightweight structures found on a flight model spacecraft. In the FEM, these honeycomb panels are modeled as a combination of CHEXA solid and CQUAD4 shell elements as shown in Figure 3-3: solid elements to capture the equivalent orthotropic properties of the honeycomb core and plate elements to capture the isotropic properties of the aluminum facesheets. The solid element mesh representing the core is one element thick and all panel nodes are coincident with the facesheets. The facesheet plate meshes are brought into the acoustic model to transfer the structural eigenvalues and mode shapes. This results in two sets of plate elements for each Solar Array panel, one set for each facesheet. The four aluminum posts connecting the solar arrays to the bus panels are modeled as CHEXA solid elements in the correlated FEM to capture interface stiffnesses, but these elements are not included in the coupled acoustic model. The bus panels on the spacecraft mockup are also of sandwich construction, but these are rendered in the FEM as CQUAD4 elements with composite laminate properties considering both the core and facesheets. These plate elements are also included in the coupled acoustic model to account for the structural modes of the bus. All together there are six separate meshed surfaces from the FEM included in the acoustic model consisting entirely of CQUAD4 plate elements: two representing the $+Y$ and $-Y$ bus panels and four corresponding to the facesheets of the solar array panels.

The BEM is created with its own mesh distinct from the imported FEM. In general practice, only surfaces susceptible to acoustic loads, such as lightweight panels and reflectors, are considered when creating the BE mesh and so the geometry of the BEM is simplified with respect to the FEM. Independent boundary element surfaces are generated corresponding to each of the FE panel surfaces for acoustic coupling. In order to satisfy the closed-surface boundary condition for the BE solution, additional $\mathrm{BE}$ surfaces with normal orientations along the $\mathrm{X}$ and $\mathrm{Z}$ axes are generated to 
create closed volumes for the bus and two solar array panels. The BEM consists of three enclosed six-sided regions: two corresponding to the inner and outer $+Y$ side Solar Array panels, and one box serving as a simplified representation of the Bus panels.

Figure 3-4(a) shows the FE mesh used to transfer the elastic modes from the FEM to the coupled acoustic model and (b) the corresponding BEM mesh overlaid. The BEM mesh faces shown were elastically coupled to the underlying FE mesh for surfaces with normal orientation along the Yaxis; otherwise the BEM surfaces were rigidized. Initially, FE mesh was brought into the model for the four additional Bus panels oriented in the $\mathrm{X}$ and $\mathrm{Z}$ axes to create elastic coupling on all six sides of the Bus, but it was observed that the corresponding "normal" modes for these additional nodes did not contribute to either the sound pressure levels or structural responses at the solar array panels since their normal displacements were orthogonal to those of the solar arrays. For simplification, these FE meshes and their modal contributions to the structure were omitted and the respective BE surfaces were assumed rigid. The rigid boundary condition imposed on a BEM surface specifies a normal velocity constraint on all included nodes. 

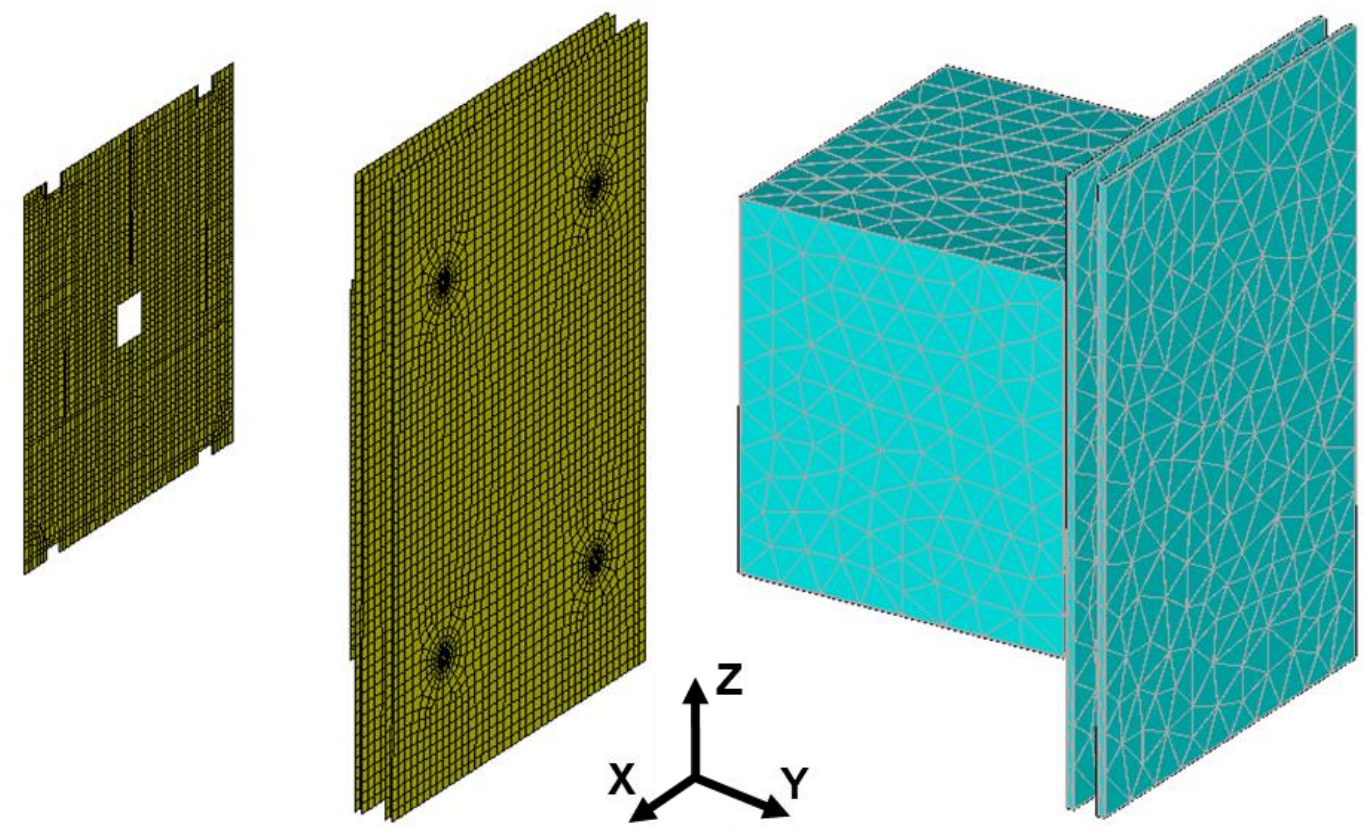

Figure 3-4. (a) FE Mesh used to transfer structural to the acoustic model and (b) overlaid BE Mesh used to define acoustic boundaries

It can easily seem from this figure that the BE mesh is much coarser than the coupled FE mesh. The reason for this is that unlike the FE mesh that is sized based on a number of considerations, the fidelity of the BE mesh is only dependent upon the BEM analysis frequency bandwidth and a selected number of elements per wavelength. The BE mesh is sized corresponding to the wavelength $\lambda$ of the incident sound waves at the maximum analysis frequency given by the relation

$$
\lambda=\frac{c}{f * n}
$$

where $c$ is the speed of sound in air at room temperature, assumed to be $13504 \mathrm{in} / \mathrm{s}$ and $n$ is the number of elements per wavelength. For a maximum BEM analysis frequency of $400 \mathrm{~Hz}$, and allocation of $n=6$ elements per wavelength, the corresponding mesh size is 5.6". Based on this calculation, the BE mesh size for all acoustic models was set to 6" unless otherwise noted. The size of the higher fidelity FE mesh varies considerably across the FEM, but does not exceed 1" for the structures considered here. 


\subsubsection{Boundary Conditions}

The boundary conditions of the BEM surfaces are all defined such that they satisfy the governing Helmholtz integral equation. Each BE surface (called FE Faces in VA One) has a defined "Front" and "Back" face that can receive excitation from plane waves in the acoustic medium. For consistency, the "Front" faces of all elements in the BEM mesh are oriented outwards as shown in Figure 3-5 and these faces are subject to acoustic excitation such that all exterior surfaces receive excitation.

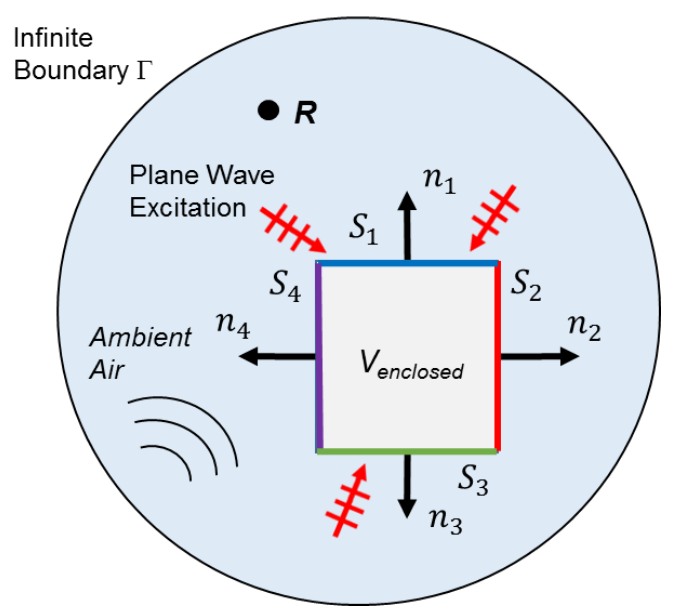

Figure 3-5. Enclosed BE Representation of Spacecraft Structure

\subsubsection{Acoustic Excitation}

After the elastic coupling between the FE structures and BE surfaces is established and the boundary conditions for the fluid domain are defined, the acoustic excitation is created. An input spectrum for the sound pressure levels on $1 / 3^{\text {rd }}$ octave bands is defined based on the measured field levels in the test. In standard practice, these levels are based on the protoflight launch vehicle fairing environment, assumed to be diffuse so that this same excitation is valid everywhere. In this case a test environment is approximated that is distinct from that of the launch vehicle fairing. The average sound pressure levels at each control and monitor microphone are calculated over the time 
history from the full-level acoustic test. Since the control and monitor microphone readings are representative of the controlled acoustic field, an average of these can be used to describe the excitation on the spacecraft exterior. For the acoustic field region of interest between the bus and solar arrays, noticeable differences were observed between the measured test levels and analytical predictions at multiple frequency bands. One reason for this disparity in analytical prediction could be the input spectrum applied to the model. It can be reasoned that the local environment in this region is best approximated by some subset of the control and monitor microphone averaged SPL readings from the test, using microphones positioned closest to the solar array and excluding microphones located on the other side of the spacecraft. Using the established baseline model, a study was done to determine what group of averaged control and monitor microphone channel readings could be used to best predict the SPL between the bus and solar arrays. As expected, the best selection for input averaging was the five control/monitor microphones nearest to the corner of the spacecraft where the additional test data was recovered.

The acoustic excitation from this derived input profile is applied in the model using a set of uncorrelated plane waves at random phase. In keeping with standard practice, the acoustic field is assumed diffuse and a system of 50 equally spaced plane waves was generated in spherical coordinates at $45^{\circ}$ radial increments. To assess the effectiveness of this plane wave distribution in creating a diffuse field environment, the number of plane waves was increased to 200, with a radial distance of $20^{\circ}$ between them. The sound pressure levels at different points in the model were compared for both plane wave allotments, but the results were quite consistent; this indicates that a selection of 50 plane waves is sufficient for the diffuse field assumption. It should be noted that increasing the number of plane waves in the model significantly increased the CPU time for the BEM solution, so an appropriate choice of plane waves is important. By the diffuse field 
assumption, all $\mathrm{BE}$ surfaces were subject to the same excitation. Although this is not necessarily the case for DFAT, the acoustic environment of interest is between two closely-spaced relatively stiff panels and it can be assumed that there is enough local diffraction of sound energy between them to create a nearly diffuse field condition.

\subsubsection{Damping}

Assumptions for the damping in the acoustic field and spacecraft structure can be specified for the acoustic analysis in multiple places. In the modal analysis of the test-correlated FEM, the damping of the air between solar array panels is accounted for using the NASTRAN Virtual Fluid Mass parameter MFLUID. A Damping Loss Factor (DLF) equivalent to twice the damping ratio is assigned to all FE structures in the coupled acoustic model. A baseline value of 0.04 or a $4 \%$ loss factor was assumed for all FE structures across the analysis bandwidth; this is a standard value that has been assumed for similar analyses [25] and is selected based on the comparison of structural response prediction with test data for different damping values. The effect of damping loss factor on structural response is most clearly observed at narrow band response peaks. A separate loss factor can be specified for the air in the fluid domain for the intermediate BEM solution, but this is assumed to be zero since the fluid mass is already accounted for in the FE normal modes analysis.

\subsubsection{Solution and Data Recovery}

With elastic coupling between FE and BE models established, boundary conditions defined, and acoustic excitation imposed, the intermediate BEM solution can be calculated. The BEM solution is performed on $1 / 42^{\text {nd }}$ octave bands in order to discretize the analysis frequency bandwidth for good modal resolution - this produces 189 discrete frequency bands on the 20 to $400 \mathrm{~Hz}$ 
bandwidth. Evaluation of the fully-populated spectral density matrices is performed on each $1 / 42^{\text {nd }}$ octave band, such that the fluid velocities at all nodes and the incident pressures on all boundary elements are calculated. Depending on the model fidelity, this solution can be very time-intensive, but the average CPU time for the baseline model was under 25 minutes.

Next, the final coupled FEM-BEM solution is performed over the same $1 / 42^{\text {nd }}$ octave bands as the intermediate $\mathrm{BE}$ solution that interpolates $\mathrm{BE}$ nodal velocities and elemental pressures at the acoustic modes with FE nodal displacements at each of the FE modes. Table 3-1 summarizes the parameters used in the solution of the baseline model.

Table 3-1. Baseline Acoustic Model Solution Parameters

\begin{tabular}{|c|c|c|}
\hline Parameter & Value & Unit \\
\hline $\begin{array}{c}\text { FEM Normal Modes } \\
\text { Analysis Frequency Range }\end{array}$ & $0-500$ & $\mathrm{~Hz}$ \\
\hline $\begin{array}{c}\text { BEM Analysis Frequency } \\
\text { Range }\end{array}$ & $20-400$ & $\mathrm{~Hz}$ \\
\hline $\begin{array}{c}\text { Solution Frequency } \\
\text { Bandwidth in Octaves }\end{array}$ & $1 / 42$ & $\mathrm{~Hz}$ \\
\hline FEM Mesh Size (approx.) & 1 & in \\
\hline BEM Mesh Size (approx.) & 6 & in \\
\hline $\begin{array}{c}\text { Structural Damping Loss } \\
\text { Factor (DLF) }\end{array}$ & 0.04 & N/A \\
\hline
\end{tabular}

Responses are recovered from the acoustic model solution at discrete locations in two forms. The random vibration response specified as acceleration spectral density in units of $\mathrm{g}^{2} / \mathrm{Hz}$ is measured at specific nodes in the FE mesh and the Sound Pressure Level in $\mathrm{dB}$ is measured at specific points in the acoustic domain. The structural responses are typically recovered on FE nodes corresponding to the accelerometer locations in the test for direct comparison. In this work, structural responses were measured at five nodes on the exterior (+Y side) of Panel 2 and at six 
nodes on the inside (-Y side) of Panel 1 as shown in Figure 3-6; the recovery node on Panel 1 corresponding to the test accelerometer location is highlighted. Similarly, Figure 3-7 displays the discrete locations where the SPL in the acoustic field was measured in Regions 1 and 2 at the midpoint between adjacent panels. The recovery point corresponding to the microphone placed in Region 1 during the acoustic test is highlighted. Results obtained at these recovery points are used for comparison in the subsequent trade study. 


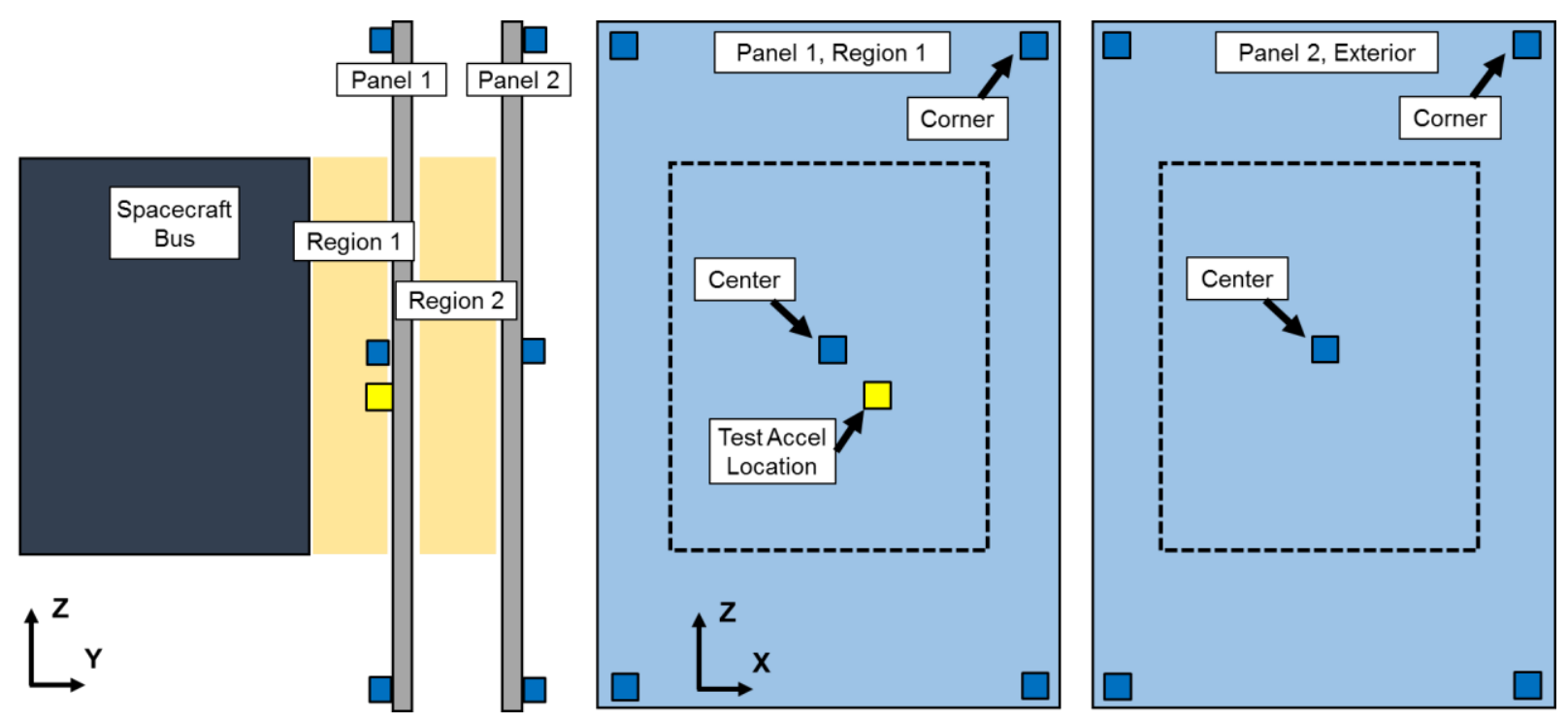

Figure 3-6. Analytical Structural Response Measurement Locations

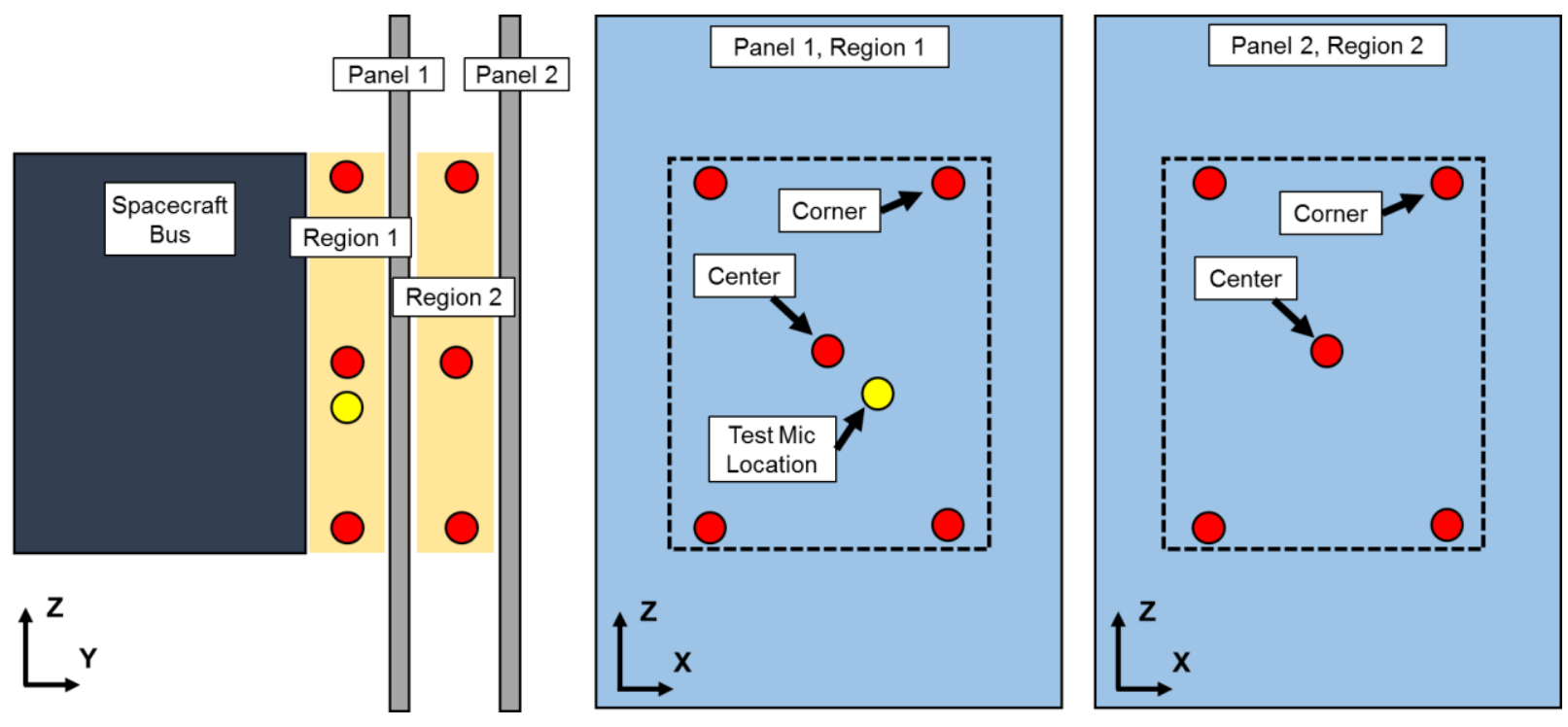

Figure 3-7. Analytical SPL Recovery Locations 


\section{Trade Study}

A trade study was conducted to observe how the acoustic environment between the spacecraft bus and the stowed array panels and the associated structural responses of the array panels changed with respect to varied panel spacing, damping assumptions, and BEM mesh fidelity. This chapter presents the motivation for this trade study, the parameters of interest, and the corresponding trades performed. Validation for the modeling effort is given. Key findings from the trades performed are presented here and discussed. Where possible, the results are compared with test data.

\subsection{Definitions and Parameters of Interest}

The trade study was conducted in order to assess the sensitivity of analytical results to changes in fundamental modeling parameters and understand what level of model fidelity best approximates the physical test configuration. Additionally, there is interest in observing the sensitivity of the model response measurements to changes in the size of the acoustic cavities between panels. The primary parameter of interest in this study is the distance between the spacecraft bus and the solar array panels, $d_{1}$. This dimension on the test article is four inches, which is much smaller than the overall panel dimensions. In an acoustic test environment, this creates a cavity region that sees significantly higher sound pressure levels than the ambient acoustic field around the spacecraft on most frequency bands. This result is expected for enclosed regions, where acoustic energy is refracted several times between structural elements and it is of interest to see how well the conventional analysis method previously defined can predict these higher levels. It is also informative to measure the dynamic responses on structures in this region, since a change in the adjacent sound pressure level will directly impact the pressure loading and associated nodal velocities on the structure. These two analytical measurements are coupled because improvements 
in the SPL prediction in the cavity will result in a more realistic acoustic excitation on the adjacent structure.

For a given acoustic excitation spectrum, one would expect that as $d_{1}$ increases, the SPL in Region 1 will converge to the free-field levels equivalent to the input spectrum. This trade study investigates how the SPL measured in the cavity varies with incremental increases in $d_{1}$ over the baseline value. The associated variations in the structural responses at different points on Panels 1 and 2 are also studied. A similar investigation is performed by varying the distance $\mathrm{d}_{2}$ between Panels 1 and 2 for each value of $d_{1}$ to understand response contributions from adjacent volumes. This structural configuration of closely stacked panels is often encountered in spacecraft designs. When satellites are secured in their stowed position inside the launch vehicle fairing, the solar arrays are folded in and attached to the bus payload panels at discrete points. There is inevitably some cavity environment created in this shallow volume between the bus and folded arrays that has the potential to generate local peaks in the acoustic field levels; the size of this volume will depend on the relative dimensions of the bus and the adjacent solar array and the distance between panels can vary based on the hinge attachment mechanism and clearance requirements. For these cavity environments, it is important to analytically predict the frequencies at which SPL peaks will occur and how they might impact the dynamic responses of lightweight structures located nearby.

From a modeling standpoint, there are a number of factors that can contribute to accurate prediction of the acoustic environment. In the FEM, correlation of the structural modes to modal testing data will provide good approximation for the structural dynamics in the acoustic model. Damping assumptions are made to control the amplitudes of narrow band responses and the structural response data from the test is compared with analytical predictions for some different DLFs assigned over the entire frequency spectrum. In the BEM, accurate prediction of the sound pressure 
level between structural elements depends on accurate representation of the structural geometry in terms of panel size and thickness and the clearance between them. The BE mesh must be appropriately sized for a few different reasons. The incident wavelength of any plane wave decreases linearly with increased frequency, which means that as the frequency analysis bandwidth increases, the minimum size of the boundary elements necessary to capture the excitation of incident sound waves is reduced; this translates to a higher-fidelity mesh. At higher frequencies, the structural mode shapes become much more complicated and exhibit an increasing number of inflection points, so higher fidelity BE meshes are required to accurately capture modal projections from the FE nodal displacements. Lastly, the CPU time for the intermediate BEM solution varies exponentially with the number of $\mathrm{BE}$ nodes so that using an unnecessarily refined $\mathrm{BE}$ mesh can be computationally costly with meager improvements in modeling accuracy. To confirm this, a mesh convergence study was performed on the baseline model by varying the BE mesh size in increments of 2" and comparing trends in the CPU time with the analysis predictions.

The trade study performed in this work is mapped out in Figure 4-1. The distance $d_{1}$ between the Bus panel and Panel 1 was increased from the baseline distance of 4" in 50\% (2") increments up to 12 ". For each of these five $\mathrm{d}_{1}$ values, $\mathrm{d}_{2}$ was also incremented in $50 \%$ steps from 3.75 " to 11.25 ". All of these combinations represent a total of 25 different models. Using the baseline values for $\mathrm{d}_{1}$ and $\mathrm{d}_{2}$, the DLF was traded using values of $1 \%, 2 \%$, and $3 \%$; these DLF values were applied to all FE mesh in the acoustic model across the entire analysis frequency spectrum. Lastly, the baseline model was solved using different BEM mesh sizes from 2" to 10" in 2" increments. Including the baseline model, this trade study reflects results from 32 separate models. 


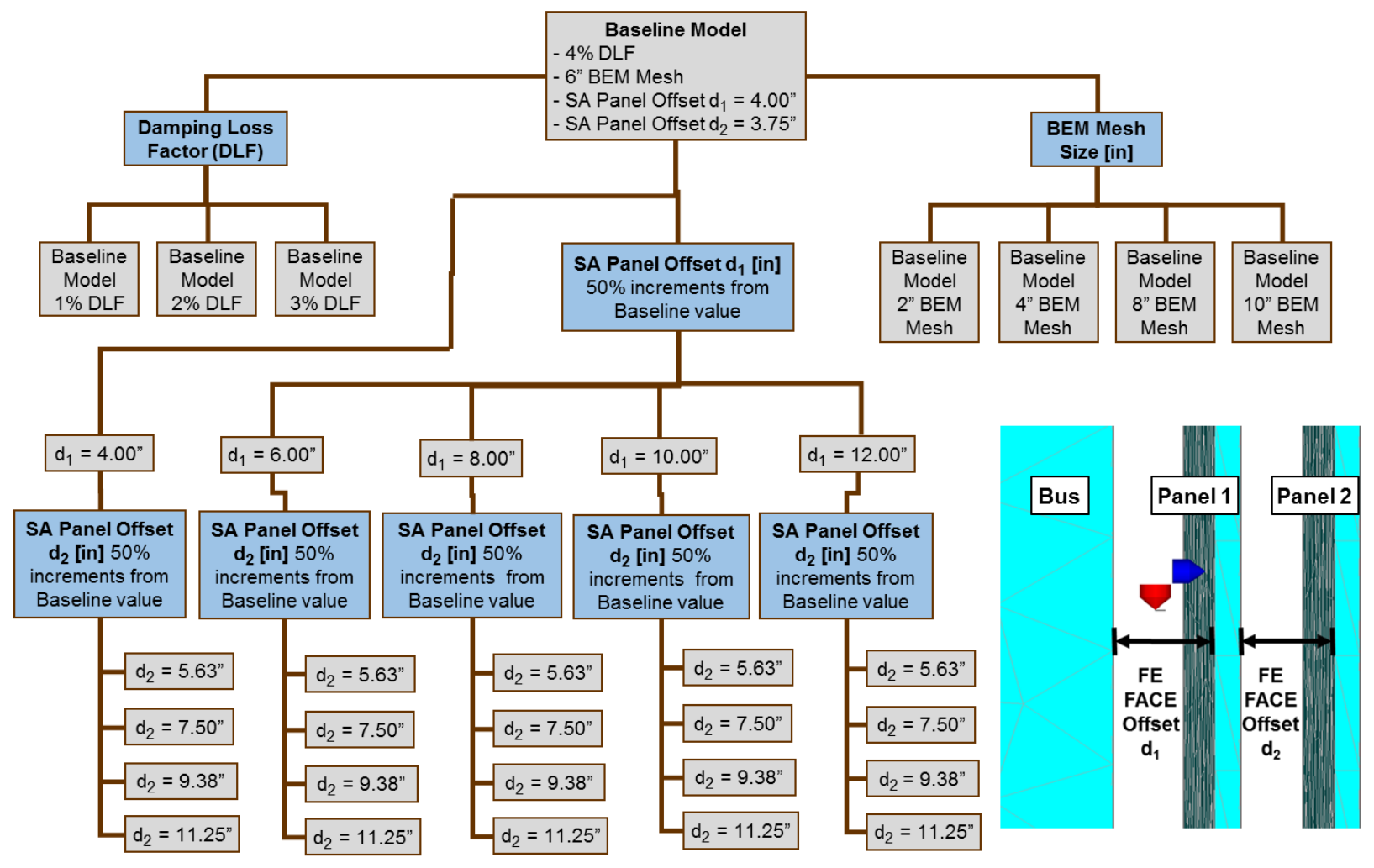

Figure 4-1. Trade Study Map

\subsection{Baseline Results}

Figure 4-2 shows the predicted random vibration response of the accelerometer on Panel 1 using the correlated FEM compared with the measured response levels during the acoustic test. Overall, the analytical predictions are fairly consistent with the test data, with a close approximation of the root-mean-square (RMS) acceleration across the bandwidth; the three distinct panel modes under $100 \mathrm{~Hz}$ are captured, although the first two are under-predicted by $5 \mathrm{~Hz}$. These predictions assume a 4\% DLF, which is considered conservative, but as noted later different DLF assumptions did not appreciably improve this prediction. The analytical prediction for SPL at the microphone location between the Bus and SA Panels on each of the controlled $1 / 3^{\text {rd }}$ octave frequency bands is shown in Figure 4-3. On most bands, the prediction showed less than $3 \mathrm{~dB}$ difference from the measured test levels, with a maximum under-prediction of $5 \mathrm{~dB}$ on the $200 \mathrm{~Hz}$ band. There is a consistent 
trend between predictions in the SPL in Region 1 and the adjacent response on Panel 1 showing under-prediction up to around $160 \mathrm{~Hz}$, with a transition to over-prediction at the $200 \mathrm{and} 250 \mathrm{~Hz}$ bands. It should be noted that the FE structural modes have only been correlated to $100 \mathrm{~Hz}$, where the modes were still distinguishable; above this frequency, the response predictions do not reflect any test correlation. The black line in Figure 4-3 and subsequent SPL plots represents the acoustic input spectrum applied to the boundary surfaces consistent with the diffuse field assumption. These input levels were applied to all acoustic models for this trade study.

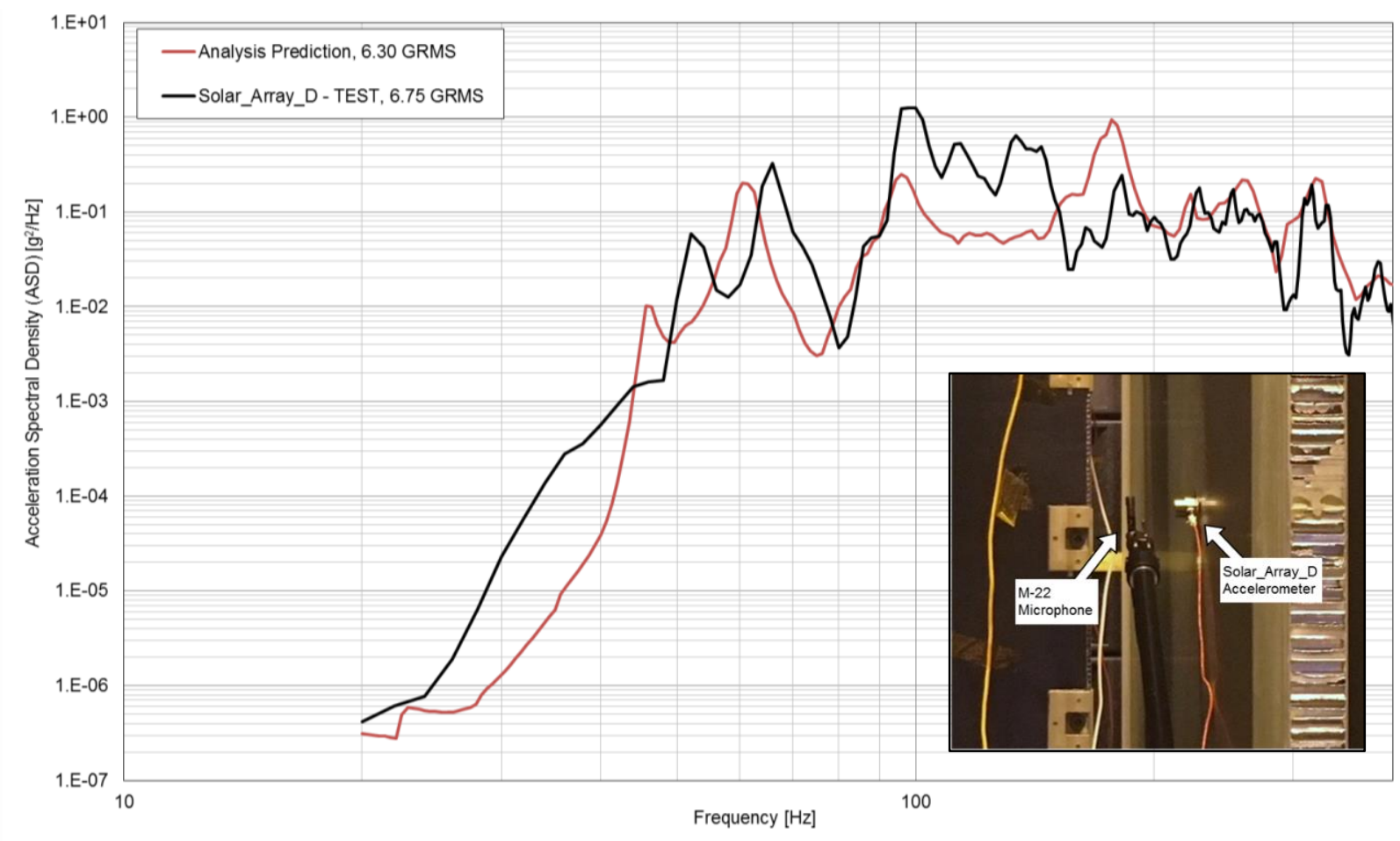

Figure 4-2. Accelerometer on SA Panel 1 next to M-22 Microphone: Comparison of Test Data to FEM-BEM Prediction 


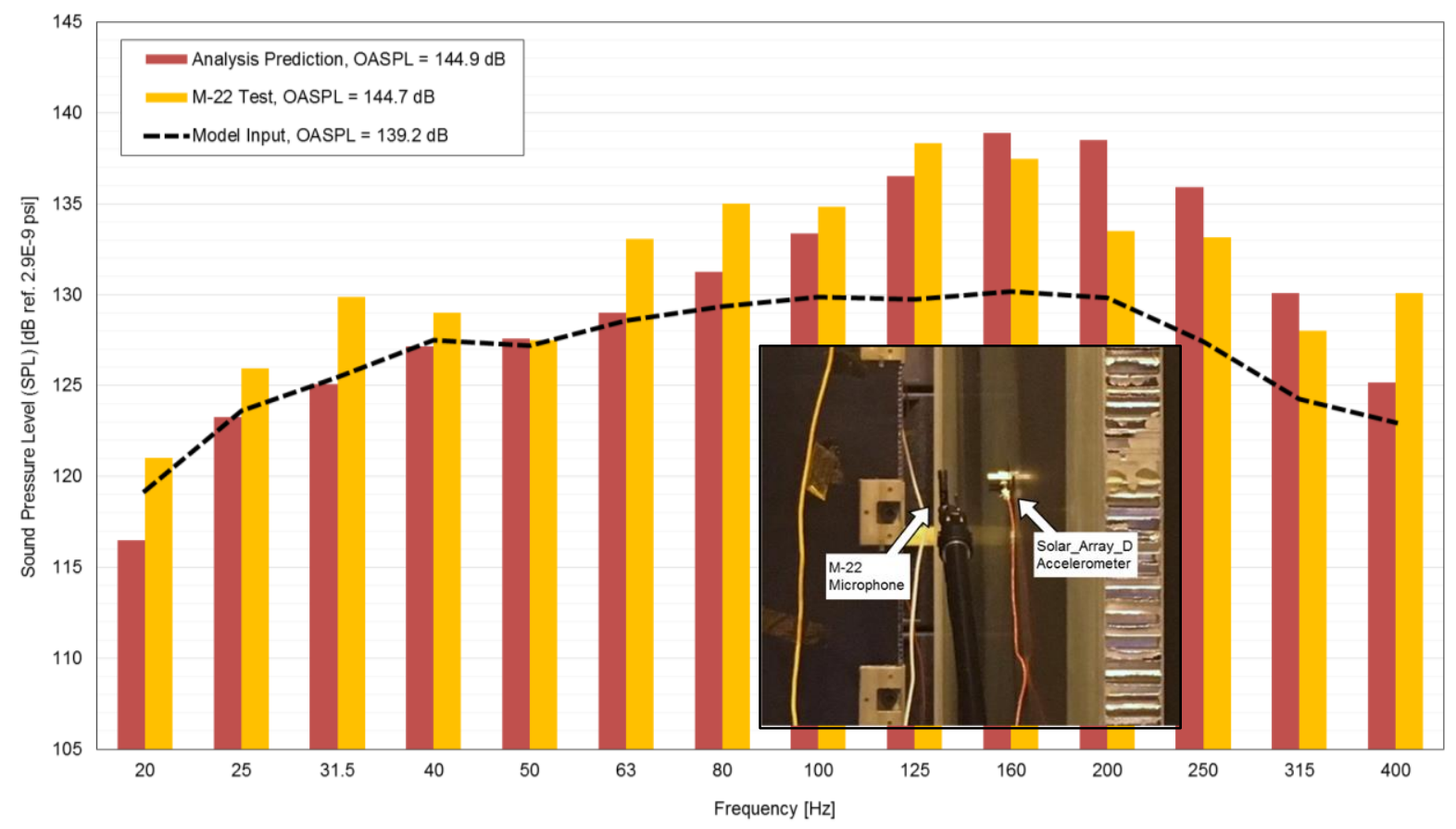

Figure 4-3. M-22 Microphone between Bus and SA Panel 1: Comparison of Test Data to FEM-BEM Prediction

Figures 4-4 and 4-5 serve as a reference in comparing the trends in response variation from one location to another when surveying results from the trades. Figure 4-4 shows the variation in ASD response predictions for different locations on Panels 1 and 2, while Figure 4-5 shows the variation in SPL predictions for different locations in Regions 1 and 2. In general, higher structural response levels were predicted for frequencies above $100 \mathrm{~Hz}$ at the center and corners of Panel 1 compared with the test accelerometer location. In addition, the SPL predictions were highest in the center of both regions, with levels decreasing along any direction moving radially outward. 


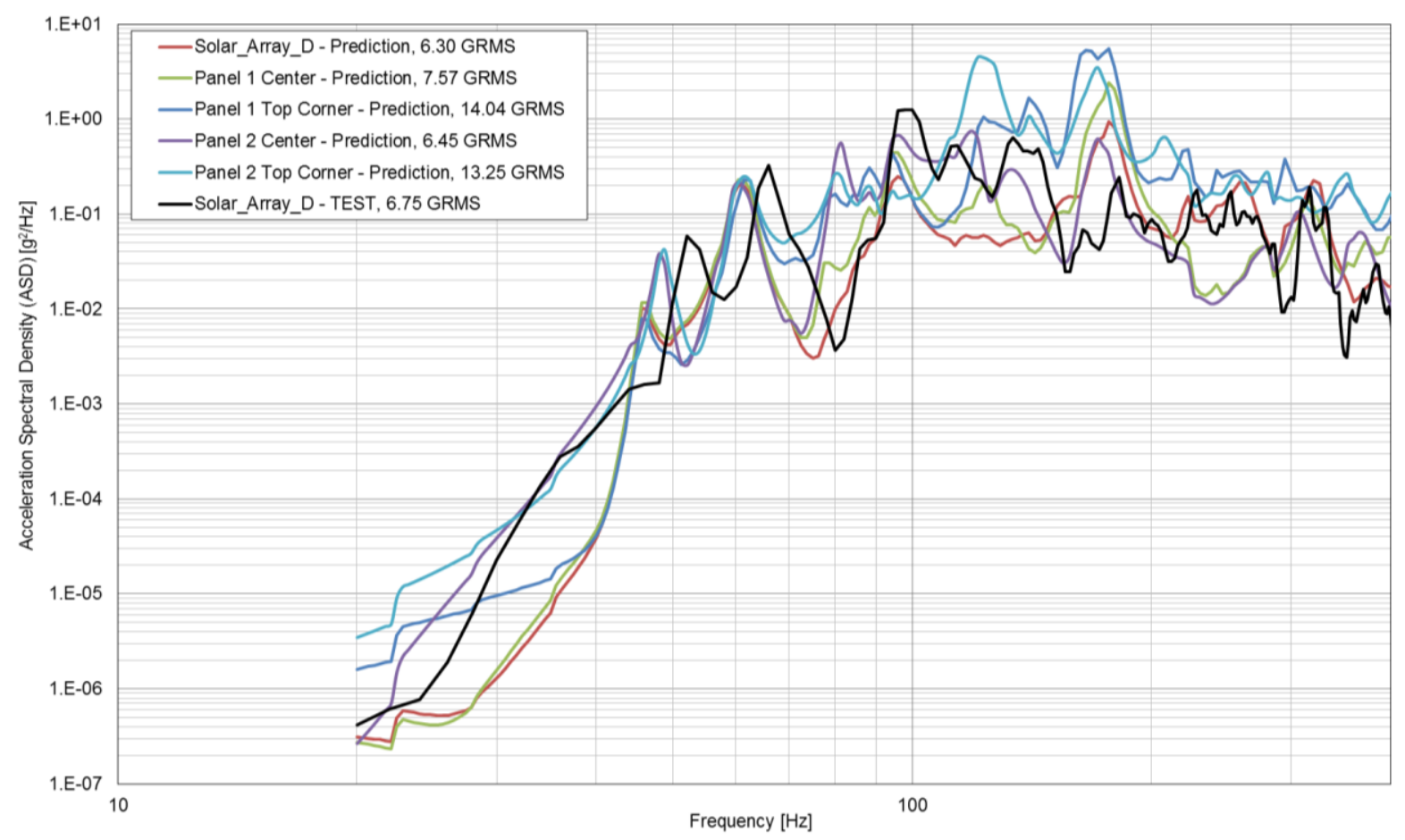

Figure 4-4. ASD Predictions for Different Locations on Panels 1 \& 2

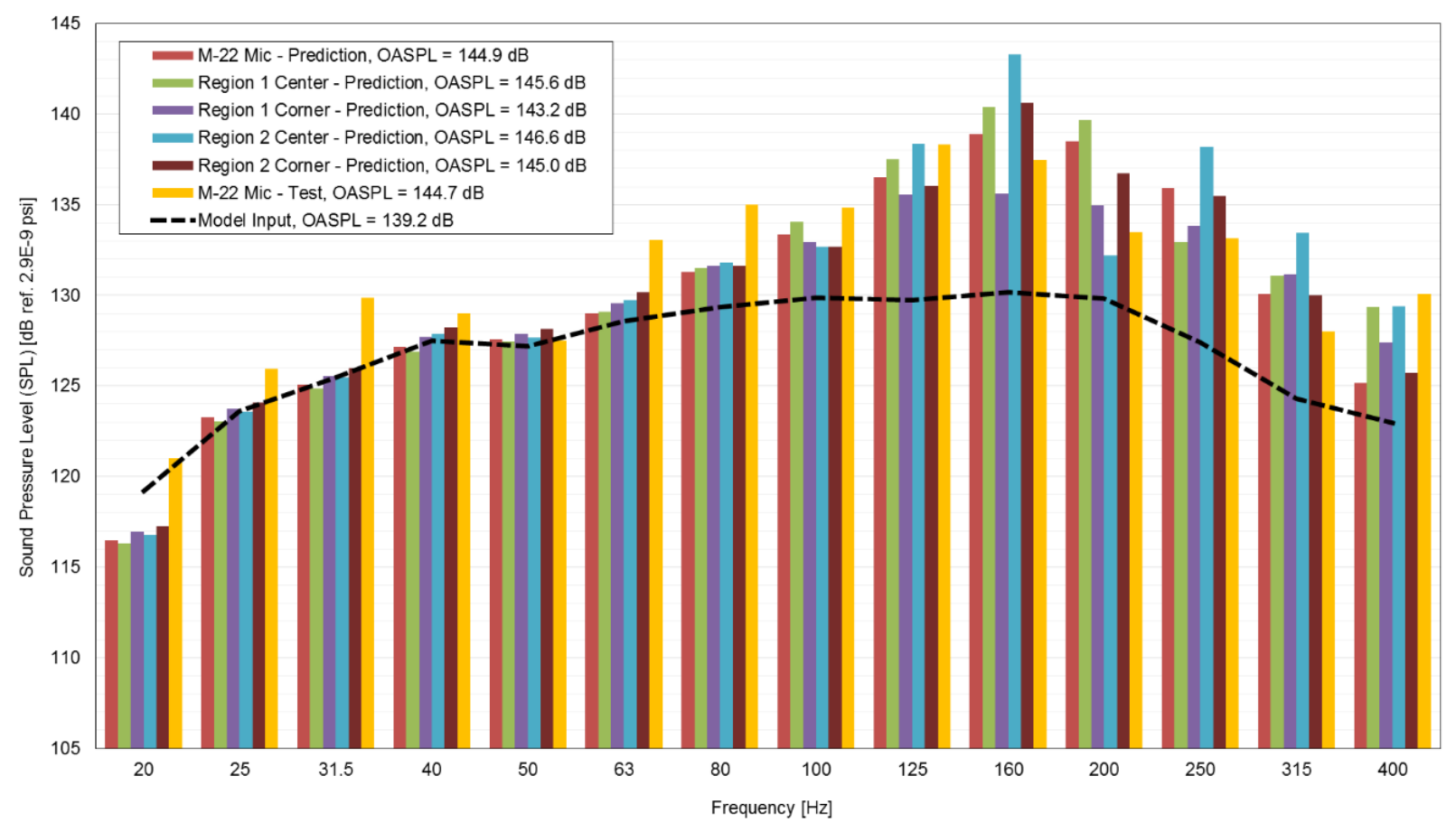

Figure 4-5. SPL Predictions for Different Locations in Regions 1 \& 2 
It is also of interest to observe any coupling that may be present between the structural modes of Panel 1 and acoustic cavity modes of Region 1 and see to what degree this coupling is represented analytically. Figure 4-6 illustrates this modal coupling by plotting the test accelerometer and microphone measurements over the same frequency bandwidth in terms of power spectral density. As previously shown in Figure 4-2, the structural response is plotted with solid lines in terms of acceleration spectral density in units of $\mathrm{g}^{2} / \mathrm{Hz}$ on the left $\mathrm{y}$-axis, while the microphone-measured sound levels are plotted with dashed lines in terms of pressure spectral density in units of $\mathrm{psi}^{2} / \mathrm{Hz}$ on the right y-axis. The energy in the system is concentrated at frequencies corresponding to local response peaks; coupling between the panel and cavity modes is represented by alignment of peaks for acceleration and pressure spectral densities. Observation of the test spectral densities shows some alignment, particularly in the 100 to $200 \mathrm{~Hz}$ range. The baseline analytical predictions presented in Figures 4-2 and 4-3 are plotted together in Figure 4-6 for comparison. These analytical predictions also show some coupling between the structural and cavity modes across the 20 to 400 $\mathrm{Hz}$ bandwidth, notably at the $60 \mathrm{~Hz}, 95 \mathrm{~Hz}, 160 \mathrm{~Hz}$, and $180 \mathrm{~Hz}$ bands. It seems that the relative distribution of energy in the system between structural and acoustic modes is fairly consistent for both test and analytical datasets, indicating that the structural modes of Panel 1 have significant impact on the cavity modes of Region 1 and vice versa. The analytical approximation to this coupling effect is shifted to lower frequencies. 


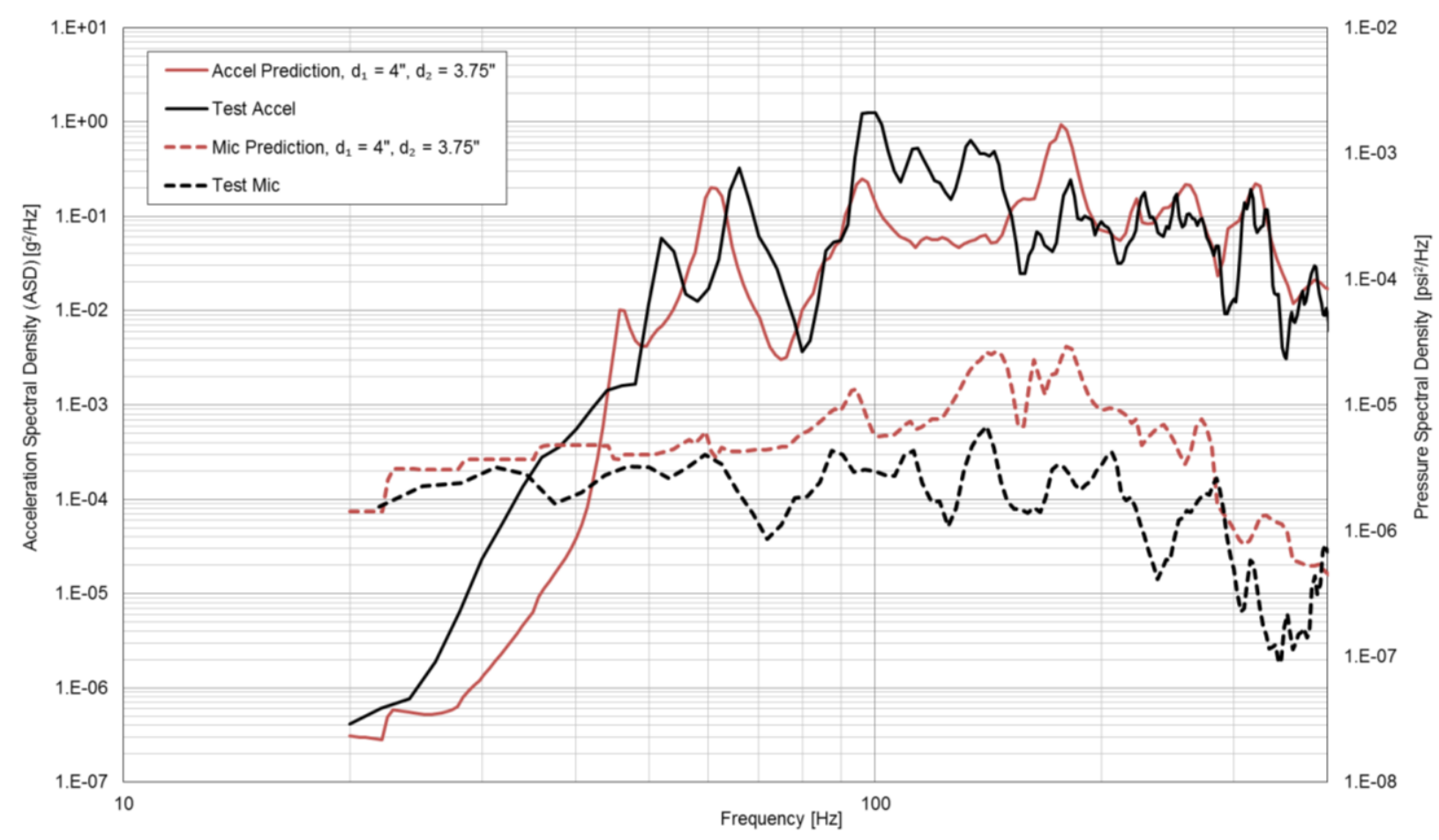

Figure 4-6. Analytical Prediction of Modal Coupling between Structural and Acoustic Modes at Test Measurement Points

\subsection{Solar Array Spacing Trades}

The first trades to be conducted involved incremental changes in the separation distance $d_{l}$ between the Bus and SA panels. This distance was increased in 50\% (2") increments over the baseline 4" distance in order to observe how the associated predicted acoustic field levels and structural responses would change. Figure 4-7 shows the ASD predictions at the test accelerometer location for this trade plotted against the test measurement. The most significant variation is seen between 100 and $200 \mathrm{~Hz}$; from 100 to $175 \mathrm{~Hz}$, the response predictions showed a steady increase with increased $d_{l}$, while predictions between 175 and $200 \mathrm{~Hz}$ showed a steady decline. The RMS acceleration converged to a value of 5.44 Gs for $d_{1}=12$ ", which indicates that further increasing $d_{l}$ would produce negligible change in the response profile. Figure 4-8 shows the shifting ASD predictions for a point on the center of Panel 1. Similar trends are seen here compared with those for the accelerometer location, although the response peaks at $95 \mathrm{~Hz}$ and $180 \mathrm{~Hz}$ are higher. The 
predicted response variation at a point on the corner of Panel 1 is depicted in Figure 4-9. In this case, the peak responses seem to be less sensitive to the changing separation distance $d_{1}$ compared with points in the middle of the panel. Responses on the exterior of Panel 2 exhibited comparably less variation for this trade, as shown in Figures 4-10 and 4-11. Small variations in the response at the center of Panel 2 were confined to the 100 to $175 \mathrm{~Hz}$ bandwidth, while response predictions at the corners showed negligible change with $d_{l}$.

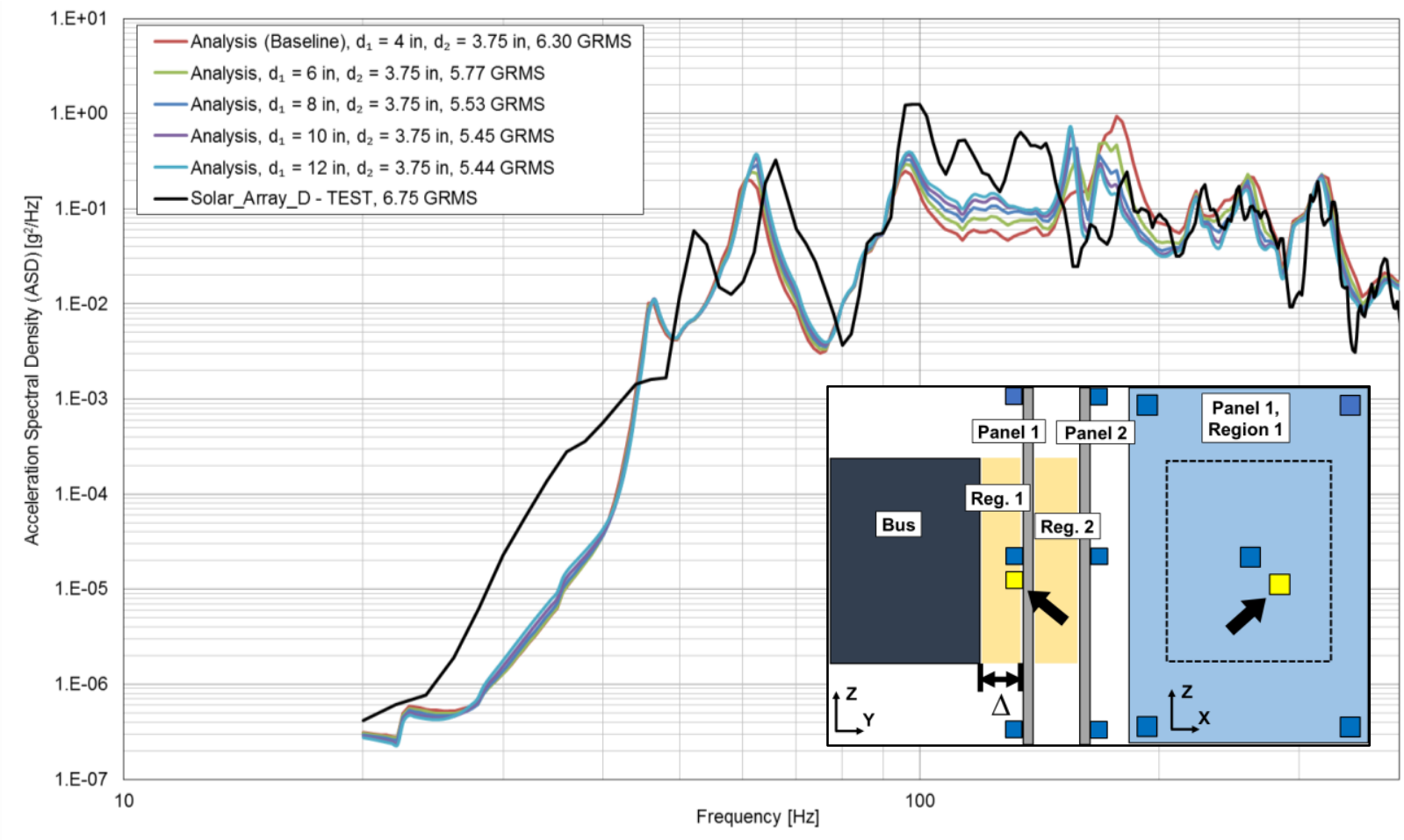

Figure 4-7. ASD Prediction at Test Accel Location for varied d1 


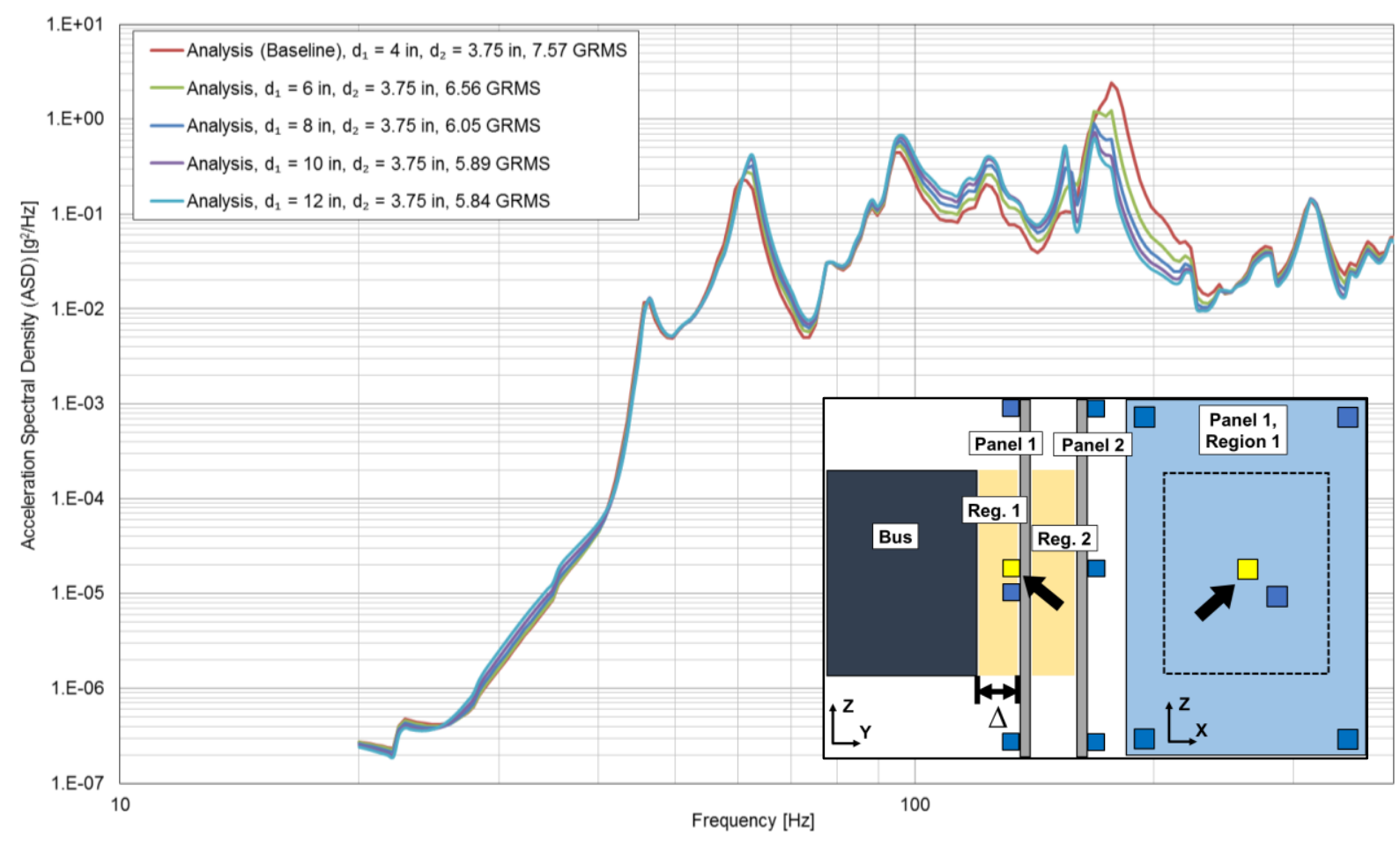

Figure 4-8. ASD Prediction at Panel 1 Center for varied $d_{1}$

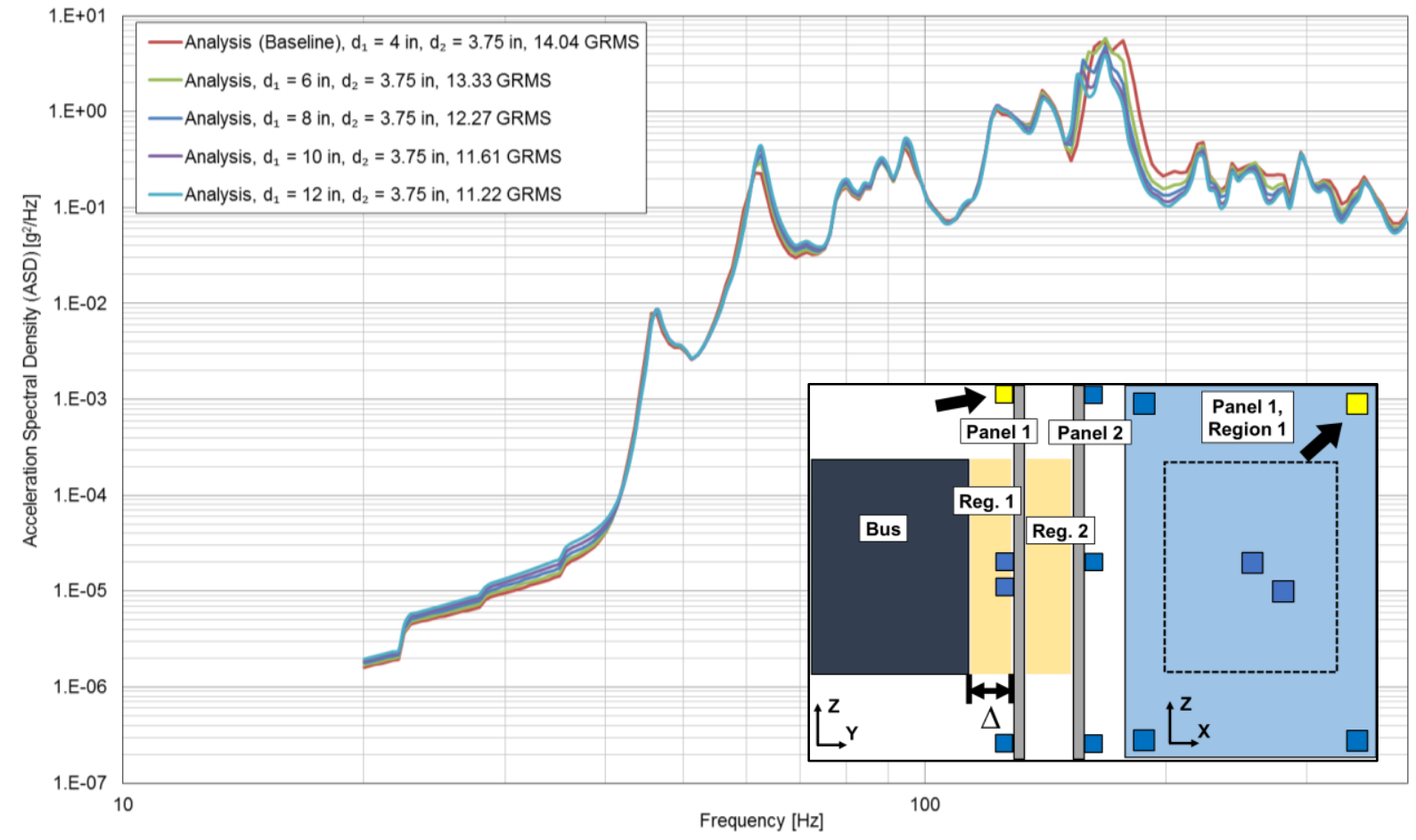

Figure 4-9. ASD Prediction at Panel 1 Top Corner for varied $d_{1}$ 


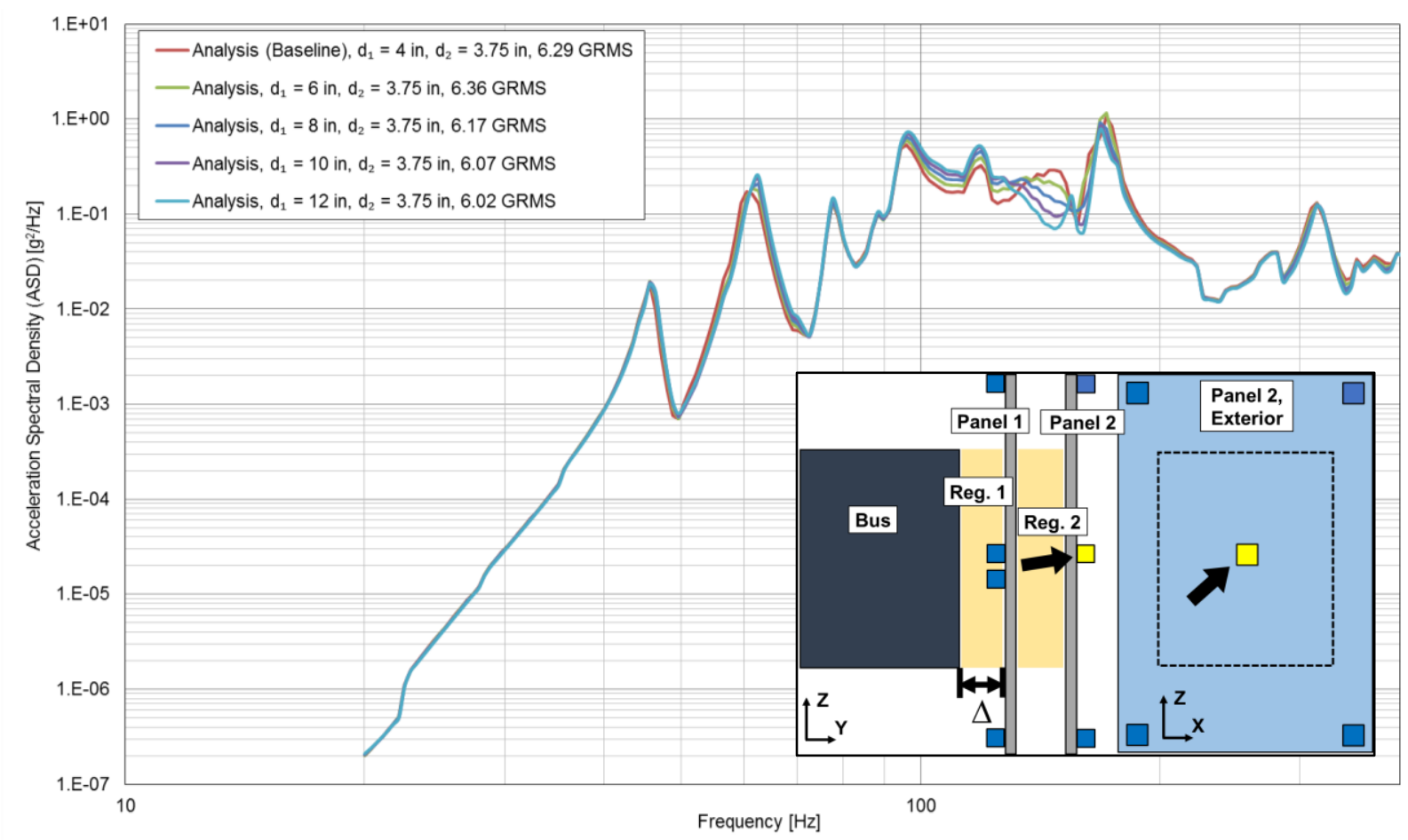

Figure 4-10. ASD Prediction at Panel 2 Center for varied d1

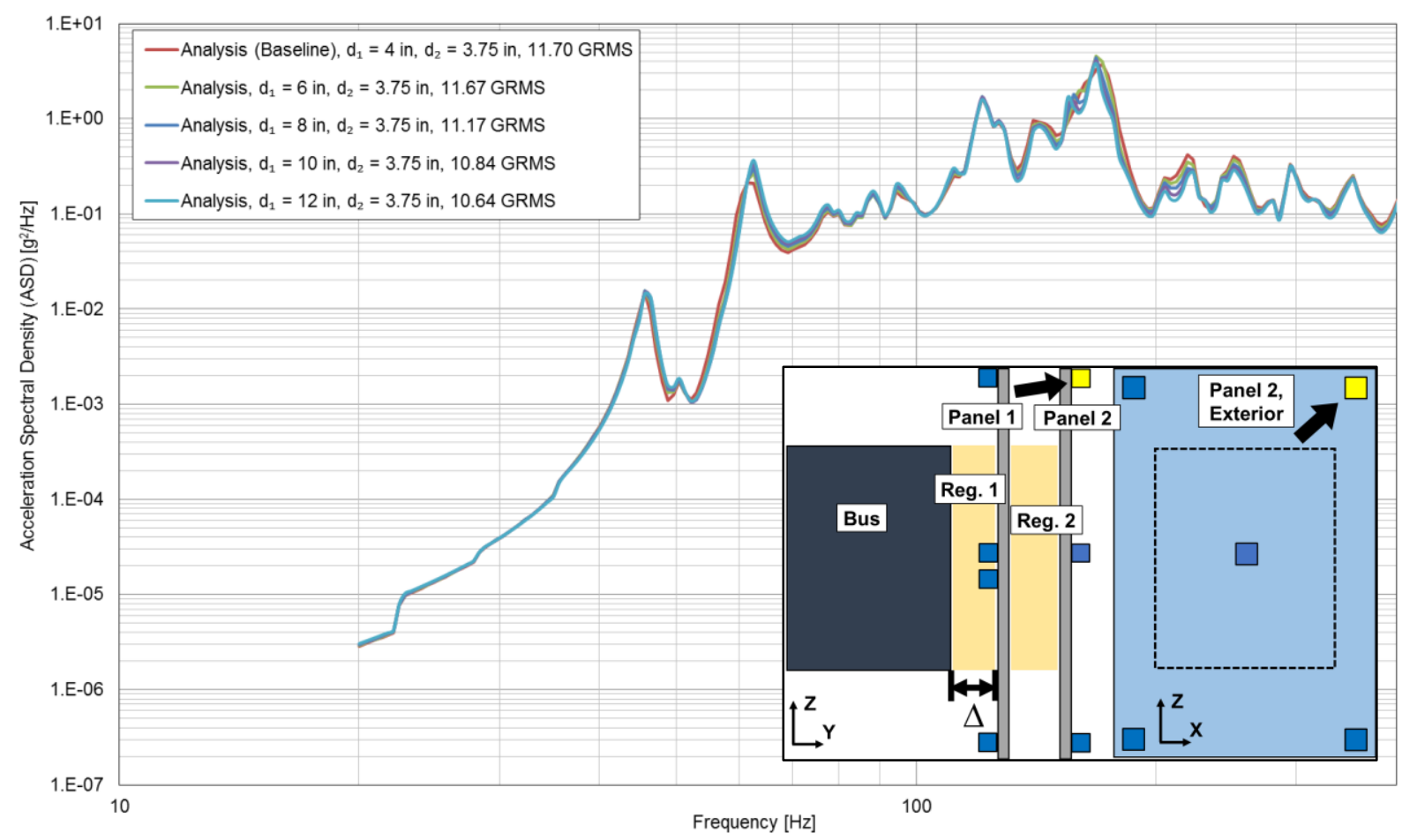

Figure 4-11. ASD Prediction at Panel 2 Top Corner for varied d1 
Figures 4-12, 4-13, and 4-14 show analytical predictions for the SPL at different points in Region 1 corresponding to varied $d_{l}$. It should be noted that as $d_{l}$ was increased, these field points were adjusted so that they were aligned in the mid-plane between adjacent panels. From the predictions at the test microphone location shown in Figure 4-12, it can be seen that the SPL converges to the test measurement on most frequency bands above $80 \mathrm{~Hz}$. While there is a consistent decrease in the OASPL (sound energy) for incremental increases in cavity volume, $d_{l}$ would need to be significantly increased beyond 12" to achieve the far-field levels in the model represented by the input curve at an OASPL of 139.2 dB. The SPL measurements at the center and corner of Region 1 in Figures 4-13 and 4-14 respectively show consistent trends, with slightly higher predicted levels on the $125 \mathrm{~Hz}, 160 \mathrm{~Hz}$, and $200 \mathrm{~Hz}$ bands at the center and lower predictions across most bands at the corners. These results indicate a peak in the SPL at the center of the cavity, with levels converging to the ambient-field measurement at the cavity edges.

Corresponding results for the SPL predictions in Region 2 between Panels 1 and 2 are depicted in Figures 4-15 and 4-16 for respective center and corner of the cavity. The SPL in this region seems to be largely unaffected by the size of the adjacent volume in the model, with the initial disparities in predicted levels between Regions 1 and 2, shown in Figure 4-5, retained. 


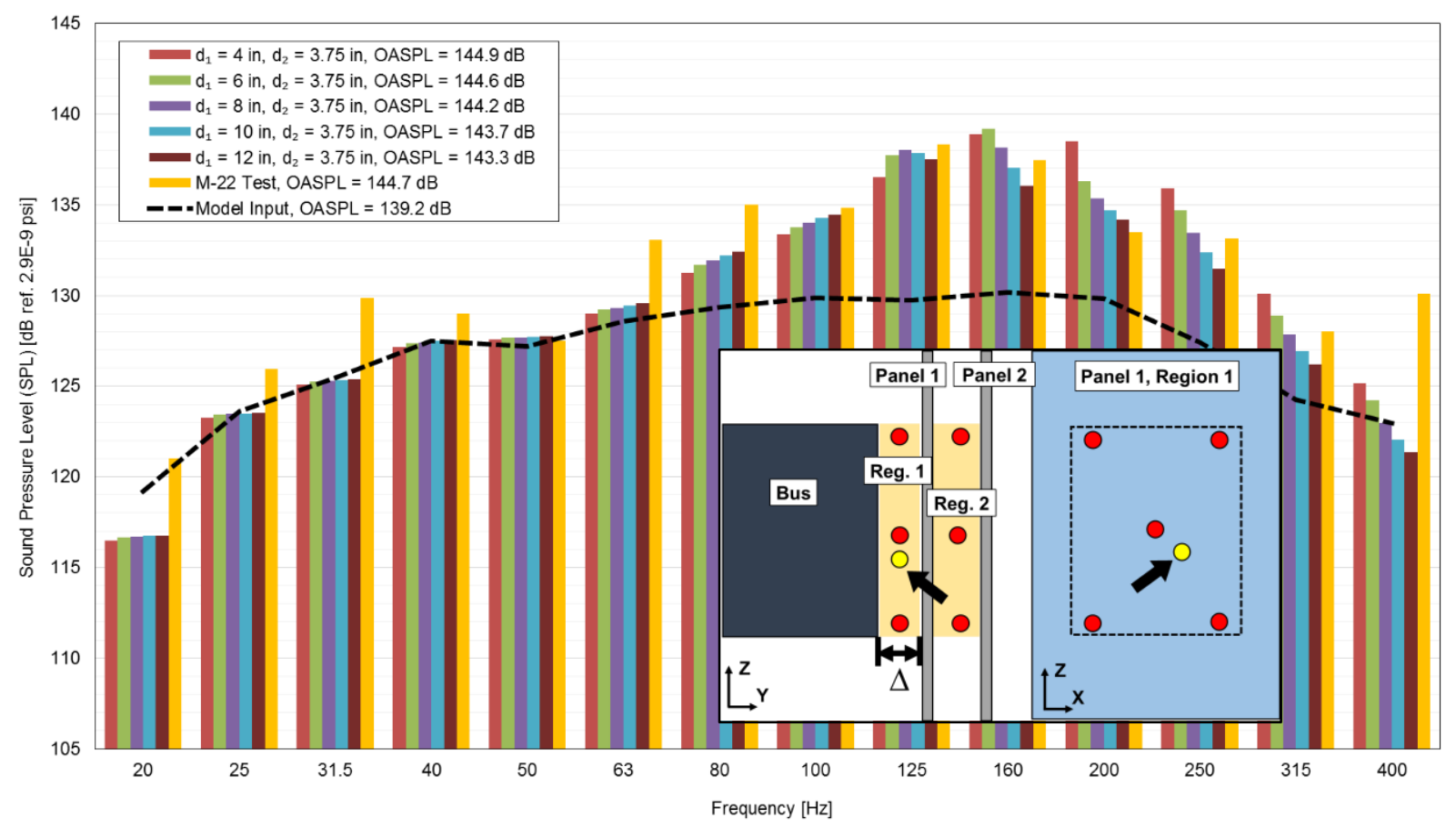

Figure 4-12. SPL Prediction at Test Microphone Location for varied d1

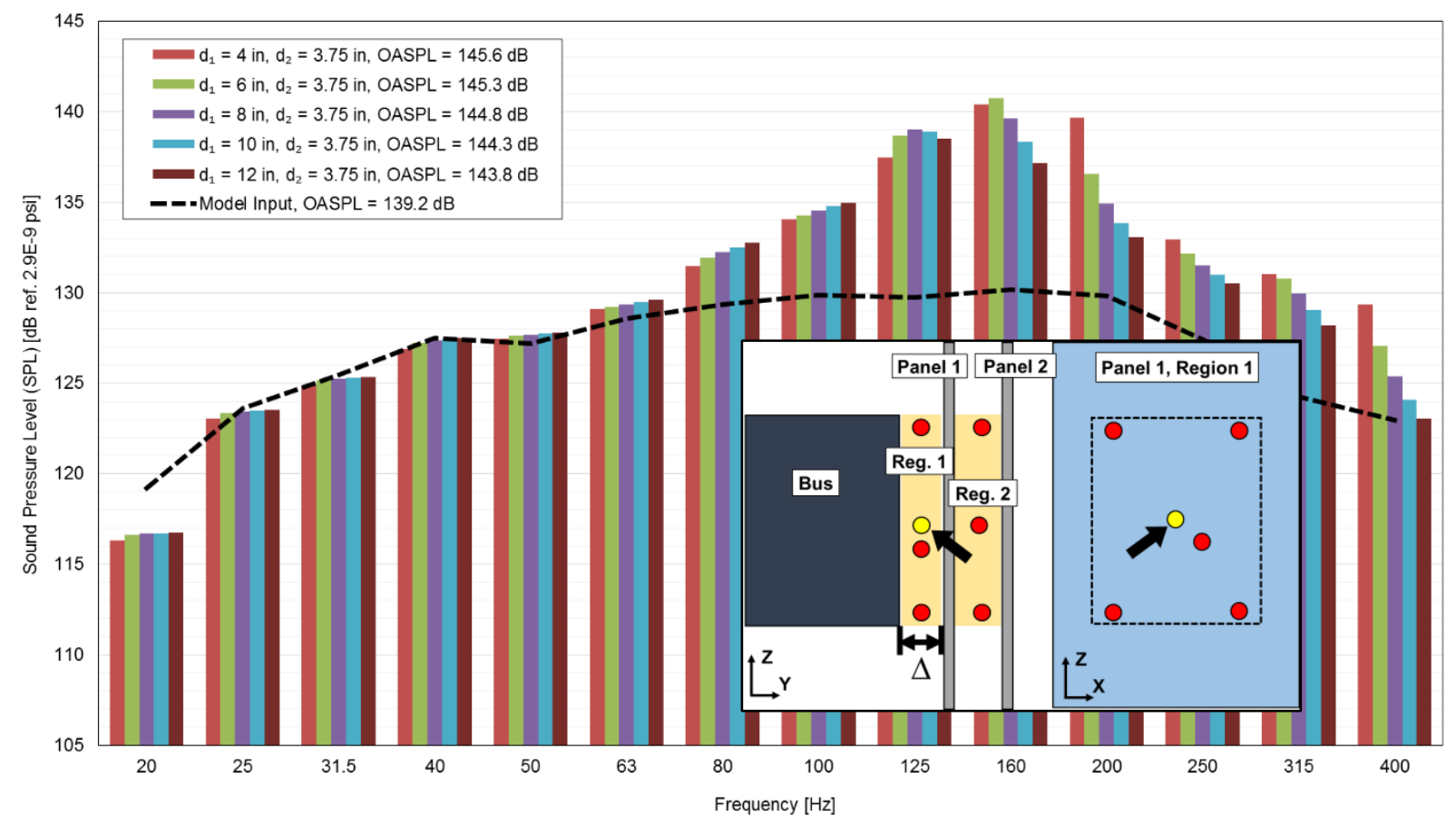

Figure 4-13. SPL Prediction in the Center of Region 1 for varied d 1 


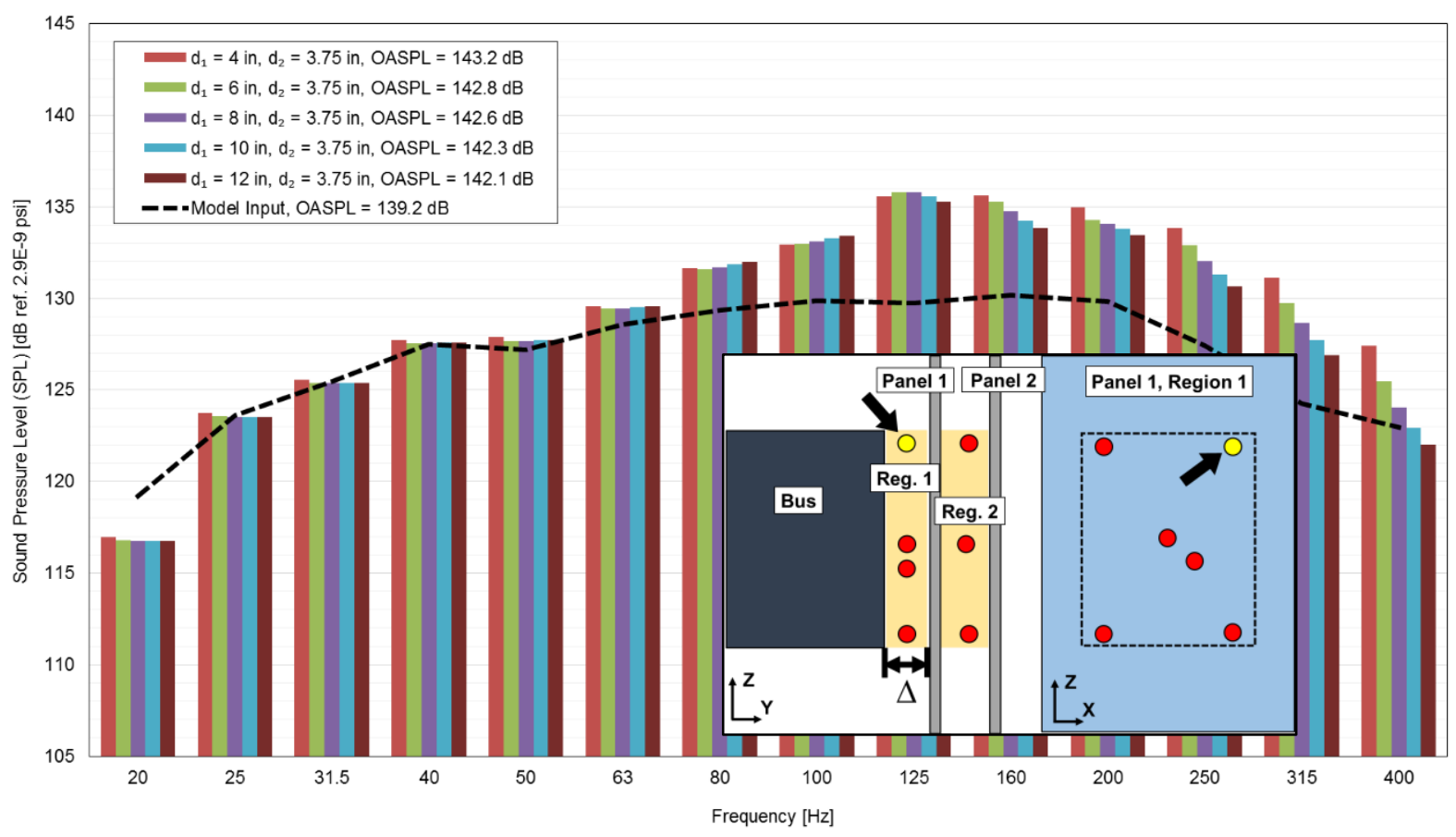

Figure 4-14. SPL Prediction at Corner of Region 1 for varied $d_{1}$

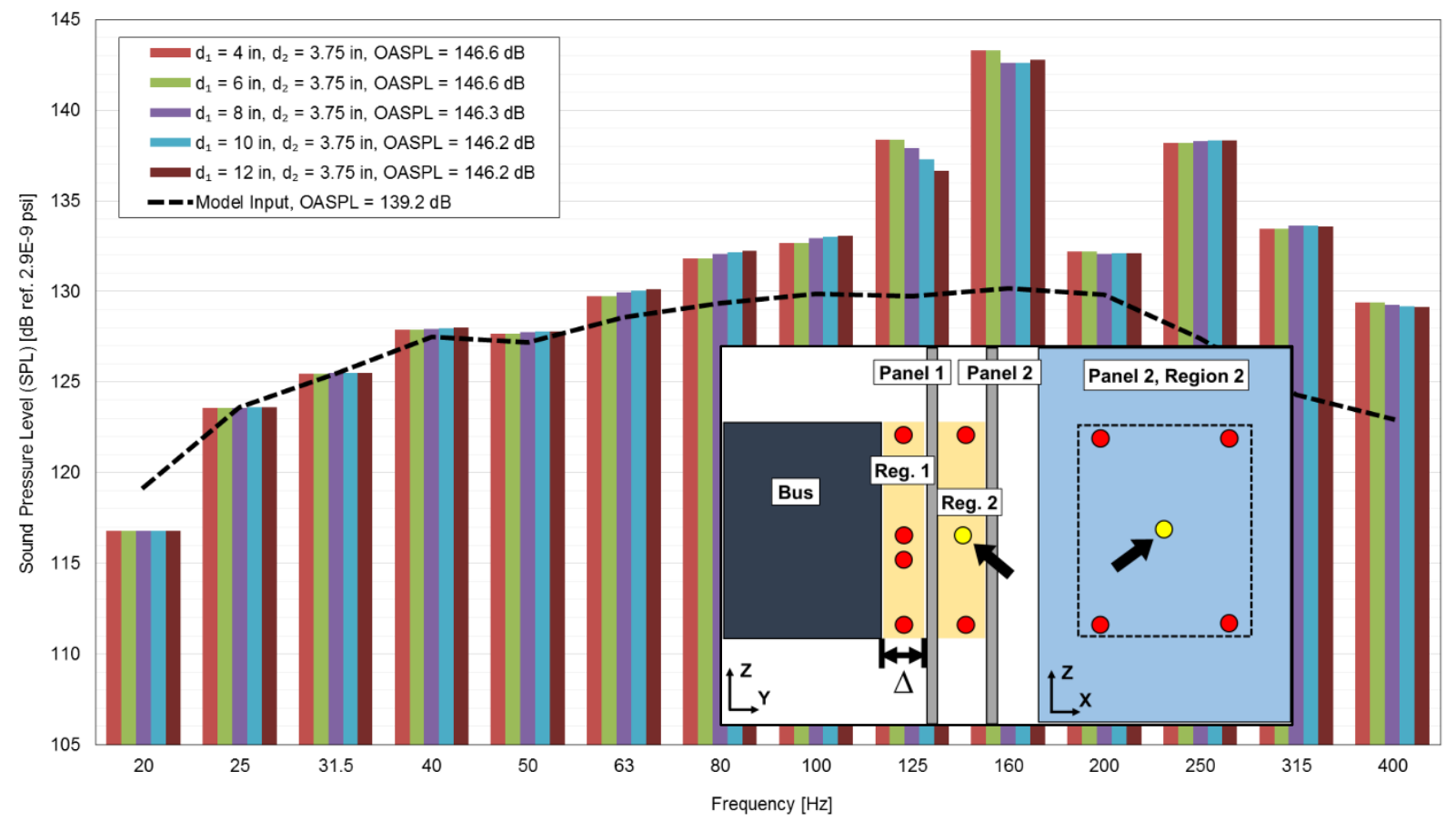

Figure 4-15. SPL Prediction in the Center of Region 2 for varied d 1 


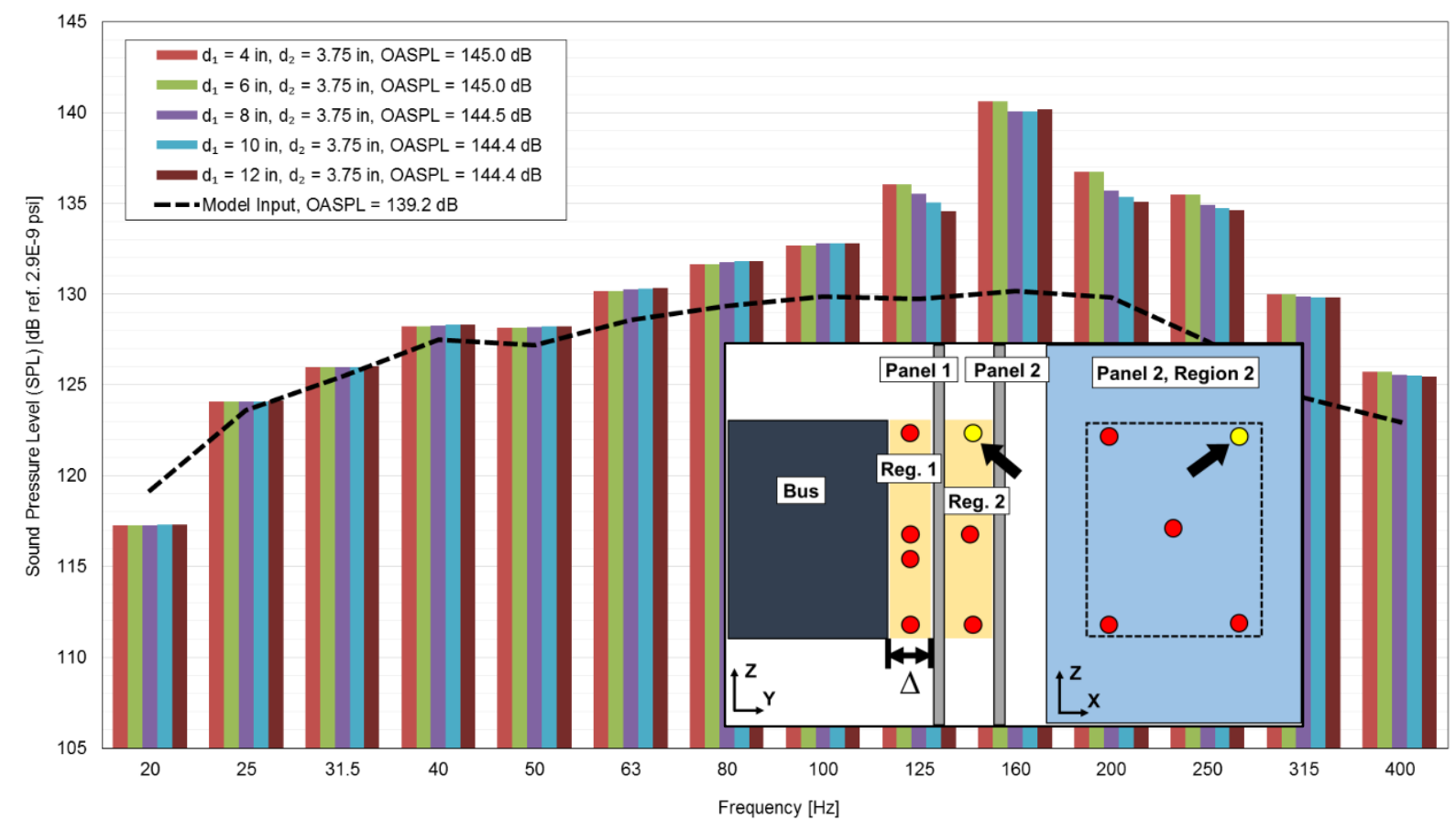

Figure 4-16. SPL Prediction at Corner of Region 2 for varied d 1

In similar fashion to the trades for the separation distance $d_{l}$ between the Bus and Panel 1, the next set of trades performed involved increasing the separation distance $d_{2}$ between Panels 1 and 2 . This parameter was also increased in 50\% (1.875") increments over the baseline value of 3.75 " to observe how the dynamic responses of the adjacent panels and the SPL distribution in the two regions would be affected. The corresponding change in the structural response prediction at the test accelerometer location is depicted in Figure 4-17. Comparing these results with those for the $d_{1}$ trades at the same location, adjusting $d_{2}$ seemed to have more influence on the response predictions below $100 \mathrm{~Hz}$, particularly at the first mode around $50 \mathrm{~Hz}$. Some response variation is seen at $150 \mathrm{~Hz}$, but otherwise the response prediction at the higher frequencies remains unchanged. Similar variations are seen for the response predictions at the center and corners of Panel 1 in Figures 4-18 and 4-19, respectively. The $d_{2}$ trade had more widespread influence on the Panel 2 response predictions as shown in Figures 4-20 and 4-21. At the center of the panel, there are variations at the $50 \mathrm{~Hz}$ and $80 \mathrm{~Hz}$ modes, and also along the $100 \mathrm{~Hz}$ to $175 \mathrm{~Hz}$ bandwidth. At the 
corners of the panel, there is a consistent variation at the $50 \mathrm{~Hz}$ mode, and also for the peak response at $130 \mathrm{~Hz}$.

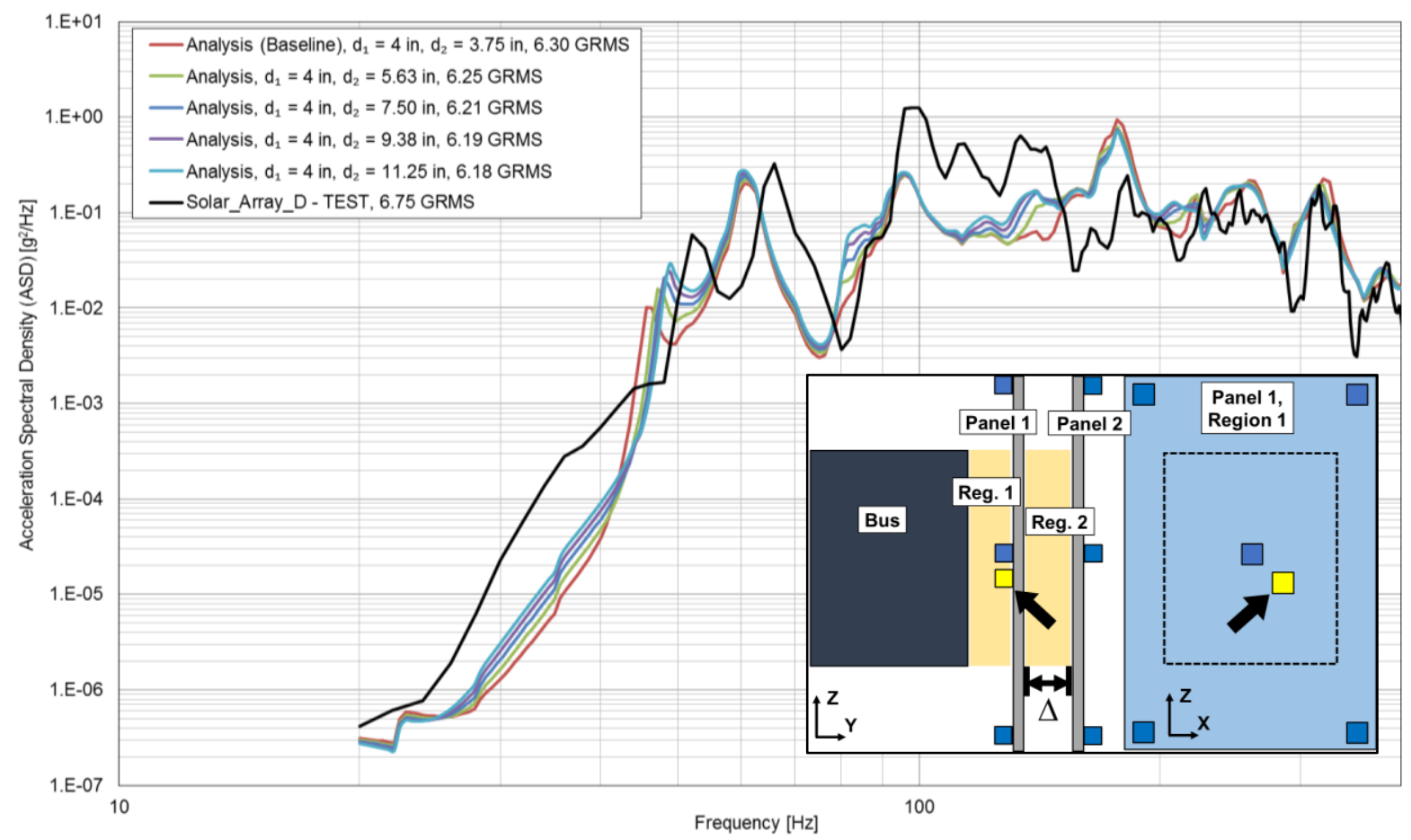

Figure 4-17. ASD Prediction at Test Accel Location for Varied d2 


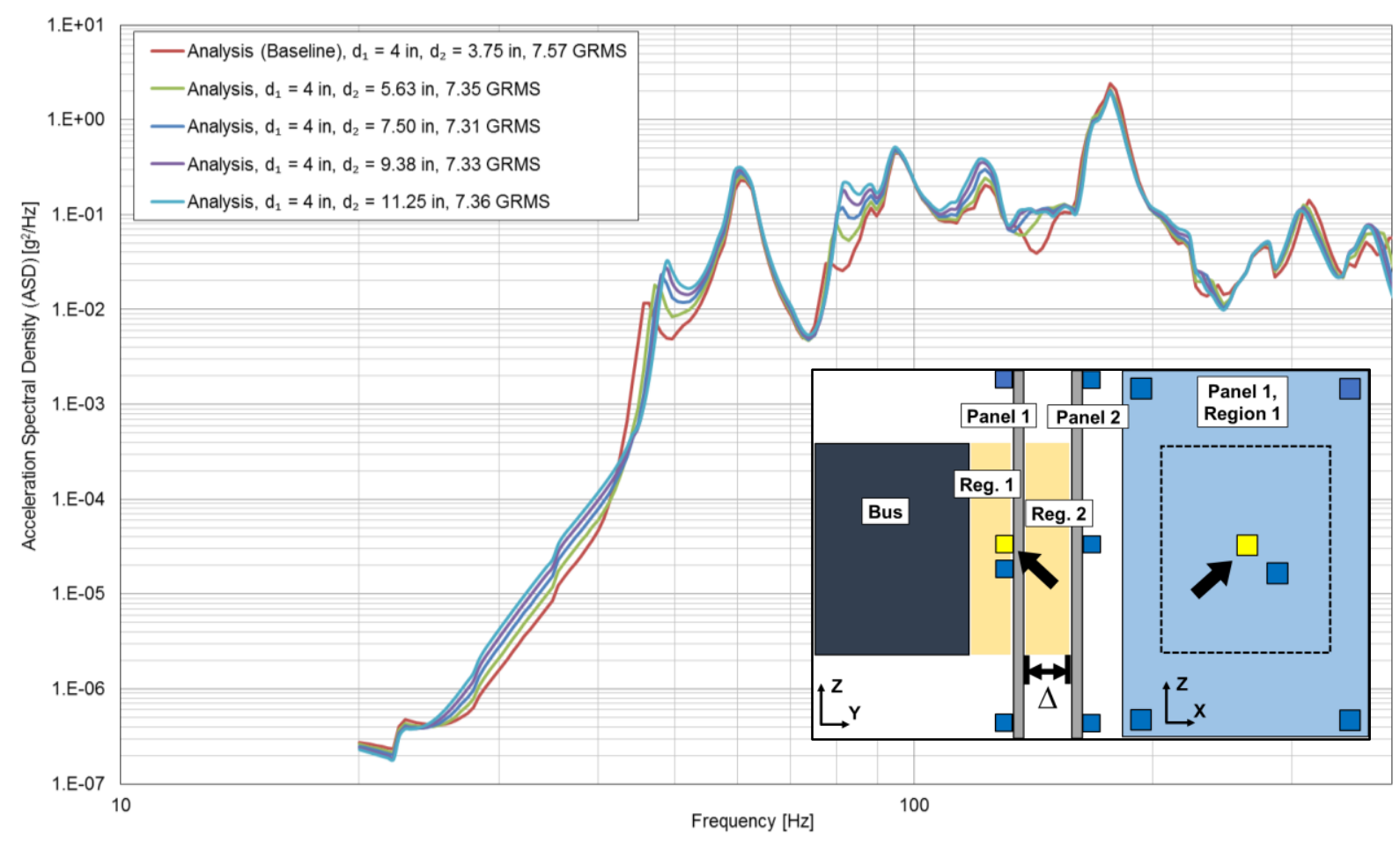

Figure 4-18. ASD Prediction at Panel 1 Center for Varied $d_{2}$

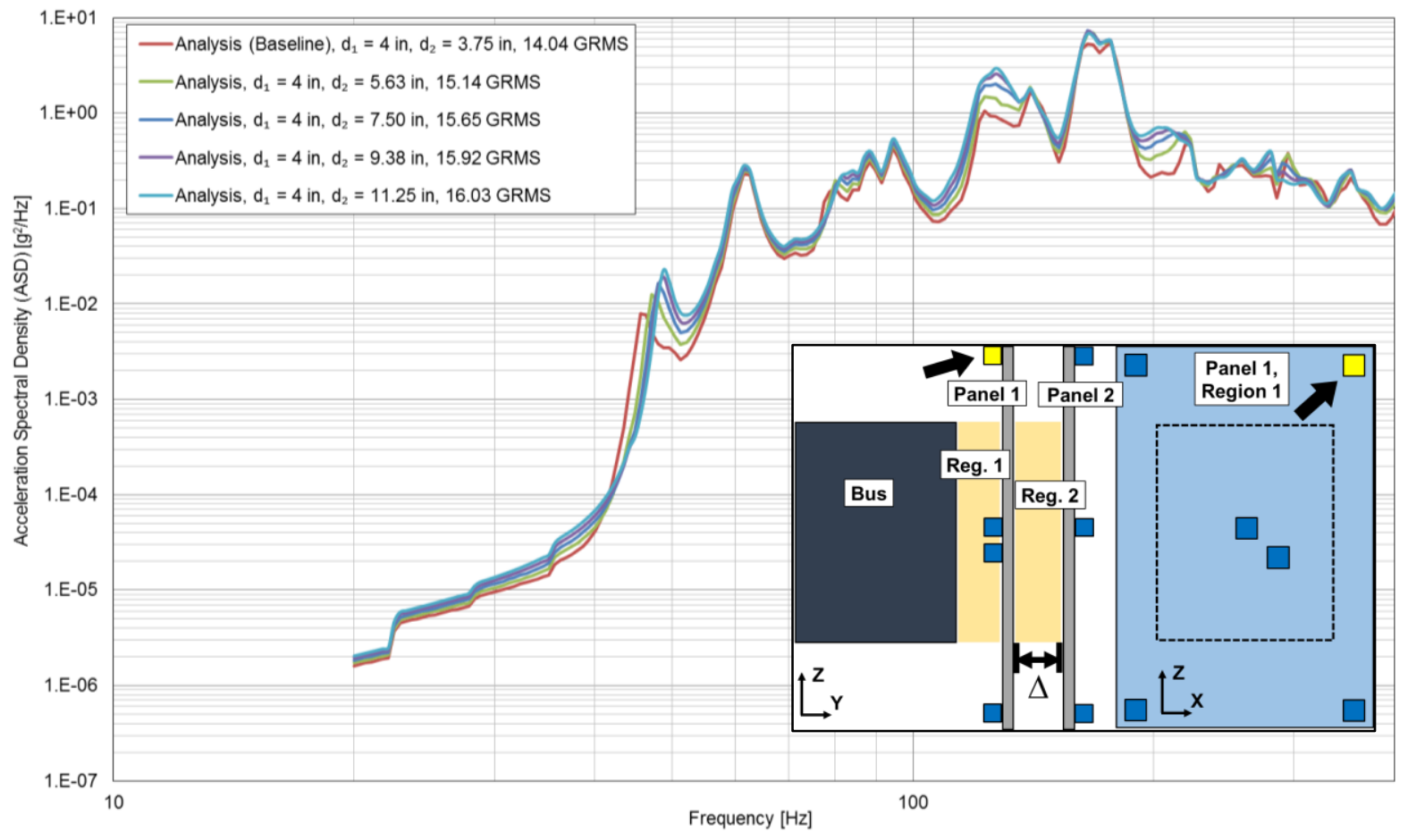

Figure 4-19. ASD Prediction at Panel 1 Top Corner for Varied $\mathbf{d}_{2}$ 


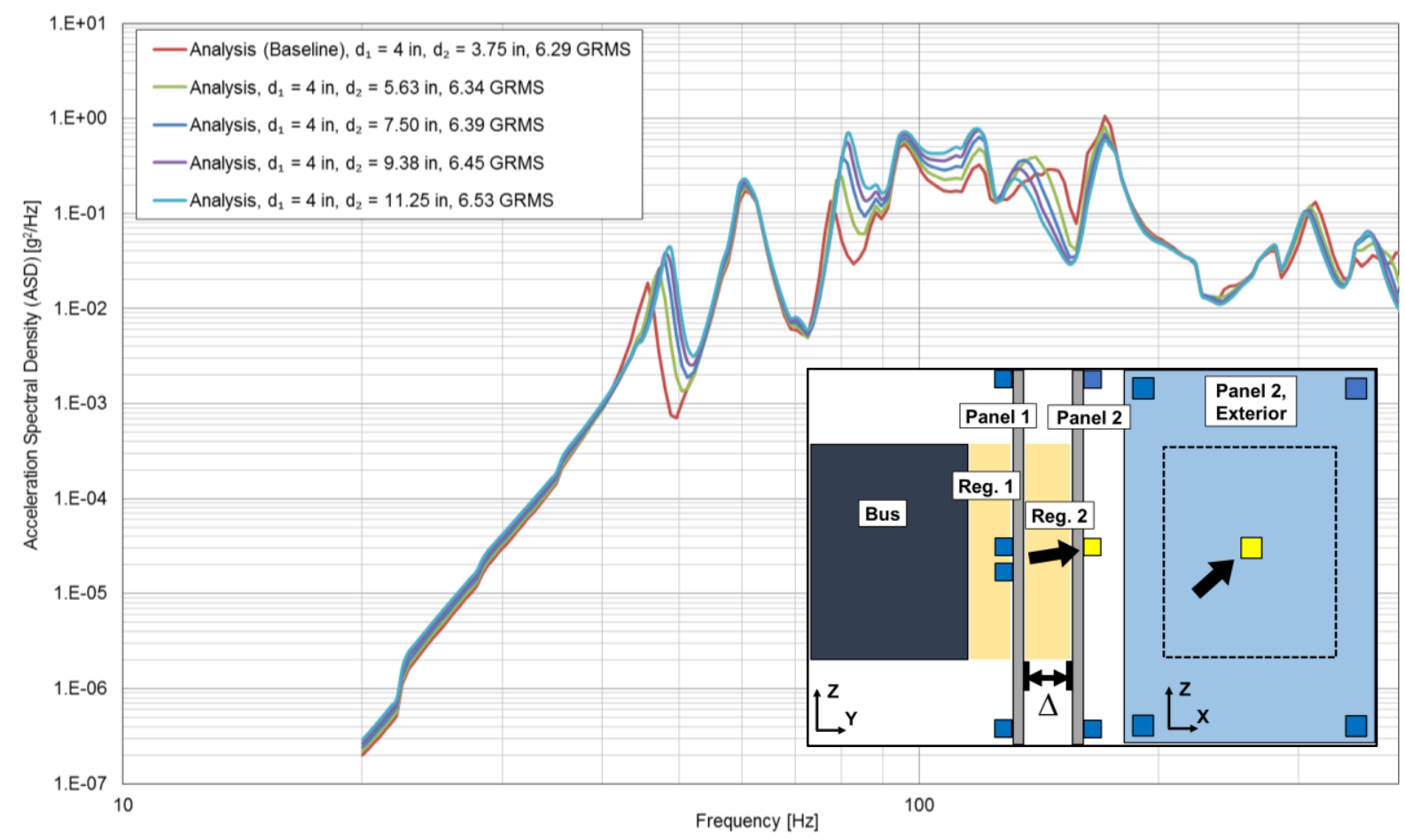

Figure 4-20. ASD Prediction at Panel 2 Center for Varied d2

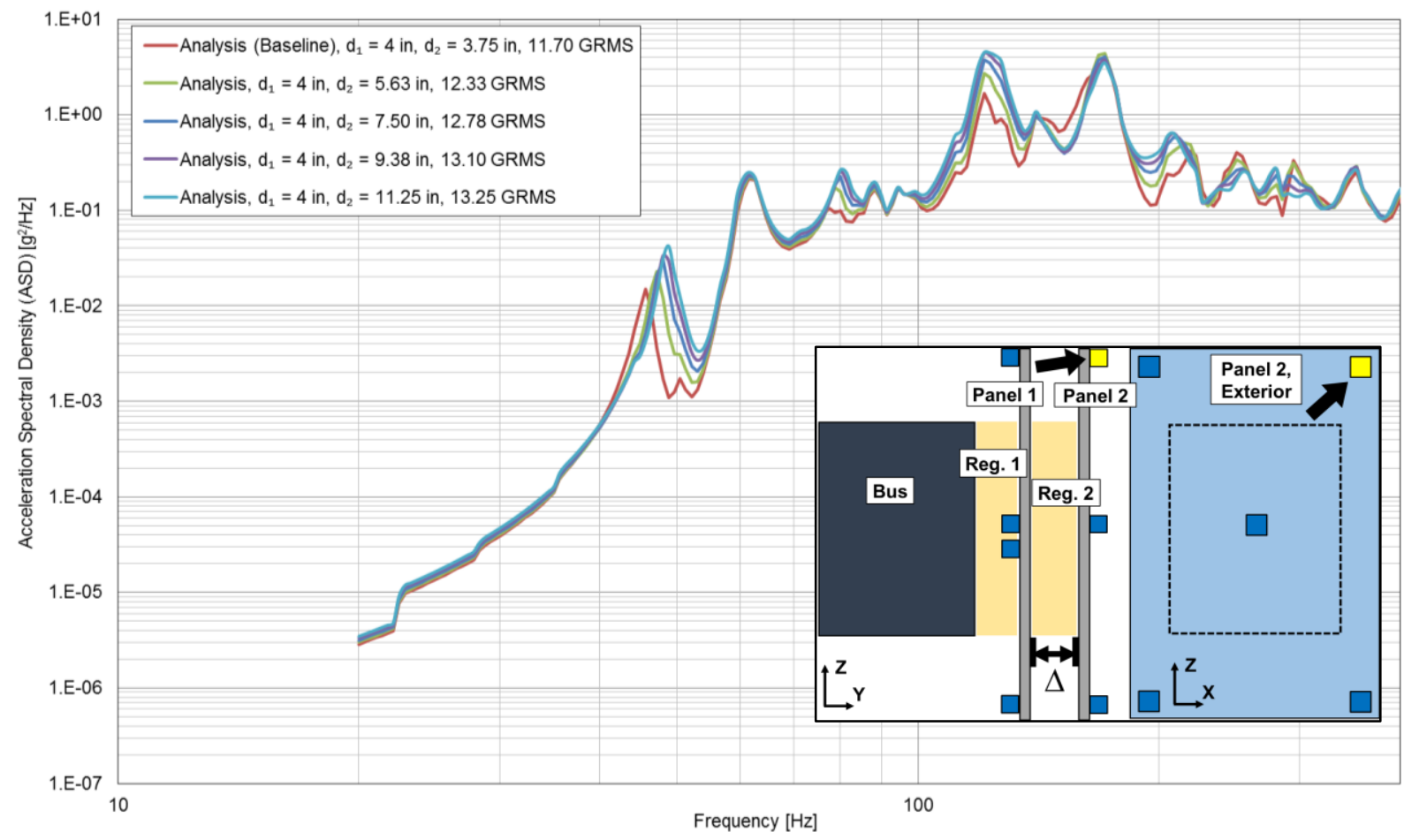

Figure 4-21. ASD Prediction at Panel 2 Top Corner for Varied d2 
The SPL predictions at locations in Region 1 for the $d_{2}$ trades shown in Figures 4-22, 4-23, and 4-24 are consistent with the predictions in Region 2 for the $d_{1}$ trades in that the local acoustic field between panels with unchanged separation distance seems to be largely unaffected, with the exception of the consistent decrease seen at all three locations on the $125 \mathrm{~Hz}$ band. In contrast, the SPL predictions for the respective center and corner points of Region 2 shown in Figures 4-25 and 4-26 are affected for the $80 \mathrm{~Hz}$ frequency band and above. The most significant variation is seen at the center of Region 2 where the predicted SPL drops from a peak value of $143 \mathrm{~dB}$ at the baseline distance $d_{2}=3.75 "$ to $134 \mathrm{~dB}$ for $d_{2}=11.25$ ".

In general, it was observed that the SPL predictions for different locations in Regions 1 and 2 were most influenced by changes in the local volume resulting from increased separation between the adjacent panels. Otherwise, for fixed local volume in a region $R_{i}$ corresponding to a consistent separation distance $d_{i}$, the SPL predictions were largely unaffected.

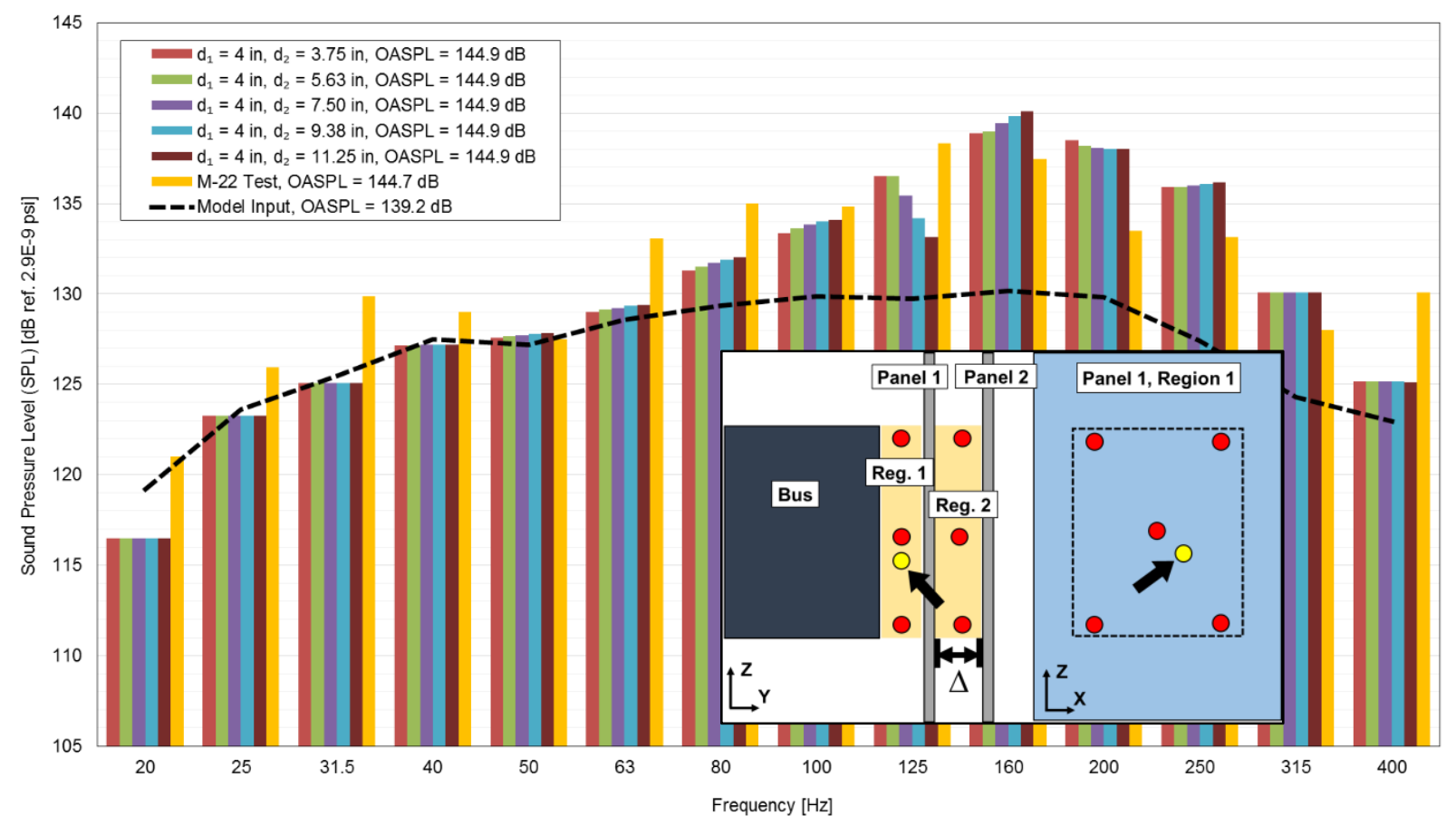

Figure 4-22. SPL Prediction at M-22 Microphone Location for Varied d2 


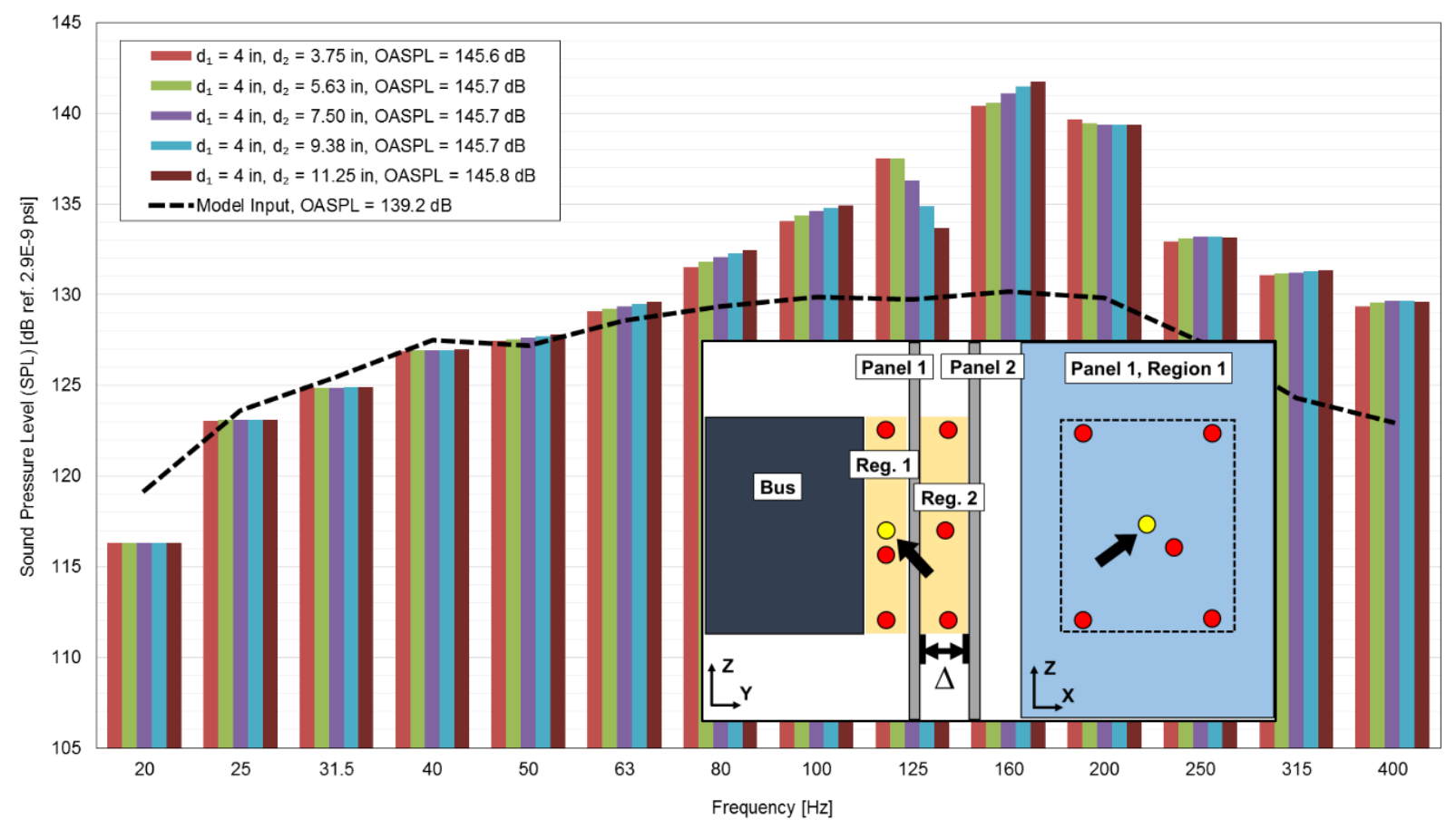

Figure 4-23. SPL Prediction in the Center of Region 1 for varied d2

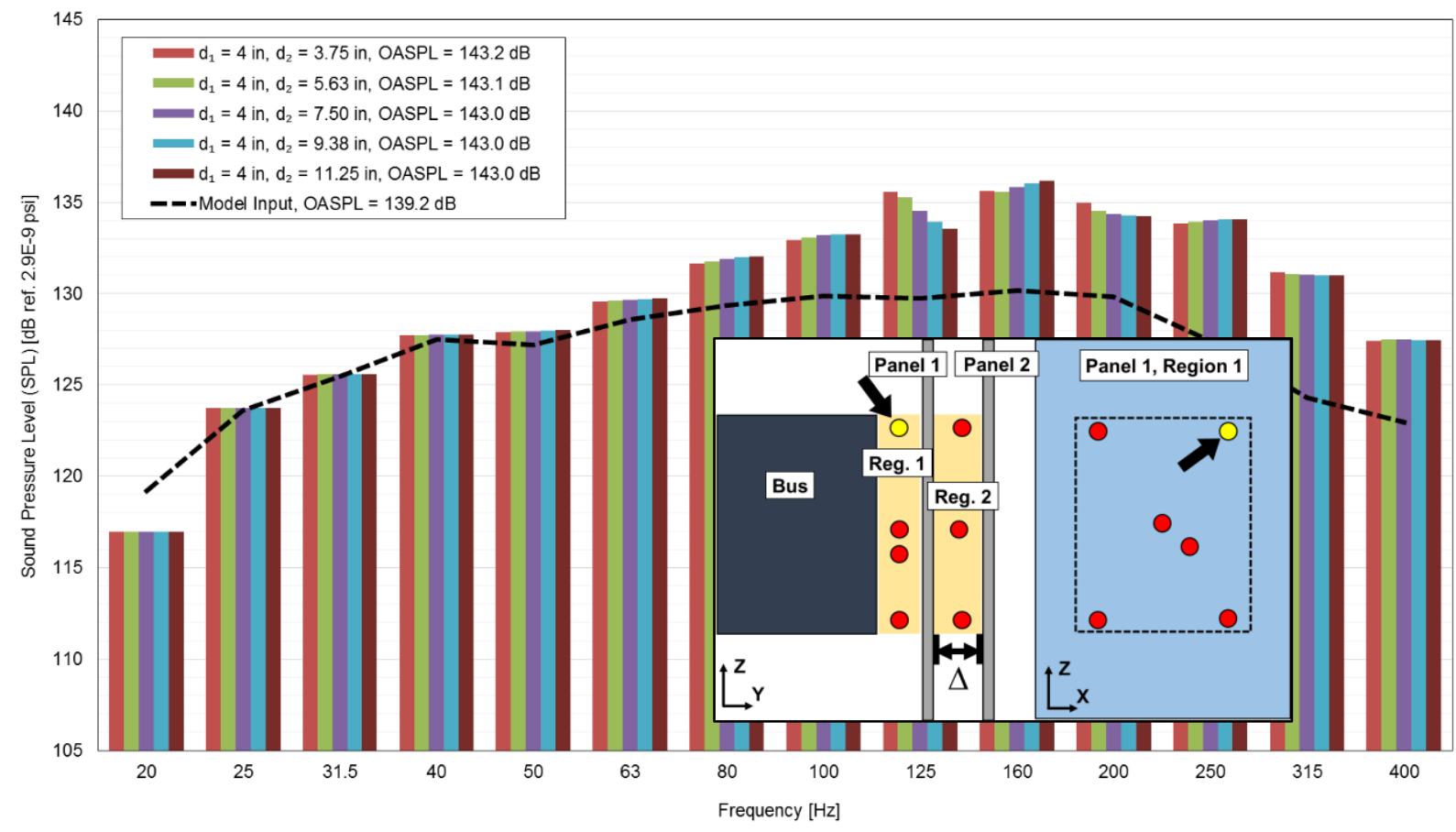

Figure 4-24. SPL Prediction at Corner of Region 1 for varied $d_{2}$ 


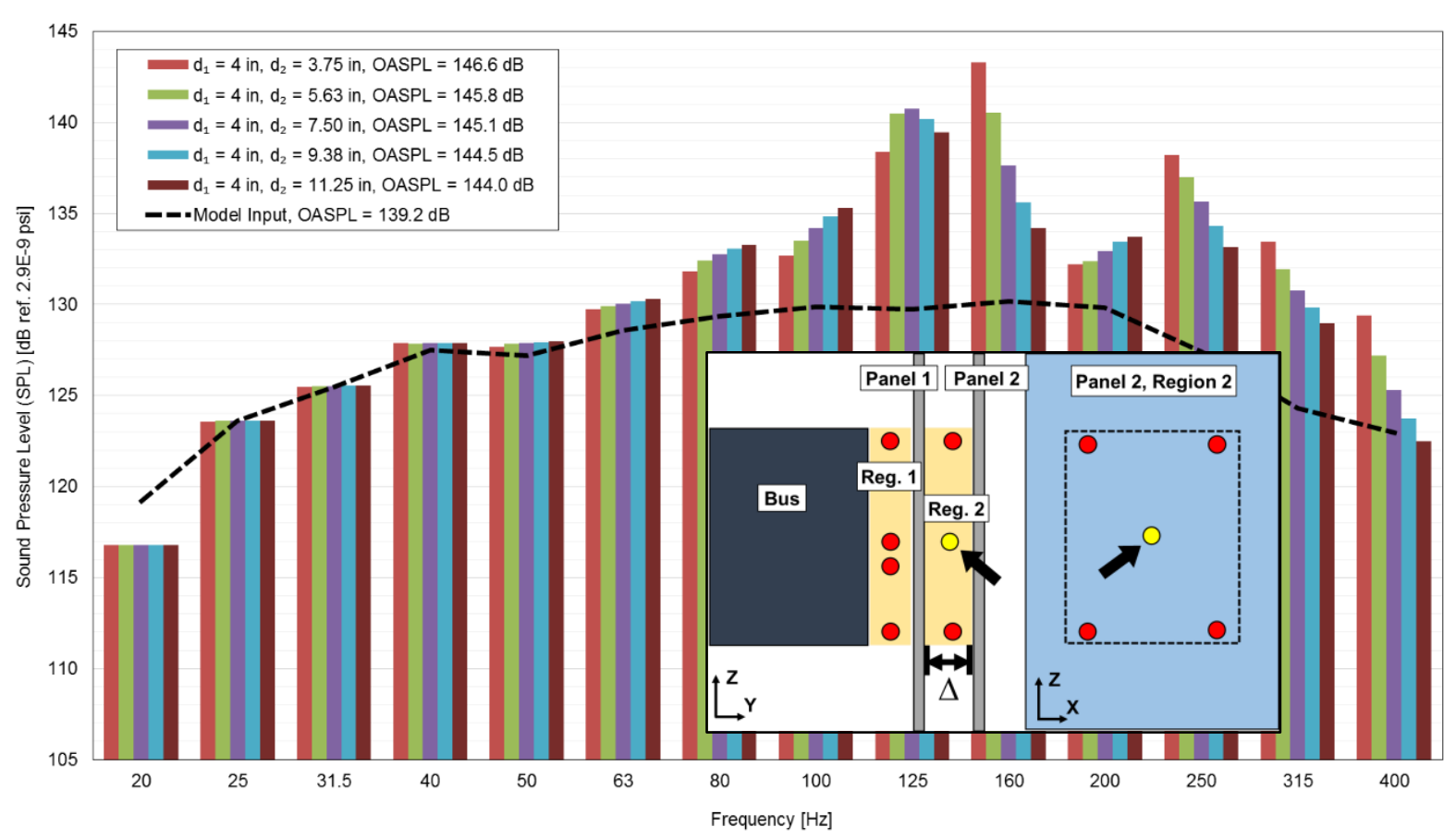

Figure 4-25. SPL Prediction at the Center of Region 2 for varied d2

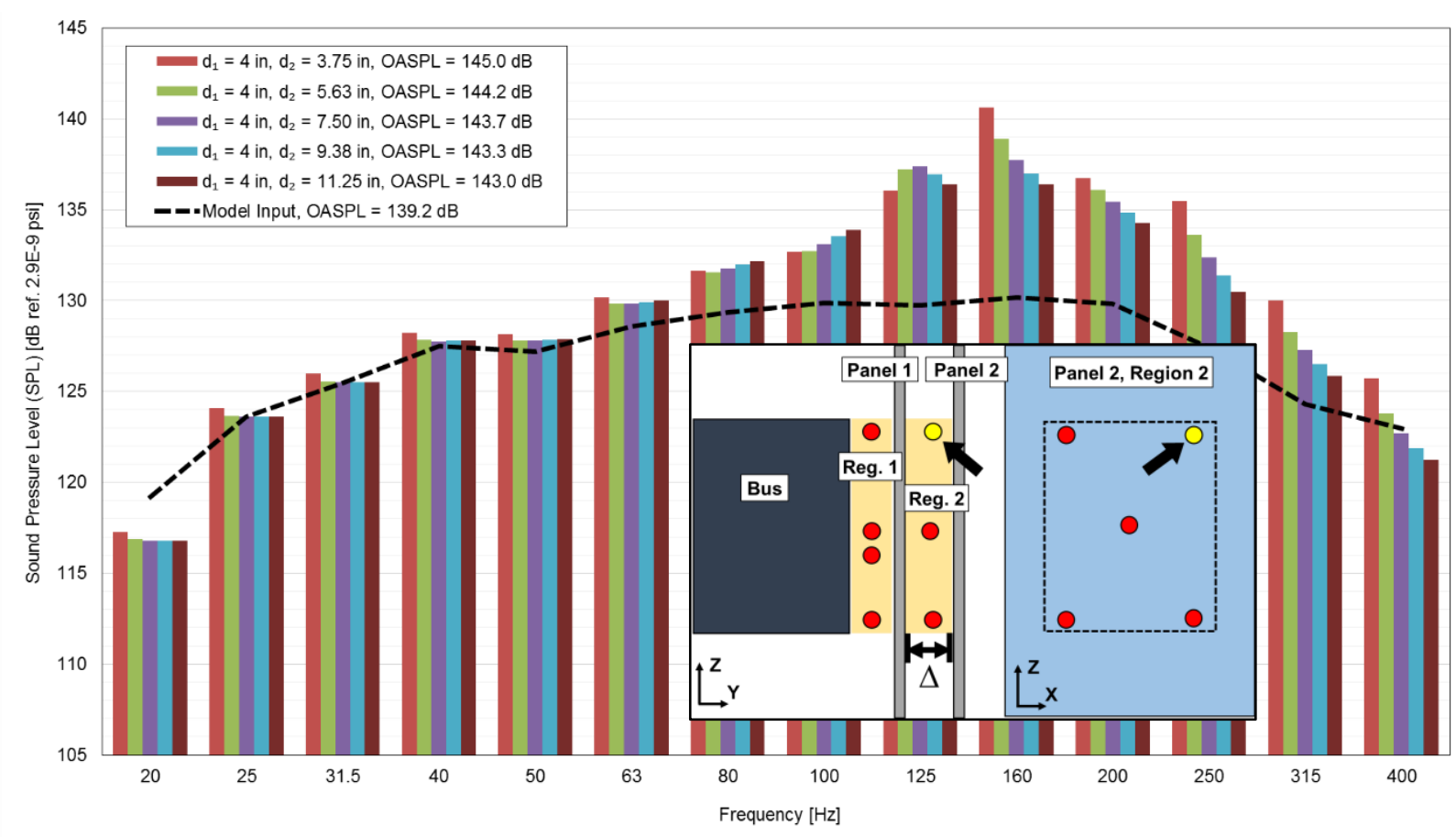

Figure 4-26. SPL Prediction at Corner of Region 2 for varied $d_{2}$

In compliment to Figure 4-6, Figure 4-27 combines data from Figures 4-7 and 4-12 to depict how the energy distribution in the system is sensitive to the size of the cavity volume between panels. 
As $d_{l}$ is increased, Figure 4-27 illustrates a consistent shift in the structural modes of Panel 1 and the acoustic modes of Region 1 across the $20-400 \mathrm{~Hz}$ bandwidth, indicating that the analytically coupling present in the baseline model is maintained for this trade. It is also evident that as the cavity volume of Region 1 is increased, the acoustic energy in that cavity decreases - this is an expected result because increasing $d_{l}$ provides proportionally larger openings for energy dissipation and it confirms that the model is sensitive to the spacing between adjacent panels.

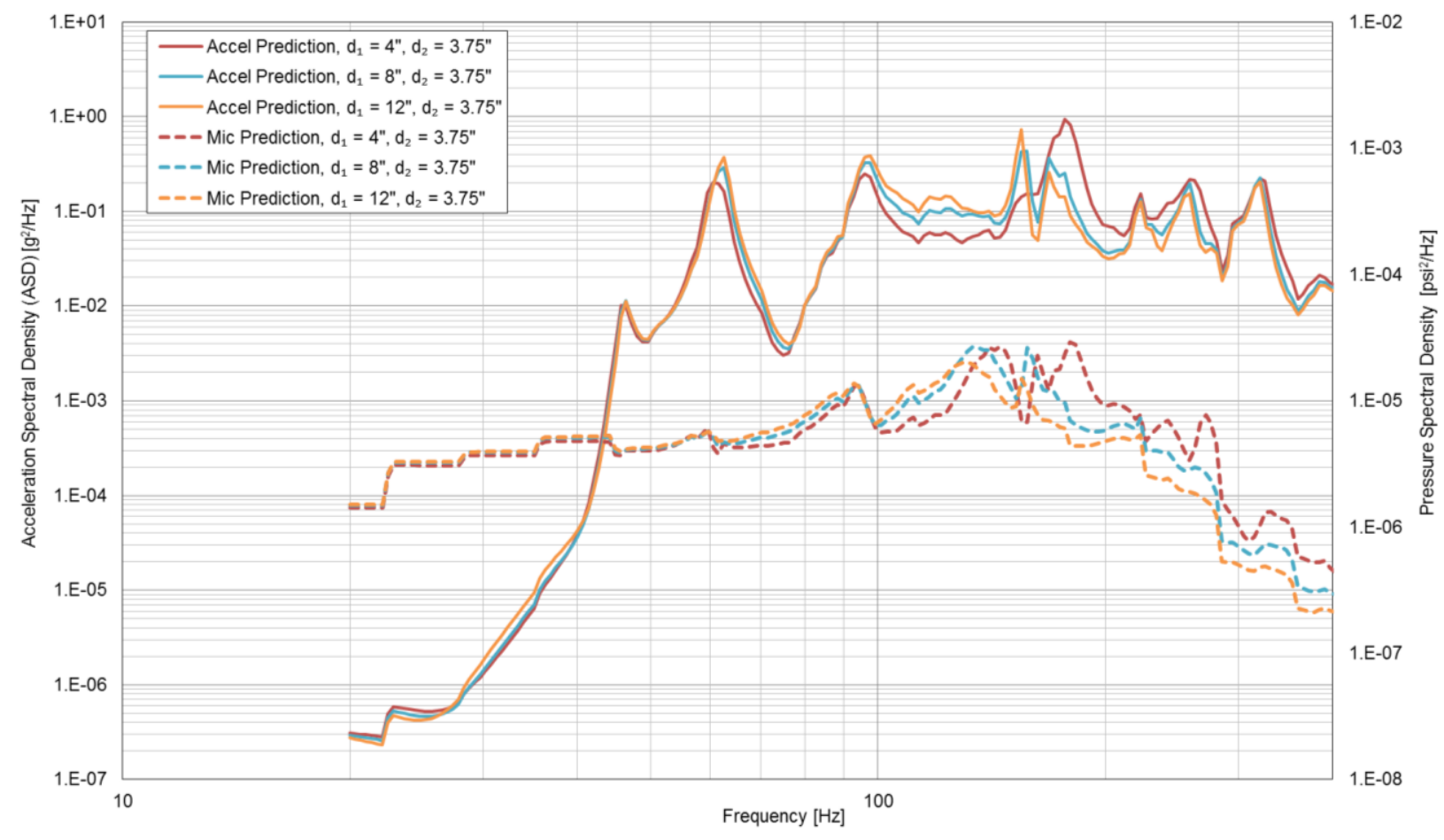

Figure 4-27. Analytical Coupling between Panel 1 Structural Modes and Region 1 Cavity Modes for varied $d_{1}$ 


\subsection{Damping Loss Factor Trades}

The response prediction at the test accelerometer location was obtained for different structural damping assumptions. Before being imported to the acoustic model, the structural modes take into account the mass loading from the air incident on the solar array panels. By standard practice, an additional damping loss factor (DLF), equivalent to twice the damping ratio, was specified for the FE structures across all frequency bands in the coupled acoustic solution in VA One (see Appendix A for more information) and the corresponding impact on the structural response predictions was observed. This change in the assumed damping only affects the narrow-band response peaks with no alteration in frequency. The DLF was traded for values ranging from $1 \%$ to $4 \%$ and the results are presented in Figure 4-28. Based on this, it can be concluded that the chosen baseline DLF of $4 \%$ is acceptable; this value was selected for the rest of the trades since it is a value often assumed in standard practice.

The structural damping can be adjusted to account for response peaks at specific frequencies, but this tailored damping spectrum would then only be valid at that specific location for the given acoustic excitation. In standard practice, the DLF would be assumed based on analytical comparison with test data at a number of locations; however in this case the assumption is limited to a single data point. 


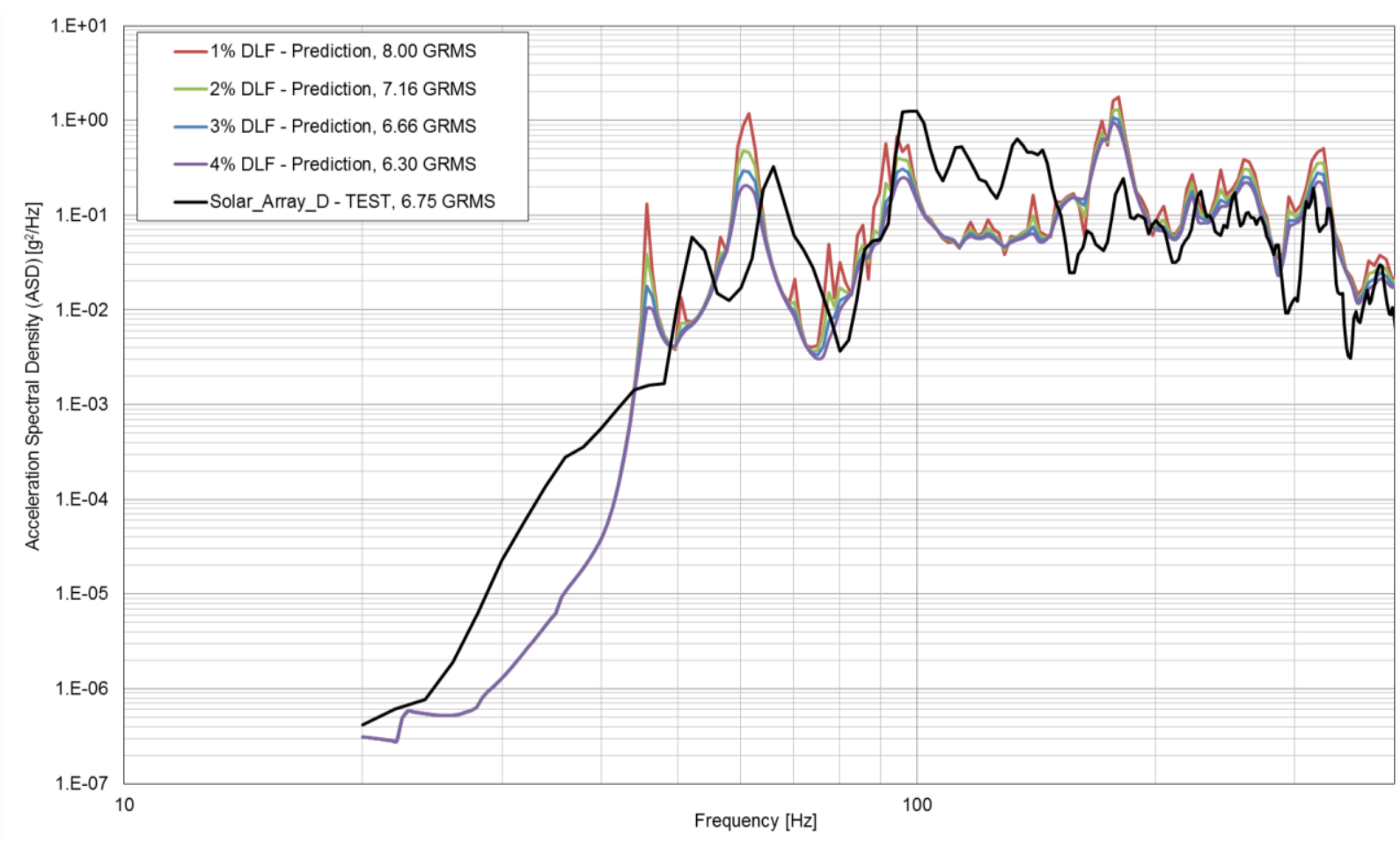

Figure 4-28. ASD Prediction at Solar_Array_D Accelerometer Location for Varied Damping Loss Factor Applied Across All Frequencies

\subsection{BE Mesh Size Trades}

The final set of trades in this work took the form of a mesh convergence study to investigate the sensitivity of the model solution to the BE mesh size - this involved performing the intermediate BEM solution for the fluid domain and final coupled acoustic solution for different BE mesh sizes. The corresponding effects on the BEM solution CPU time and relative shifts in structural response and SPL predictions at the test recovery locations were observed. The CPU time for the BEM solution is governed by the number of "wetted" nodes considered in the BE fluid domain and follows an exponential relationship, so it is well known that increased mesh fidelity can quickly lead to higher computational expense, but it was not known how this mesh would influence the accuracy of analytical predictions. The baseline model was solved for BE mesh sizes ranging from 2" to $10 "$ and the resulting analytical predictions are shown in Figures 4-29 and 4-30. The 
analytical results for both the structural response at the accelerometer location and the SPL at the microphone location are consistent in that negligible variation is seen for BE mesh sizes ranging from 2" to 8". For these mesh sizes, the analytical results are largely independent of the nodal density; however, for a BE mesh size of 10", the results show a clear divergence from the baseline in both cases. In both plots, the maximum recommended analysis frequency for each mesh size is given in the legend; these values are based on the allocated 6 elements per incident sound wavelength. For instance, a 2" BE mesh size is valid for frequencies over $1100 \mathrm{~Hz}$, but this benefit is lost in this analysis bandwidth which is limited to $400 \mathrm{~Hz}$.

The size of the BE mesh in the model is important for a few different reasons. First, it dictates the applicable solution bandwidth in the BEM, with a smaller mesh size corresponding to a wider analysis bandwidth and vice versa. Secondly, it influences the ability of the BE surfaces to capture the projected FE mode shapes. At higher frequencies, the structural mode shapes exhibit many inflection points and sufficient mesh density is needed for accurate modal interpolation in the final coupled acoustic solution. Lastly, the increased nodal density can have a profound impact on CPU time for the intermediate solution, as shown in Figure 4-31. The CPU time for a 2" BE mesh was over 11.5 hours with no discernable impact on the solution itself, while the same results could be obtained with a 6" BE mesh in less than 0.5 hours. These results validate the baseline BE mesh size of 6" used throughout this work. 


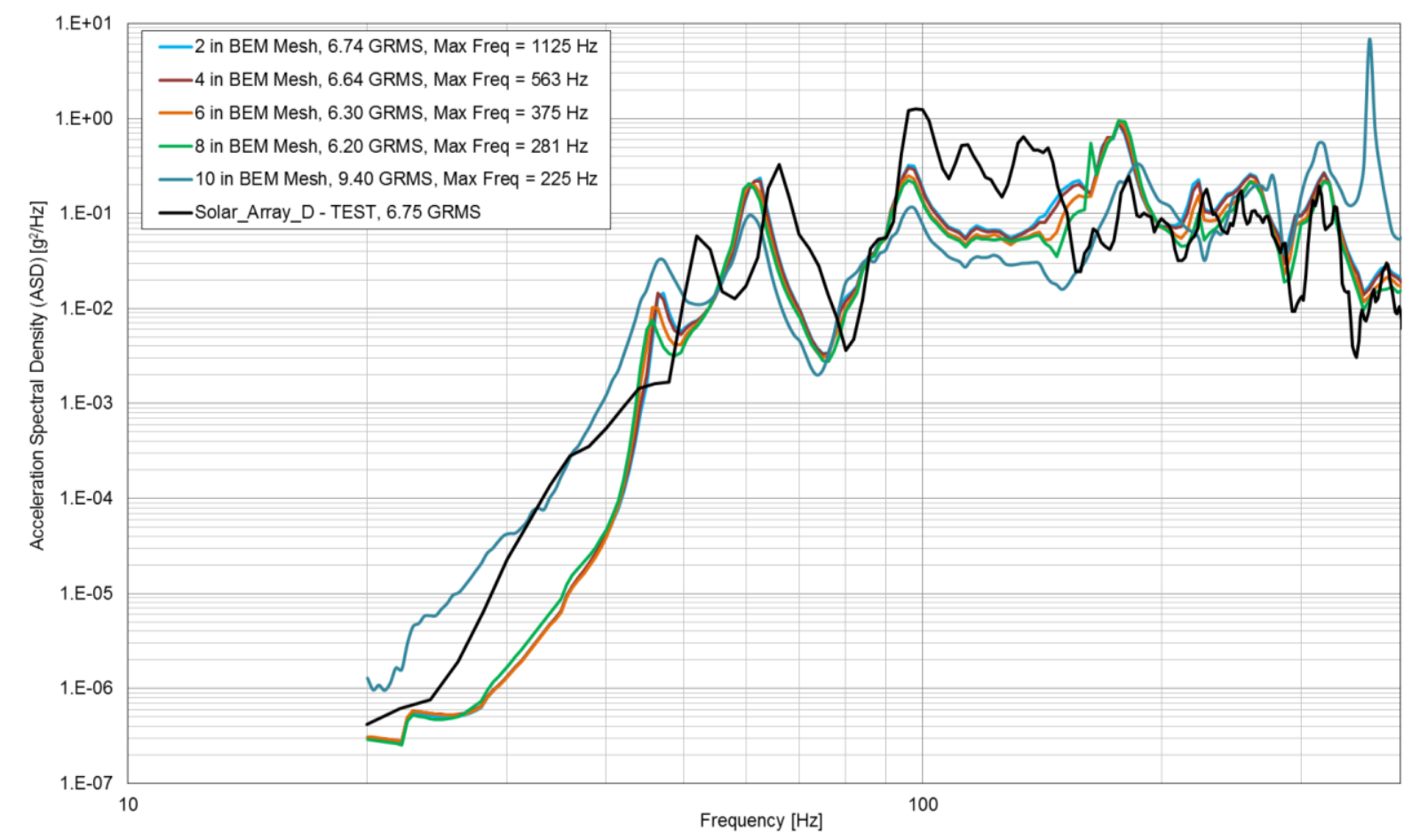

Figure 4-29. ASD Prediction at Solar_Array_D Accelerometer Location for Different BEM Mesh Sizes

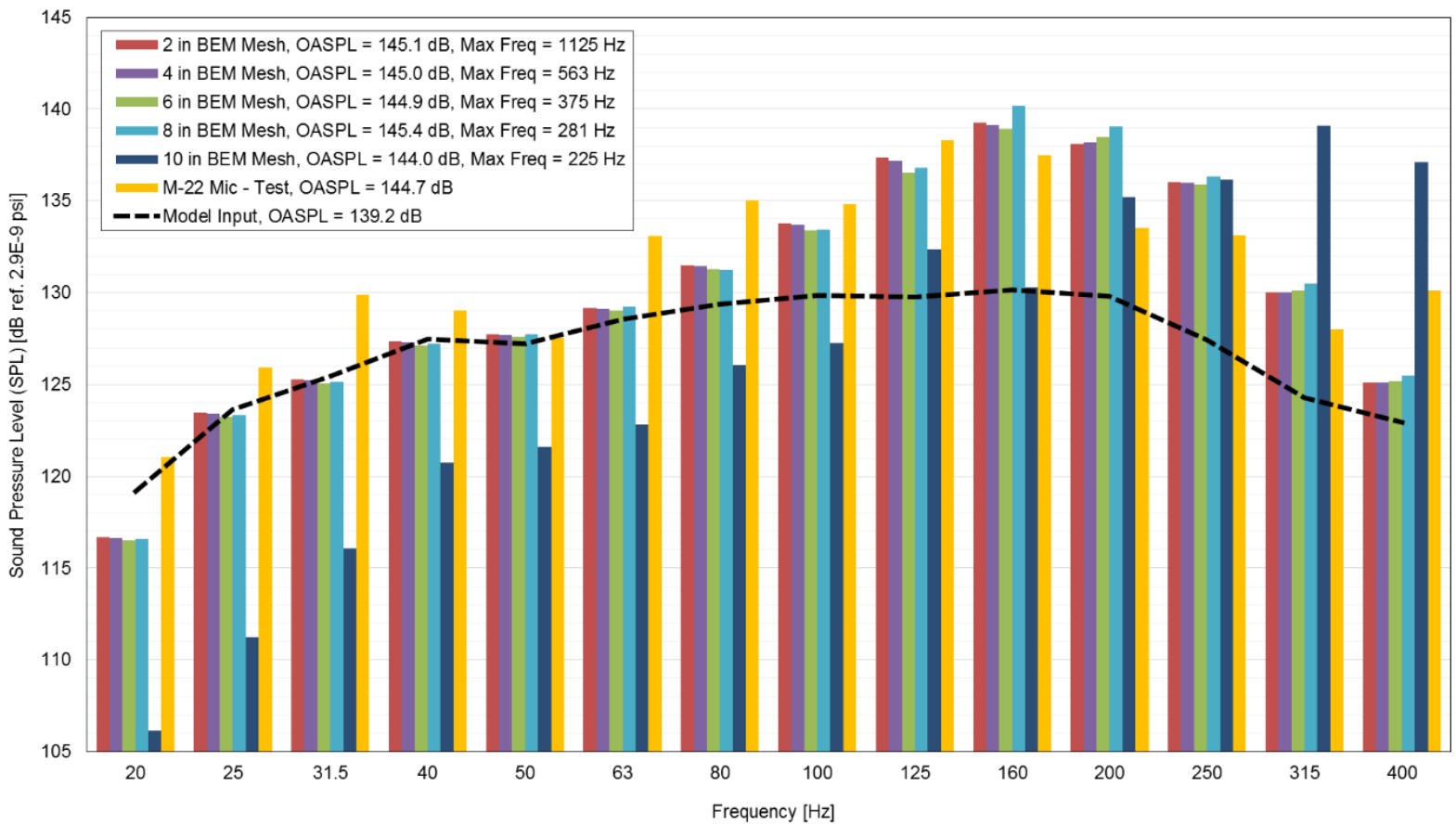

Figure 4-30. SPL Predictions at M-22 Microphone Location for Different BEM Mesh Sizes 


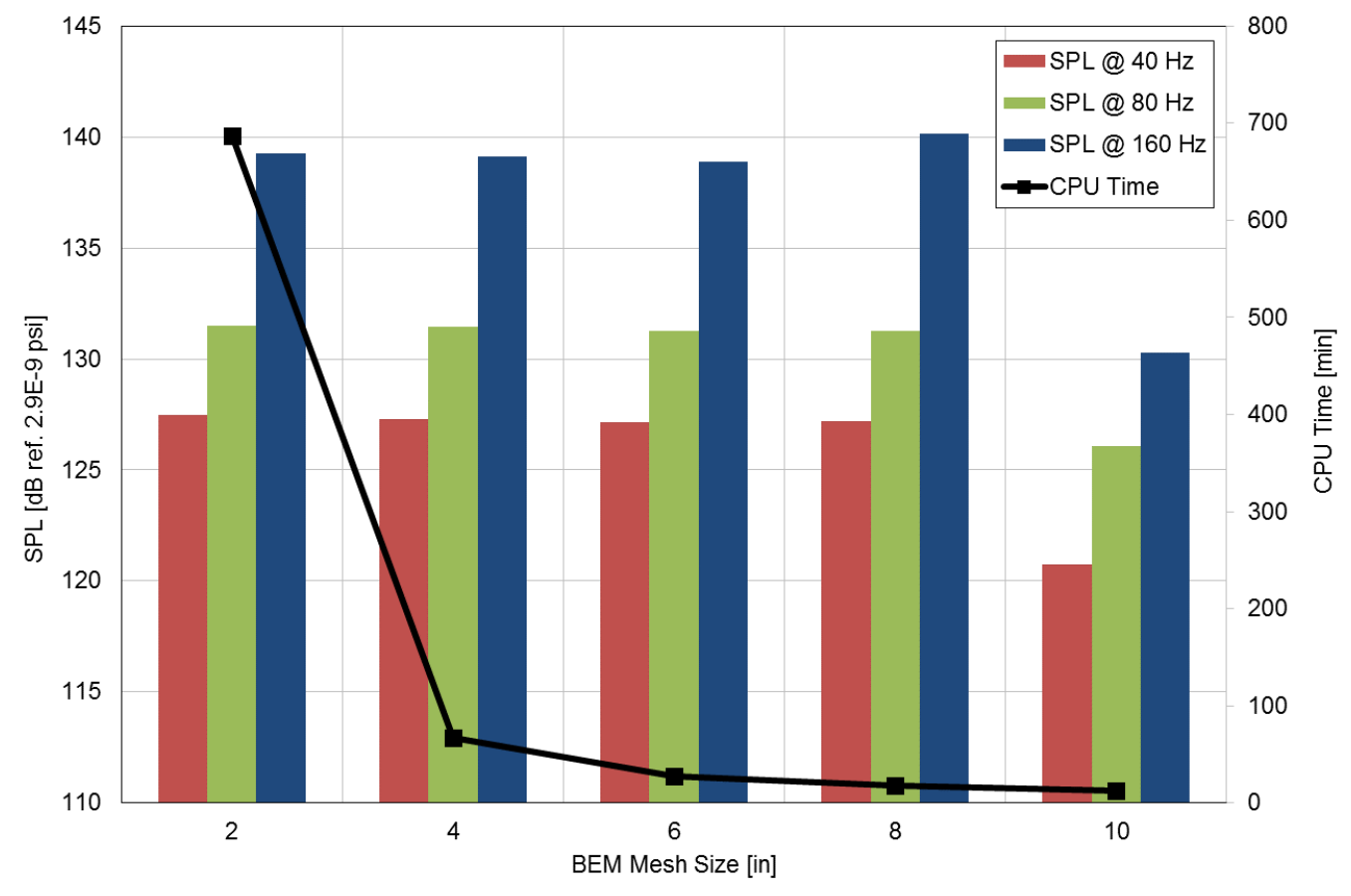

Figure 4-31. SPL Prediction and BEM Solution CPU Time for Different BEM Mesh Sizes
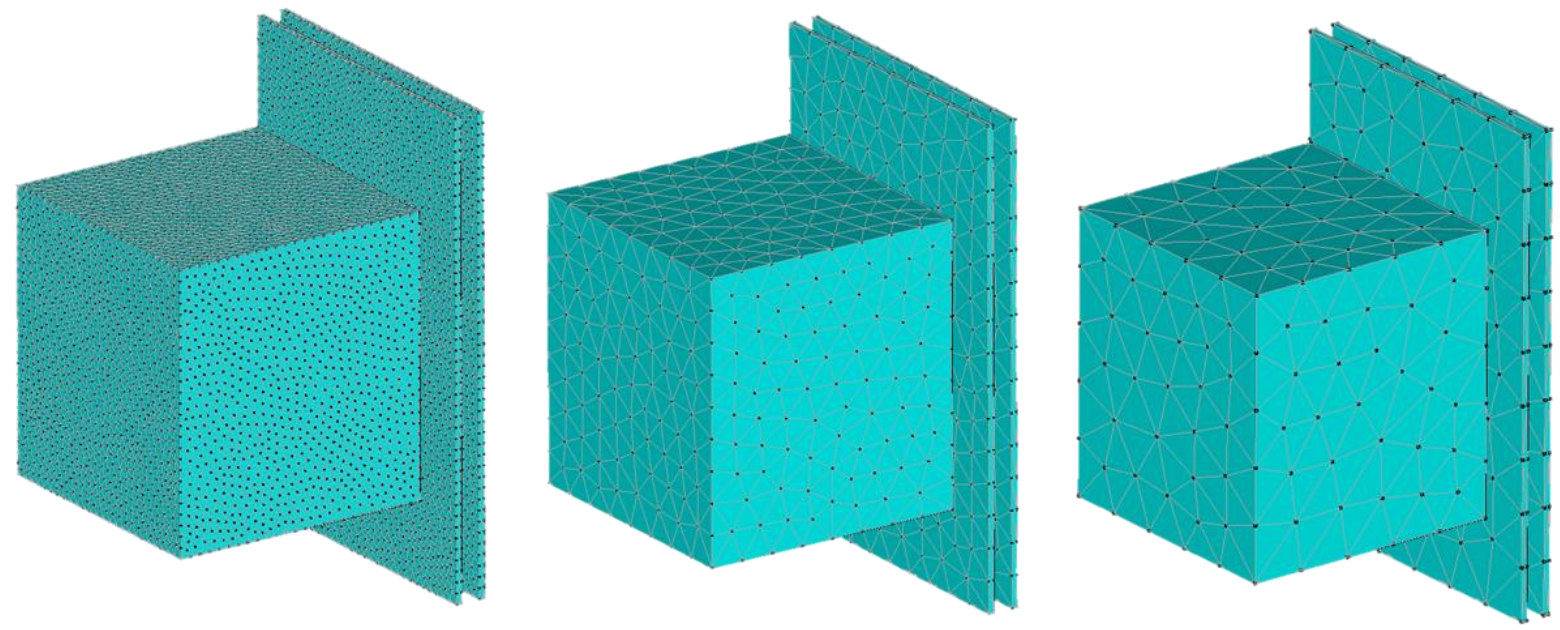

Figure 4-32. (a) 2" BE Mesh, (b) 6" BE Mesh, and (c) 10" BE Mesh 


\section{Conclusions}

With the emergence of new launch vehicles and the promise of continued human space exploration, there is a sustained need for accurate characterization of the acoustic environments in and around spacecraft inside the launch vehicle fairing. Spacecraft structures and sensitive instruments must be designed and integrated in a way that reduces their susceptibility to acoustic excitation. Improvements in analytical methods and computing power in recent years have resulted in better predictions for these acoustic environments and correlation with test data. At the advent of the space age in the 1960s, computers were not capable of the detailed simulations that are routinely performed today to model heat transfer, flight trajectories, and joint failure. Instead, detailed assessment of the capability of a spacecraft and its subassemblies was confined to comprehensive testing. As the space industry has matured and analytical predictions continue to demonstrate promising correlation with test data, there has been a gradual shift from solely testbased design evaluation to mixed test and analysis-based evaluation with analytical simulation increasingly relied upon in place of testing in some instances. For spacecraft constructed on heritage designs that have well-documented and consistent test results, small design changes can be evaluated analytically. This is advantageous, as a significant portion of the cost for any spacecraft comes from the testing process.

In this work, the two acoustic testing methods used to qualify spacecraft for flight, RFAT and DFAT, have been presented and their defining attributes have been compared. The fundamental characteristics of DFAT have been explored, with regards to the physical test configuration, properties of the acoustic field, and control methodology. The associated testing procedure is also described, consistent with industry standards, to describe how test data used in this work was recovered from an acoustic field shaping run using a spacecraft mockup. 
Next, the coupled FEM-BEM analysis method was detailed, starting with a survey of the development of BEM in the 1960s and 70s to solve fluid boundary problems, proceeding to a review of the fundamental theory employed in the coupled analysis, and concluding with a detailed description of the analysis procedure used for this work. A coupled analysis was performed for the acoustic model based on a modal test-correlated FEM of the spacecraft mockup. A microphone and an accelerometer were used to acquire data points for the acoustic environment between the spacecraft bus and closely-spaced solar array panels in the test and the corresponding pressure levels and structural responses were measured analytically for comparison.

In order to explore the capability of mixed test and analysis-based design evaluation, a trade study was conducted to observe the sensitivity of the predicted acoustic environment between structural panels to changes in the spacing between panels, damping assumptions, and BE mesh size.

\subsection{Summary of Results}

Using the baseline acoustic model, the coupled FEM-BEM analysis predicted the SPL measurements between the Bus and Solar Array panels and the corresponding structural response on the adjacent Solar Array panel with decent accuracy. Although there is a slight mismatch in the prediction of the energy distribution in terms of frequency content between the test measurements and the analytical predictions, the overall energy levels measured in GRMS for the structural responses and OASPL for the acoustic field levels agree closely. Additionally, the energy distribution for the panel responses and the acoustic field between panels is concentrated at similar frequencies, indicating that there is some amount of modal coupling between the acoustic cavities and the panels. 
Many of the observations from the trade study make intuitive sense. In the trades where the distances between adjacent panels were varied, it was observed that random vibration responses on the panel were most sensitive to changes in the volume of adjacent regions. For all trades, the solar array panel responses were highest at the center and corners, corresponding to points furthest from the solar array posts. Likewise, the SPL predictions in the field generated between panels were most sensitive to changes in the local volume size. The predicted SPL was highest at the center of each cavity and decreased in any direction radially outward from the center, converging to the ambient (input) field levels.

The assumption of a 4\% DLF across all analysis frequencies was shown to be acceptable in approximating the GRMS at the test accelerometer location; however, this assumption was based on a single test measurement. While this is an acceptable assumption for preliminary analyses of this type in standard practice, further test data points are necessary for improving this damping approximation and tailoring it to responses at specific frequencies.

Lastly, a mesh convergence study confirmed that the use of a 6" mesh size in the BEM solution is appropriate for making accurate analytical predictions in the chosen $20-400 \mathrm{~Hz}$ analysis range. While a 2" BEM mesh may accommodate analyses across a much larger bandwidth, the severely increased CPU time for the associated BEM solution yielded no discernable improvement in analytical predictions over the coarser 6" mesh. In addition, it was seen that reducing the mesh fidelity beyond the six elements per wavelength convention resulted in a significantly diverged solution.

In this work, it has been shown that the FEM-BEM analysis method utilizing a test-correlated spacecraft FEM and a relatively simple coupled acoustic BEM model can predict acoustic field levels and structural responses with sufficient accuracy at acceptable computational expense. 


\subsection{Future Work}

An unanswered question in this work is that if this DFAT was repeated for the same acoustic environment and the same test article multiple times, how would the acoustic field measurements at the point between panels vary from test to test? And how would the resultant average of subsequent test measurements on the $1 / 3^{\text {rd }}$ octave bands compare with the analytical predictions? The only way to answer this question would be to perform several additional tests with an increased number of measurement points both in the acoustic field and on the structure. It should be noted that this testing process requires extensive planning and resources are not available to repeat the test several times outside of proprietary needs. The analytical model presented here could be improved with correlation to more test data points from the acoustic tests; specifically, this would mean measuring the SPL with microphones at multiple points between panels for each of multiple test runs and instrumenting the adjacent panels with several working accelerometers to characterize the associated structural responses.

While the $4 \%$ DLF applied to all $1 / 42^{\text {nd }}$ octave bands is an acceptable assumption for the structural damping considered here, additional accelerometer measurements on the structure would be helpful for improving this damping approximation and tailoring the loss factor on different frequency bands. In the VA One solution, the same DLF must be applied to all FE Structures, so an alternative DLF spectrum would have to be valid across all structures of interest.

Another point for future work would be to find an improved method for analytically representing the direct acoustic field and structural excitation generated in a DFAT. Research efforts are underway to characterize this direct acoustic field environment and the results from this work could be used to develop an associated plane wave excitation in the commercial software. While the diffuse field assumption applied here for the environment between closely-spaced panels has 
been shown to be acceptable, analysis of exterior spacecraft surfaces and mounted components may merit alternative approaches.

Lastly, it would be interesting to measure the acoustic field generated around the dolly at the base of the spacecraft and characterize the influence of the floor and the dolly on the acoustic environments inside the spacecraft bus. For some spacecraft designs with large central cavities open at the base, this solid floor boundary condition combined with the fixed-base boundary condition of the steel dolly may cause large amounts of acoustic energy to be refracted up into the spacecraft. These same boundary conditions are not necessarily present in the launch vehicle fairing or the alternative reverberant chamber testing environment, so their influence on the structural response of the spacecraft should be considered. 


\section{Bibliography}

[1] H. Himelblau, J. Manning, D. Kern, and A. Piersol, NASA-HDBK-7005: Dynamic Environmental Criteria. 2001.

[2] Dept. of Defense, "MIL-STD-810G w/Change 1," 2014, p. 515.7-i-515.7B-4.

[3] K. M. Eldred, "NASA-SP-8072: Acoustic Loads Generated by the Propulsion System," pp. $3-5,1971$.

[4] NASA, NASA-HDBK-7008: Spacecraft Dynamic Environments Testing. 2014.

[5] P. Larkin, "Developments in Direct-Field Acoustic Testing," Sound Vib., vol. 11, no. 48, pp. 6-10, 2014.

[6] NASA, NASA-HDBK-7010: Direct Field Acoustic Testing (DFAT). 2016.

[7] A. R. Kolaini and M. O'Connell, "A Discussion on the Potential Acoustic Chamber / Structure Modal Coupling During Qualification Testing of Flight Hardware," in Spacecraft and Launch Vehicle Dynamics Environments Workshop, 2008.

[8] A. R. Kolaini, B. Doty, and Z. Chang, "Reverberant Acoustic Testing and Direct Field Acoustic Testing Acoustic Standing Waves and Their Impact on Structural Responses,” in Spacecraft and Launch Vehicle Dynamic Environments Workshop, 2012.

[9] J. W. Rouse, M. Mesh, and E. C. Stasiunas, "Analytical Modeling of the Acoustic Field during a Direct Field Acoustic Test," J. Institute of Environmental Sciences and Technology, vol. 54, no. 2, pp. 1-54, 2011.

[10] M. B. Van Dyke, "Test Observations of the Characteristics of the Acoustic Field Generated by High Intensity Loudspeaker Testing," in Spacecraft and Launch Vehicle Dynamic 
Environments Workshop, 2013.

[11] United Launch Alliance, Delta IV Launch Services User's Guide. 2013.

[12] United Launch Alliance, Atlas V Launch Services User's Guide. 2010.

[13] SpaceX, Falcon 9 Launch Vehicle Payload User's Guide. 2015.

[14] A. H. D. Cheng and D. T. Cheng, "Heritage and early history of the boundary element method," Eng. Anal. Bound. Elem., vol. 29, no. 3, pp. 268-302, 2005.

[15] M. B. Friedman and R. Shaw, "Diffraction of Pulses by Cylindrical Obstacles of Arbitrary Cross Section," J. Appl. Mech., vol. 29, no. 1, pp. 40-46, 1962.

[16] R. P. Banaugh and W. Goldsmith, "Diffraction of Steady Acoustic Waves by Surfaces of Arbitrary Shape," J. Acoust. Soc. Am., vol. 35, no. 10, pp. 206-209, 1963.

[17] L. H. Chen, "Sound Radiation from an Arbitrary Body," J. Acoust. Soc. Am., vol. 35, no. 10, p. 1626, 1963.

[18] L. G. Copley, "Integral Equation Method for Radiation from Vibrating Bodies," J. Acoust. Soc. Am., vol. 41, no. 4, p. 807-816, 1967.

[19] H. A. Schenck, "Improved Integral Formulation for Acoustic Radiation Problems," J. Acoust. Soc. Am., vol. 44, no. 1, pp. 41-58, 1968.

[20] W. Benthien and a. Schenck, "Nonexistence and nonuniqueness problems associated with integral equation methods in acoustics," Comput. Struct., vol. 65, no. 3, pp. 295-305, 1997.

[21] A. J. Pretlove, "Free Vibrations of a Rectangular Panel Backed by a Closed Rectangular Cavity," J. Sound Vib., vol. 2, no. 3, pp. 197-209, 1964.

[22] A. J. Pretlove, "Forced Vibrations of a Rectangular Panel Backed by a Closed rectangular 
Cavity,” J. Sound Vib., vol. 3, no. 3, pp. 252-261, 1966.

[23] S. Kopuz, Y. S. Unlusoy, and M. Caliskan, "Integrated FEM BEM approach to the dynamic and acoustic analysis of plate structures.pdf," Eng. Anal. Bound. Elem., vol. 17, pp. 269$277,1996$.

[24] W. Ben Tsoi, A. R. Kolaini, and B. W. Childs, "Vibration on Composite Reflector: Test versus Prediction," in Spacecraft and Launch Vehicle Dynamic Environments Workshop, 2008, pp. 1-32.

[25] F. Ruess, H. Segelke, A. Obst, and A. Rubio, “Acoustic Test Prediction and Correlation for the SGEO Satellite Platform STM Model," in 13th European Conf. on Spacecraft Structures, Materials \& Environmental Testing, 2014, no. April, pp. 1-4.

[26] B. Gardner, A. Medeiros, L. Alimonti, A. Castel, and C. Musser, "Investigating DFAT Diffusivity Using Wavenumber- Frequency Analysis with Boundary Element Models,” in Spacecraft and Launch Vehicle Dynamic Environments Workshop, 2017.

[27] D. Inoyama, R. Agarwal, and T. Stoumbos, "Prediction of Internal Cavity Vibro-Acoustic Environment for Space Vehicles," 55th AIAA/ASME/ASCE/AHS/ASC Struct. Struct. Dyn. Mater. Conf., no. January, pp. 1-7, 2014.

[28] D. Inoyama, R. Agarwal, and T. Stoumbos, "Analytical Prediction and Test Correlation of Spacecraft Cavity Acoustic Environment," 56th AIAA/ASCE/AHS/ASC Struct. Struct. Dyn. Mater. Conf., no. January, pp. 1-15, 2015.

[29] D. Inoyama, R. Agarwal, and T. Stoumbos, “Acoustic analysis of a partially open spacecraft cavity using multi-domain boundary element method," 57th AIAA/ASCE/AHS/ASC Struct. Struct. Dyn. Mater. Conf. 2016, no. January, pp. 1-19, 2016. 
[30] H. Djojodihardjo, "Vibro-acoustic analysis of the acoustic-structure interaction of flexible structure due to acoustic excitation,” Acta Astronaut., vol. 108, pp. 129-145, 2015.

[31] F. Holström, "Structure-acoustic analysis using BEM/FEM; Implementation in Matlab," 2001.

[32] VA One, SEA and BEM Vibro-Acoustic Analysis, Software Package, Ver. 2016.1, ESI Group, Paris, France, 2016 


\section{Appendix A: Acoustic Analysis in VA One}

Analytical tools for simulating acoustic environments and the transmission of sound energy between volumes and structures utilizing BEM and SEA techniques are relatively new compared to those purely concerned with structural dynamics utilizing FEM. For this reason, some of the assumptions specific to the VA One software [32] in BEM-FEM coupling and analysis are explained as they relate to the theoretical background presented in Chapter 3.

\section{A.1 Coupled FEM-BEM Modeling Process}

A visual overview of the FEM-BEM modeling processes is presented in Figure A-1. The acoustic model in VA One is created by first importing portions of the FEM to be considered for acoustic excitation. As stated previously, only certain NASTRAN element types are recognized by VA One and many aspects of the FEM are not necessary to create the model and perform the analysis. In this case, a .DAT file containing FE meshes from the spacecraft FEM consisting of PCOMP and PSHELL elements was imported into the model. VA One allows the user to convert the desired FE mesh into FE Structural Subsystems that retain the elastic structural modes. Modal analysis is conducted on the FEM in NASTRAN separately and the .OP2 results file containing the modal eigenvalues and eigenvectors is imported into VA One and linked with the FE Structure; this represents the intermediate finite element solution for the structure. The software retains the node IDs from the FEM for any imported FE Structure, so it only reads the relevant modal data for the existing FE Structure. This means that for a large .OP2 file, VA One will only import the displacement results for nodes existing in the acoustic model. A consistent DLF spectrum equivalent to twice the damping ratio is assigned to all FE structures in the model.

In addition to the creation of FE Structural Subsystems, the software generates coincident FE Faces consisting of triangular shell elements to represent the fluid boundary surfaces for the intermediate 
BEM solution. These FE Faces can take on one of two boundary conditions: either the face is considered "Elastic", in which case it is coupled to the normal mode shapes of the underlying FE Structure, or the face is considered "Rigid", and decoupled from any structural modes so that the zero velocity Neumann boundary condition is specified for every node on that surface. Each FE Face has a "Front" and "Back" surface that can receive plane wave excitation. In the case of the box enclosed by six surfaces presented here, it would be possible to consider some exterior acoustic field and a distinct interior acoustic field and create multiple domains, but for this work only the exterior acoustic environment is modeled and the outside "Front" surfaces of the FE Faces are the only ones subject to excitation. In VA One, the acoustic field for a given BE domain is defined by a BEM Fluid, which supplies the equivalent acoustic excitation generated by a system of plane waves to the selected surfaces of the connected FE Faces.
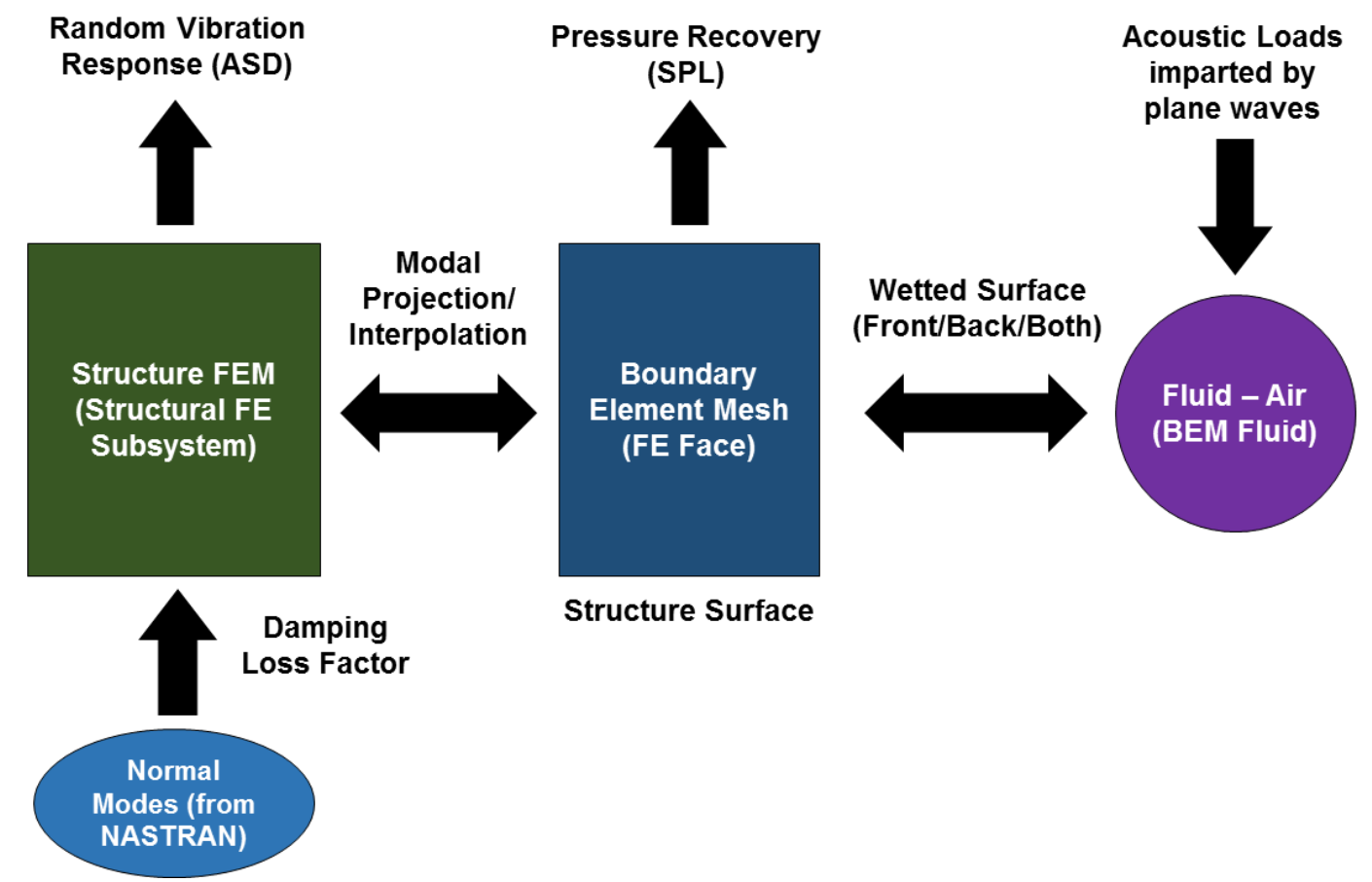

Figure A-1. VA Coupled FEM-BEM Model Schematic

In the acoustic model presented in this work, the exterior acoustic domain is represented by a single BEM Fluid that supplies the same excitation to the exterior of all FE Faces in the model as depicted 
in Figure A-2, where the cloud of 50 plane waves distributed around the BEM fluid represents the diffuse field assumption.

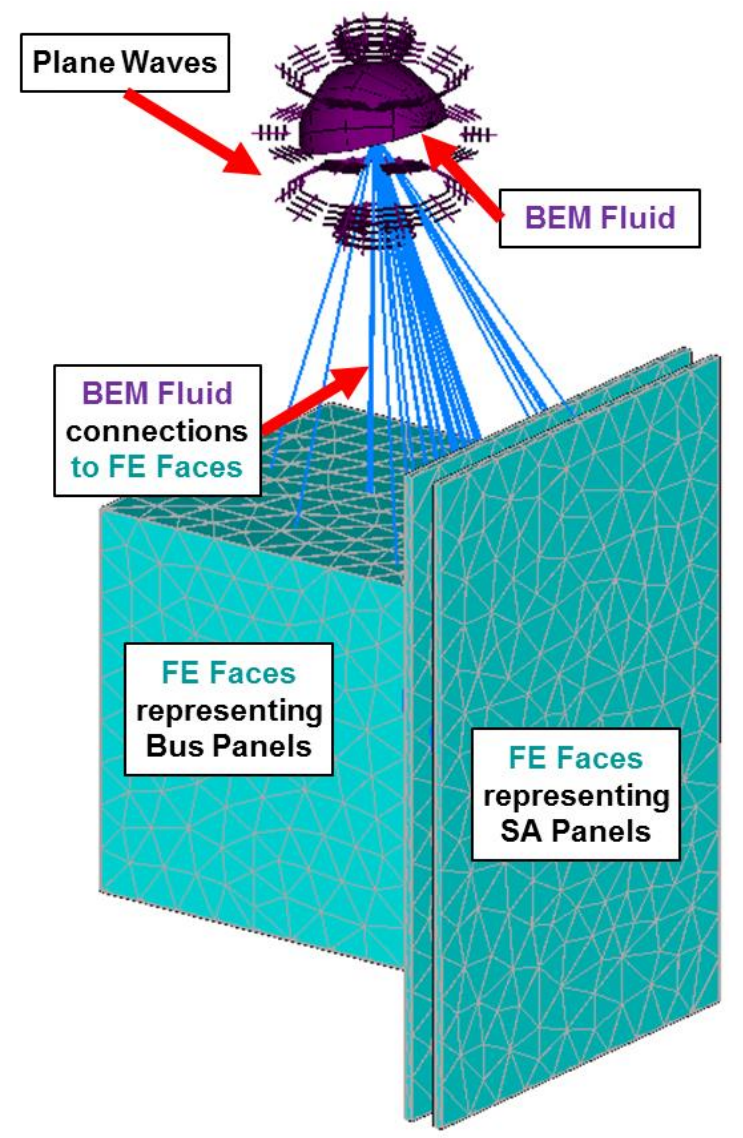

Figure A-2. VA One Model with BEM Fluid Connection Shown

\section{A.2 VA One Model Solution}

With both the FE structural and BE acoustic domains correctly defined and coupled, the intermediate BEM solution is performed for the acoustic domain over a series of frequency bands for the desired bandwidth specified in fractions of an octave $\left(1 / 3^{\text {rd }}, 1 / 12^{\text {th }}, 1 / 42^{\text {nd }}\right.$, etc. $)$ using the RAYON solver. Depending on the nodal density of the BE surfaces and the number of plane waves considered for the excitation, this intermediate BEM solution can be the most expensive part of the overall acoustic analysis in terms of CPU time. 
Once the intermediate BEM solution is complete, the final coupled acoustic solution for the structure and fluid domains is performed. Using the same frequency bandwidth specified for the intermediate BEM solution, the final solution interpolates between the nodal displacements from the FE structure at each of the structural modes and the nodal velocities and pressures from the acoustic boundary surfaces on each $1 / n^{\text {th }}$ octave frequency band. The CPU time for the coupled system solution will vary based on the number of structural modes considered, the frequency range of interest, and the amount of results data requested.

\section{A.3 Data Recovery}

Results are obtained for the structure and acoustic field at multiple points using sensors placed on nodes or elements because limiting the requested results to specific locations of interest can significantly reduce CPU time. In the structural domain, the stress on particular elements and contour over surfaces can be recovered in addition to the nodal forces and displacements. For the analysis in this work, nodal accelerations corresponding to points of interest on the solar array panels were acquired using displacement sensors in order to measure the random vibration response in terms of power spectral density for direct comparison with accelerometer data. Likewise, nodal velocities and pressures can be recovered at discrete points in the fluid domain. In order to recover the nodal pressures corresponding to microphone locations in the acoustic field, a single triangular data recovery face is created with a pressure sensor attached to one of the three nodes. This sensor acquires the sound pressure levels at that field point for direct comparison with the test microphone data. 